INTERSTITIAL LASER COAGULATION FOR HEPATIC TUMOURS 
CIP gegevens

ISBN 90-73235-38-3

(C) $2000 \mathrm{~J}$. Heisterkamp. All rights reserved. No part of this thesis may be reproduced, stored in a retrieval system of any nature, or transmitted in any form by any means, electronic, mechanical, photocopying, recording or otherwise, without the permission of the author

Printed by Optima Grafische Communicatie, Rotterdam, The Netherlands 


\title{
INTERSTITIAL LASER COAGULATION FOR HEPATIC TUMOURS
}

\author{
INTERSTITIËLE LASER COAGULATIE \\ VOOR LEVER TUMOREN
}

\section{PROEFSCHRIFT}

Ter verkrijging van de graad van doctor aan de Erasmus Universiteit Rotterdam op gezag van de Rector Magnificus

Prof. dr P.W.C. Akkermans M.A

en volgens besluit van het College voor Promoties

De openbare verdediging zal plaatsvinden op woensdag 2 februari 2000 om 15.45 uur.

door

Joos Heisterkamp

geboren te Nijmegen 


\title{
Promotie commissie
}

\author{
Promotor: $\quad$ Prof. dr J. Jeekel \\ Overige leden: $\quad$ Prof. dr. G.P. Krestin \\ Prof. dr. G. Stoter \\ Prof, dr. O.T. Terpstra \\ Co-promotores: $\quad$ Dr. J.N.M. IJzermans \\ Dr. R. van Hillegerberg
}

The research presented in this thesis has been performed at the Department of Surgery of the Erasmus University Rotterdam and University Hospital Rotterdam, The Netherlands. The Departments of Radiology, Pathology and Epidemiology and Biostatistics of the Erasmus University Rotterdam and the Department of Clinical Science MR, Philips Medical Systems, Best, The Netherlands, participated.

The research was financially supported by the University Hospital Rotterdam with a Revolving Fund and the Stichting Gastrostart.

The publication of this thesis was financially supported by: Johan Vermeij Stichting, Baxter Hyland Immuno Division, B. Braun Medical, B.L.W. Visser, CardioFocus, Janssen-Cilag, Luxtron, Schering, Somatex, Stöpler, Tatttoo Bob Pleinweg: "de enige echte" en Zambon. 


\section{Preface}

It is generally accepted that surgical resection is the therapy of choice for colorectal liver metastases and primary liver cancer and should be performed whenever technically and medically feasible. However, the vast majority of hepatic tumours is irresectable, due to number, distribution and/or location. It is estimated that only $20-35 \%$ of the patients with tumours confined to the liver are eligible for surgery. The rate of irresectability is even higher when a repeated resection is considered after recurrence in the liver following a previous liver resection. Therefore, it is mandatory to develop novel strategies to obtain local tumour control in the liver. An array of new approaches are currently being investigated. A definitive treatment modality has not yet been established.

In '82 interstitial laser coagulation (ILC) was introduced as a method aiming at local hepatic tumour destruction. In ' 88 the first investigations to the use of laser in destroying hepatic tumours were performed in Rotterdam at the Laboratory for Experimental Surgery of the Erasmus University Rotterdam. These initial studies led in '90 to the research program Laser treatment of experimental liver metastases. The studies described in this thesis are a continuation. In the General Introduction the current state of Interstitial laser coagulation for hepatic tumours is described and the aims of the studies are defined. The second part contains studies to the increase in the volume of coagulation. In the third part aspects of monitoring the laser-tissue interaction are investigated. In the final part general considerations are given for clinical application. 



\section{Table of contents}

\section{Part I General introduction}

1 Interstitial laser coagulation for hepatic tumours: An overview Adapted from: British Journal of Surgery, 1999: 86(3);293-304

2 Scope, aims and outline of the thesis

\section{Part II Increasing lesion size}

3 Heat resistant cylindrical diffuser for Interstitial laser coagulation: Comparison with the bare-tip fiber in ex vivo porcine liver

Lasers in Surgery and Medicine, 1997: 20 (4);304-309

4 Interstitial laser coagulation with four cylindrical-diffusing fibre tips: Importance of mutual fibre distance Lasers in Medical Science, 1999: 14(4);216-220

5 The importance of eliminating portal flow in producing large lesions with Interstitial laser coagulation in vivo British Journal of Surgery, 1997: 84(9);1245-1248

\section{Part III Laser-tissue interaction and monitoring}

6 Long-term effects of Interstitial laser coagulation in porcine liver with portal inflow occlusion: Central versus peripheral lesions Jotmal of Vascular and Intersentional Radiology, 1999, 10(6);825-831 
7 NADH activity and DNA integrity in human hepatic metastases after Interstial laser coagulation

Submitted for publication

8 Critical temperature and heating time for coagulation damage: Implications for interstitial laser coagulation of tumours Lasers in Surgery and Medicine, 1999: 25(3);257-262

9 Accuracy of MR-phase mapping for temperature monitoring during Interstitial laser coagulation in the liver: At rest versus simulated respiration in 2 ex vivo models and in vivo Adapted from: Magnetic Resonance in Medicine, 1999: 41(5);919-925

\section{Part IV General discussion}

10 Discussion and considerations for clinical applications

11 Conclusions

\section{Appendices}

Bibliography

Summary

Samenvatting voor de leek

Contributing authors

Acknowledgments

Curriculum vitae auctoris 



\section{Interstitial laser coagulation for hepatic tumours: An overview}

Adapted from: Interstitial laser coagulation for hepatic tumours. J Heisterkamp, $\mathrm{R}$ van Hillegersberg \& JNM IJzermans. British Journal of Surgery 1999, 86; 293-304

\subsection{INTRODUCTION}

Partial liver resection is the sole therapy giving a chance of cure for patients with colorectal metastases (Steele, Jr. et al, 1991; Scheele et al, 1995; Nordlinger et al, 1996) and patients with hepatocellular carcinoma (HCC) (Livraghi et al, 1997).

Cancer of the colon and rectum is second to lung cancer in incidence of malignant disease in both men and women. Currently it accounts for 9500 new cases per annum in the Netherlands (Centraal Bureau voor de Statistiek, 1998). At time of initial presentation, hepatic metastases are present in $25 \%$ of patients and in an additional $45 \%$, hepatic metastases will be diagnosed in follow up after primary tumour resection (King's Fund Forum, 1990). Resection results in a five-year survival rate of $25 \%-39 \%$.

$\mathrm{HCC}$ is a common malignancy in oriental countries and Africa with an increasing incidence in the Western world (El-Serag and Mason, 1999). In the last 10 years activation of screening programs has resulted in a relative increase in the number of resectable cases (IJzermans and Bac, 1997; Izzo et al, 1998). For hepatocellular carcinoma 5-year survival following partial hepatectomy is $12 \%$ to $39 \%$, also depending on the underlying liver disease.

Liver resections are now performed with acceptable morbidity and minimal mortality (Nordlinger $e t$ $a l, 1996)$, but resection is applicable in only a fraction of the patients with primary and secondary hepatic tumours. More than $80 \%$ of the patients with hepatic metastases of colorectal carcinoma do not fulfil the criteria that justify partial hepatectomy (Wanebo et al, 1996; Herfarth and Kallinowski, 1997). Resection of HCC in cirthotic liver is even further restricted by the high operative risk due to the poor general status of the patients and/or the reduced functional hepatic capacity. 
Alternative therapies have been developed, aiming at local, regional or systemic treatment (reviewed by Vahrmeijer et al, 1995 and Cromheecke et al, 1999). Such a local modality: Interstitial laser coagulation (ILC), introduced in ' 82 (Bown, 1983), has matured in recent years. ILC is a thermal technique aiming at coagulation of solid tumours in parenchymal organs by local light delivery using thin flexible fibre(s) (Figure 1.1). In implanted tumour models it was found that ILC may lead to complete coagulation with a complete tumour response (Matthewson et al, 1989). Rapidly clinical case-series were available (Masters et al, 1991; Steger et al, 1992a). Whilst the ILC procedure itself was judged feasible and tolerable, optimisation of the technique was mandatory for successful application in tumours larger than approximately $2.5 \mathrm{~cm}$ in diameter. Solutions to the problems and questions, raised in these studies were reinvestigated in preclinical models and several improvements have already been evaluated in patients. In this chapter the background of ILC, its development and current state are described with reference to physical, histological and radiological aspects.

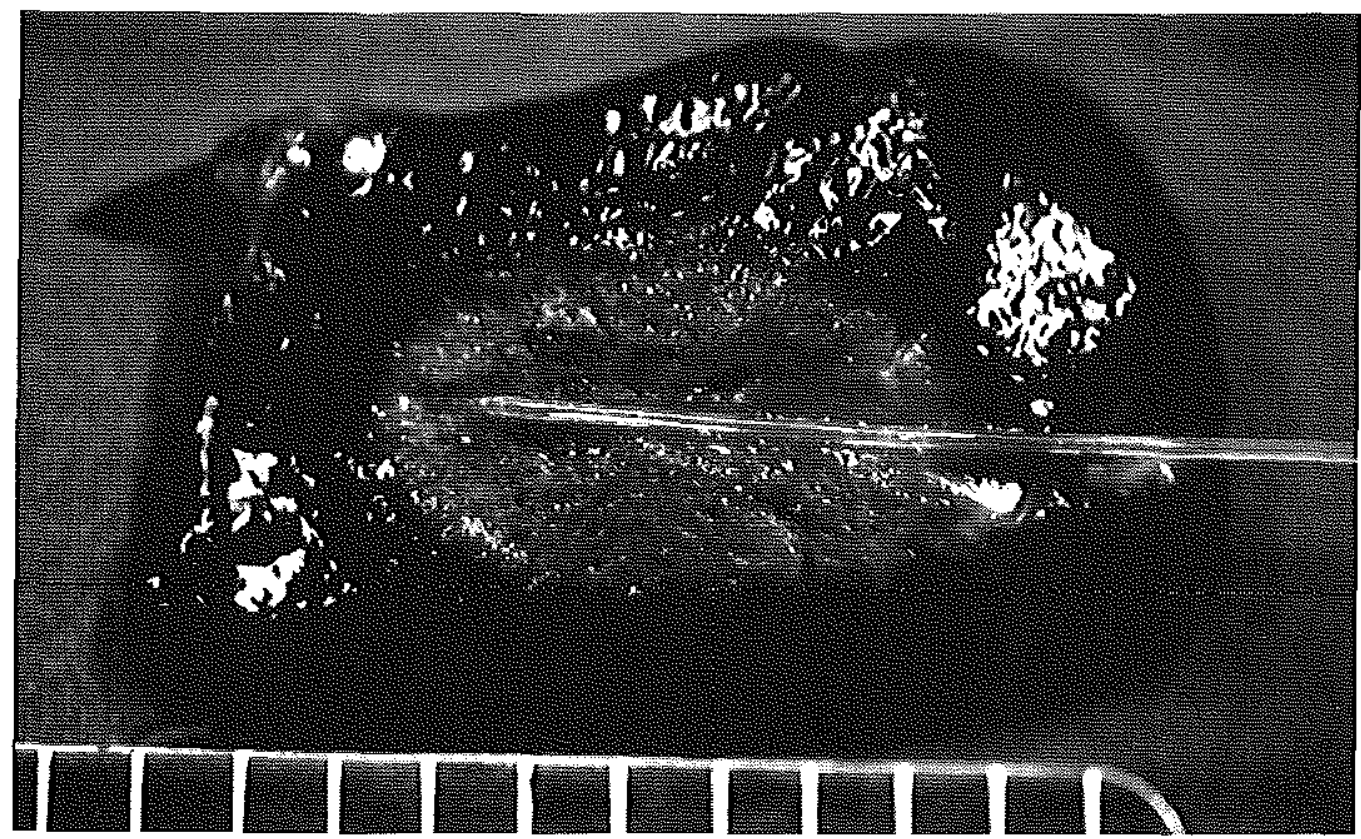

Figure 1.1 Macroscopic view of a typical coagulated lesion produced in ex vivo porcine liver. After laser treatment (6W during 6 minutes of Nd:YAG light, 2160 Joules) the liver was sectioned in the plane along the laser fibre which is in situ (1.65 mm in diameter with a cylindrical diffusing tip of $2 \mathrm{~cm})$. The whitish area is the resulting area of coagulation; (scale in $5 \mathrm{~mm}$, largest length: $36 \mathrm{~mm}$ and diameter: 26 $\mathrm{mm}$ ). 


\subsection{LOCAL TREATMENT}

\section{Rationale}

As segmental and even sub-segmental liver resections yield results akin larger partial hepatectomies (Nagasue et al, 1985; Nordlinger et al, 1996; Rees et al, 1997), provided the resection margin is free of tumour cells (Yamamoto et al, 1999), there is a rational for local tumour destruction. When complete fumour destruction can be achieved by a local treatment modality, survival rates may be comparable to surgery. Moreover on theoretical grounds, stimulation of dormant malignant cells may be less when using local approaches (Gutman and Fidler, 1995) as growth stimulating effects on tumour, induced by hepatic damage and regeneration, have been demonstrated (Slooter et al, 1995).

\section{Comparable local treatment modalities}

Despite substantial research efforts, no definitive local treatment modality has yet been established. In general the various types of assaults (either by cold, heat or dehydration) lead to instant cell death. With cryosurgery the largest clinical experience is available (Lee et al, 1997). Through hollow probes ( 5 millimetres to 1 centimetre in diameter) liquid gas is pumped with evaporation at the tip, resulting in very low temperatures $\left(-180^{\circ} \mathrm{C}\right)$ with a temperature gradient in the tissue. Results are variable: ranging from complete responses in a majority of cases (Ravikumar et al, 1991a; Weaver et al, 1995; Crews et al, 1997) to a recurrence rate of 50\% (Ravikumar et al, 1994; Adam et al, 1997; Johnson et al, 1997). Because of the need for a laparotomy due to the large probe size, cryosurgery is impractical for repeated treatment, although laparoscopic approaches are being investigated (Cuschieri et al, 1995; McCall et al, 1996; Ravikumar; 1996).

Percutaneous alcohol injection, effective in obtaining growth arrest for primary liver cancer (Bartolozzi and Lencioni, 1996; Di Stasi et al, 1997), is less suited for secondary hepatic tumours (Livraghi et al, 1993; Giovannini and Seitz, 1994). Complete tumour destruction is hampered by uneven distribution of the liquid within the firm tumour and irregular spread into the softer liver.

Radiofrequency (RF) coagulation can also be performed percutaneously and requires the insertion of rigid probes (several millimetres in diameter) in the tumour (Rossi et al, 1996; Solbiati et al, 1997). Electric currents in the tissue result in a temperature increase with a cytotoxic effect comparable to laser coagulation (Patterson et $a l, 1998$ ). RF is inexpensive but incomplete tissue destruction has been described as a result of the variable electrical conductivity within tissue (Scudamore et al, 1997; Trondsen et al, 1998). In a single centre-experience a newly developed 
catheter resulted in a remarkable improvement in the number of completely coagulated tumours in patients (Curley et al, 1999). Confirmation of these results in other series is awaited.

Focused ultrasound (FUS) represents the ultimate minimal invasive modality. In extracorporal arrays of radio-frequency generators, electric waves are emitted converging at a focus where a maximal synergistic effect is obtained (Sibille et al, 1993; Hill and ter Haar, 1995). Accumulation outside the focus does not occur and thus no thermal effect is built up in the dermal layers and other structures in the course to the target. The volume of the focus (and thus the maximal volume of coagulation per shot) is $4-8 \mathrm{~mm}^{2}$, thus placement and control of this the focus demands high quality of imaging techniques. As both FUS and the imaging systems are not yet precise enough, FUS does not qualify for clinical application (Yang et al, 1993; Jolesz and Blumenfeld, 1994).

The techniques, described above, like $\Pi \mathrm{LC}$ have their own specific pros and cons. Currently none of these local modalities have been proved effective and therefore should be used only as a component of a clinical study (Consensus Statement, 1997).

\subsection{HISTORY OF ILC}

The first reported interstitial laser treatment was performed in ' 82 by Bown in London in a patient with a skin metastasis of bronchus carcinoma in which a single fibre was introduced for delivery of high power (20W) Nd:YAG light for a short duration of 10 seconds (1983). Indeed, localised tumour necrosis was found on the place of the fibre tip, although still surrounded by viable tumour cells. At the same time, Ascher in Graz coagulated interstitially a meningeal tumour, judged inoperable at craniotomy, by placing a laser scalpel in the tumour with lower laser power than usually for cutting [personal communication 1996]. Despite early paralysis as a of swelling of the coagulated volume, the patient rapidly recovered.

Several names have been used for this treatment: Bown, as pioneer, baptised the technique interstitial laser hyperthermia (Masters and Bown, 1990). To prevent confusion with traditional hyperthermia (with lower temperatures) this was changed subsequently in interstitial laser phototherapy (ILP) (Amin et al, 1993d) pronouncing the use of light, both in laser and in photo. Synonyms, such as laser induced thermotherapy (LITT) (Vogl et al, 1997), interstitial thermotherapy (ILT) (Tranberg et al, 1996) and interstitial laser ablation (ILA) (Nolsoe et al, 1989) have been introduced as well. The latter, however is inaccurate for the tissue effect, as ablation and coagulation are discrete thermal events. It would be convenient if a single term could be agreed 
upon. The herein-proposed interstitial laser coagulation (ILC) fulfils all criteria by unambiguously describing the anatomical position of the treatment (interstitial), the means of the treatment (laser) and the effect of the treatment (coagulation).

\subsection{LASER TISSUE INTERACTION}

In ILC laser light at low powers (3-15 Watts) is used with long exposure times (3-20 min) resulting in coagulation, visible as blanching of the tissue, as in a boiled egg (Figure 1.1). The treated area is left to undergo necrosis in situ with subsequent healing by regeneration and/or fibrosis. Selection of tissue destruction is a function of both fibre position(s) within the tumour and the temperature gradients created by the amount of power and duration of exposure. The subsequent biological response to laser light depends upon the interaction of the light with the tissue.

\section{Optical aspects}

Laser light has three unique properties enabling reliable and direct transmission of high amounts of energy over long distances: 1) waves of same wavelength (monochromatic), 2) waves in phase with each other (coherent) and 3) waves parallel to each other (collimated); reviewed by van Hillegersberg (1997).

As the wavelength of the used laser light increases, penetration of light through tissue decreases. The compromise between deep penetration depth and sufficient absorption in the tissue determines the optimal wavelength for $\mathrm{LC}$. Nd:YAG (wavelength of $1064 \mathrm{~nm}$ ) and Diode light $(890 \mathrm{~nm}$ ) have been investigated (Amin et al, 1993b; Mesecke von Rheinbaben et al, 1996; Prapavat et al, 1996). Both wavelengths are minimally absorbed by water but merely by tissue chromophores. Nd:YAG has a deeper penetration depth: up to $10 \mathrm{~mm}$ (van Hillegersberg, 1997).

As tumour and liver differ in colour, the optical characteristic might differ as well. Van Hillegersberg et al. (1993b) found no net difference in absorption coefficient between rat tumour and liver at $1064 \mathrm{~nm}$; scattering was only slightly higher in tumour. More important are changes in optical characteristics in the process of coagulation (during laser treatment). Van Hillegersberg et al. (1994) measured light during laser treatment of a tumour implanted in rat liver. Light uptake, in a probe peripheral from the laser fibre, increased during treatment suggesting that absorption in the coagulated area decreased and light penetration increased. Recently this has been confirmed by determination of the optical characteristics of vital and coagulated tissue (Ritz et al, 1997). The 
continuous flow of light to already coagulated tissue might result in an excessive temperature rise with carbonisation and decreased light penetration.

Eventually, lesion size is limited at a plateau due to decrease of light density and conducted heat at the tissue peripheral from the laser fibre (Svaasand et al, 1990). Both power of the light (Watts) and duration of exposure ( $\mathrm{min}$ ) may be varied to set total energy (Joules).

\section{Thermal aspects}

Upon absorption of the laser light several thermal effects occur (Table 1.1). Coagulation is defined as the irreversible thermal damage of tissue proteins at temperatures between $55^{\circ} \mathrm{C}$ and $95^{\circ} \mathrm{C}$. Formation and the extent of coagulation are dependent on the time during which the temperature remains at this range. Although it is generally assumed that in tissue warmed at $60^{\circ} \mathrm{C}$ for several minutes complete protein denaturation occurs, experimental data are lacking. If the temperature exceeds $100^{\circ} \mathrm{C}$, water vaporisation and tissue ablation occur (van Hillegersberg, 1997). These effects are used with laser as a surgical tool for cutting and for haemostasis. It is important to consider that temperatures far from the laser tip are determined by heat conduction from the site of light absorption (Svaasand et al, 1990), rather than by direct transmission of light.

Table 1.1 Tissue temperature and tissue effects

\begin{tabular}{cll}
\hline Temperature $\left({ }^{\circ} \mathrm{C}\right)$ & Physical effect & Biological effect \\
\hline $40-45$ & & Enzyme inactivation \\
$60-140$ & Protein denaturation & Cell shrinkage, hyperchromasia, membrane \\
& & rupture, pyknosis and hyalinisation of collagen \\
$100-300$ & Water vaporisation & Extracellular stean vacuole and cell shrinkage \\
$300-1000$ & Carbonisation and Ablation & Ablation, vaporisation and carbonisation \\
\hline
\end{tabular}

After: Thomsen (1991), Hunter and Dixon (1985) and Welch et al. (1991). Adapted from van Hillegersberg (1993). 


\section{Interstitial laser fibres}

The research to the optimal tip of the laser fibre has been determined by concerns regarding carbonisation of tissue surrounding the fibre tip. Carbonisation occurs at temperatures above $300^{\circ} \mathrm{C}$ (Table 1.1) and this blackish tissue limits the optical penetration into the surrounding tissue with further light and thus temperature accumulation (Welch et al, 1991). In Figure 3.1 the two laser fibres currently used in ILC are shown. Pilot light (red) is emitted which reveals the specific light emission pattern.

This process of carbonisation occurs with the use of a bare-tip fibre where light emission is concentrated at the tip. The fundamental process of distributing the light into the tissue is lost. As a consequence, the temperature profile from a carbonised tip is not different than from any other type of hot tip; one might as well use a hot knitting needle. With so-called cylindrical diffusing fibre-tips a homogeneous light emission is produced over its light diffusing length. By that, the emitted laser light is equally distributed into the tissue, and the induced coagulation depends on light transmission followed by heat production upon absorption in the tissue (Svaasand et al, 1990). However, until recently the available cylindrical diffusing fibre tips were too large in diameter and temperature unstable.

As eventually coagulation size produced with a single fibre is limited, simultaneous multiple fibre application has been proposed for lesions $>2 \mathrm{~cm}$ in diameter. Synergism between multiple fibres was shown in a mathematical model (Davis et al, 1989). A four to six fold increase in lesion volume by the synergistic effect of simultaneous multiple fibre application was predicted. Both a reduced heat dissipation in the centre of the fibres and an additive effect at the borders of the individual lesions account for this effect (Svaasand et al, 1985; Wyman, 1993). To apply multiple fibres simultaneously, several beamsplitting devices have been developed. Although beamsplitters are no novelty in applied optical physics, their use in $\Pi \mathrm{LC}$ has been limited to several prototypes (Frank and Hessel, 1990; Steger et al, 1992a; Bosman, 1993; 17). The described beamsplitters can not withstand laser output powers higher than $10 \mathrm{~W}$ due to power loss with subsequent overheating.

\section{Histopathological evidence of effects}

Tissue whitening is associated with global thermal denaturation but is not a good indicator of the exact outer boundary of the lethal tissue effect. Histopathological studies showed that the borders of more peripheral zones of thermal damage contain haemorrhage, hyperaemia and oedema. This haemorraghic zone is a typical finding after laser coagulation in a variety of tissues and is explained 
by thermal intravascular erythrolysis (Thomsen, 1995).

Notwithstanding the difficulties in defining coagulation damage, a rough outline can be given. In essence, no difference in reaction between tumour and normal tissue has been found. The acute interstitial laser lesion has a characteristic zonal architecture as described in detail in literature (Schober et al, 1993; Tracz et al, 1993; van Hillegersberg et al, 1994). Briefly, in a central zone surrounding the insertion of the laser probe the gross tissue structure is well preserved, whereas cellular build up is characterised by acidophilic cytoplasm and elongated nuclei. This zone is surrounded by a broad area, containing dilated sinusoids and cells with vacuolated cytoplasm. The outer, macroscopically detectable, haemonhaged zone consists of cells with less vacuolated cytoplasm and hyperaemic sinusoids.

In the process of regeneration a fibrotic rim is formed, delineating normal tissue from the coagulum and the coagulated tissue can be palpated as a dense mass. Regeneration takes place as mesenchymal reorganisation with proliferation of fibroblasts, endothelium and bile ducts (Steger et al, 1992a; van Hillegersberg et al, 1994; Isbert ef al, 1997). Typically the original architecture remains infact with no ingrowth of blood vessels inside the fibrotic rim. Incidentally abscess formation and central deliquesced necrosis have been found. Depending on initial size, complete regeneration of hepatic tissue takes place between 3 and 12 months post laser-treatment as demonstrated in normal liver of rabbits (Isbert $e t a l, 1997$ ) and dogs (Steger $e$ al, 1992a).

Whereas in normal liver tissue the area of coagulation can be estimated as the whitened tissue, this is not possible in already pale tumour. Morphological observations at microscopy do not allow unambiguous conclusions regarding the cell vitality directly post laser treatment. Cells with a nucleus have been described in H\&E stained biopsy specimens following ILC, that were found avital when these cells were studied with electron microscopy (Nolsoe et al, 1989). This has hampered studies to the effect of ILC in human hepatic tumours. Although providing indirect evidence, immuno-histochemical methods for enzymatic activity may represent the only indicators for thermal denaturation.

\subsection{STUDIES IN EXPERIMENTAL ANIMAL MODELS}

Studies in animal models to the value of $\Pi \mathrm{LC}$ have been performed in tumour bearing rodents (van Hillegersberg et al, 1994; Moller et al, 1997b) and rabbits (Hoye et al, 1968; Prudhomme et al, 1996) as well as in normal livers of rodents (Matsumoto et al, 1992; Amin et al, 1993e), canines (Steger et al, 1992a) and porcines (Dachman et al, 1990; Bosman et al, 1991; Malone et al, 1994; 
Germer et al, 1997; Moller et al, 1997a). In the tumour models the cytotoxicity of ILC was studied and in the normal-liver models strategies to increase coagulation volume could be studied at human scale.

Earliest documented work was by Matthewson et al. (1987) in rat liver using Nd:YAG light. A 0.4mm diameter fibre was inserted into the centre of a lobe of liver, exposed at laparotomy. Power settings of $0.5-2 \mathrm{~W}$ and exposure times of 50-2400 sec were used to give well-defined lesions of coagulative necrosis with sharp boundaries between normal and coagulated liver. The size of coagulation increased with increasing laser energy until a plateau was reached. The typical histological changes were found at microscopy. Van Hillegersberg et al. (1994) studied ILC in a rat liver tumour model. Lesion size and liver enzymes increased with laser energy applied. Deterioration in liver function was not found. At optimal laser settings complete tumour response was obtained. In addition, several models with subcutaneous implanted tumours in rodents showed the evident tumour-cell killing potential of ILC (Matthewson et al, 1989; Dowlatshahi et al, 1992a). In a second phase of animal experiments, modifications to enlarge the volume of coagulation were studied in large-animal models. Steger et al. (1992a) evaluated in dogs the use of a laser beamsplitting device to feed 4 fibres at a mutual distance of $1.5 \mathrm{~cm}$ which resulted in lesions of maximally $3.5 \mathrm{~cm}$.

Computer simulations (Roggan and Muller, 1994) and mathematical modelling (Whelan et al, 1995) predict a four- to five- fold increase of the area of coagulation in liver without blood flow. However, the relatively small size of previous animal models and the small volume of coagulation that could be produced may have obscured such an effect. Possibly in large-animal models this effect can be assessed.

\subsection{TREATMENT MONITORING AND EVALUATION}

Ideally, real-time monitoring of ILC should provide information about the extent of coagulation in relation to the anatomy of the liver during treatment. The intervention may then be prolonged to ensure complete coagulation of the entire tumour, or terminated to prevent damage to vital structures. Interstitially placed thermosensors and light detectors for precise meastrements, are of little use because of the limited number of sample points and the extra trauma of placement. It is likely that real time monitoring by a non-invasive imaging technique is the key to control ILC. Several techniques have been investigated. Especially with magnetic resonance, control is not restricted to morphological 
imaging solely, but physiological parameters can be measured as well.

\section{Ultrasound}

Using ultrasound (US) the tumour can be localised and measured. US enables accurate guided placement of catheters into the appropriate part of the tumour. Real-time imaging of the treated area has been investigated as well but the echogenic area that can be observed during coagulation does not correlate with the coagulative damage (Steger et al, 1992b; van Hillegersberg et al, 1993a; Moller et al, 1997a). The distorted image is caused by formation of bubbles form evaporated tissue water. US is therefore not helpful in evaluating lesions post treatment as the echogenic area becomes heterogeneous within minutes (Amin et al, 1993c; van Hillegersberg et al, 1993a). Follow-up US shows brightly echogenic ellipsoid zones with acoustic shadowing in the treatment area. This gives the treatment area a heterogeneous appearance and thus residual tumour cannot be clearly distinguished (van Hillegersberg et al, 1993a).

\section{Computed tomography}

As for the work-up of surgical candidates for liver resection, triphasic spiral computed tomography (CT) scans are indicated to define the number, location and sizes of metastases prior to laser treatment. CT guided fibre placement is feasible and is preferred to MRI guided insertion because of the good patient access with CT. Contrast-enhanced CT shows the result of laser treatment very clearly as a welldefined, non enhanced area, easily distinguishable from the untreated, thus vascularized, enhancing fumour areas. Contrast enhanced triphasic CT after laser treatment should be considered as the gold standard in treatment evaluation. The optimal time to perform post-ILC CT is between 1 and 4 days, which corresponds with the time at which experimentally induced coagulated lesions have their maximum diameter. Unfortunately at longer intervals post-treatment, CT can not differentiate between, inflammatory change, regenerating liver or recurrent tumour. Due to the X-ray load and insensitivity to soft tissue changes there is no role for real time CT scamming during ILC treatment. 


\section{Magnetic resonance imaging}

Early enthusiasm for CT and US in imaging ILC faded with the advent of powerful and rapid MRI techniques. Initial experience in monitoring laser treatment in patients with hepatic metastases consisted of conventional T1-weighted imaging (Roberts et al, 1994; Vogl et al, 1995; Vogl et al, 1997). Tl-weighted sequences are available on all clinical systems and do not require advanced computer-assisted image-interpretation. The temperature dependence of T1 has been demonstrated in vitro for several tissues (Lewa and Majeska, 1980). However, the relationship between T1 and temperature is not a simple one due to the multifactorial nature of TI (Bottomley et al, 1984; Young et al, 1994a); the temperature dependence of $\mathrm{Tl}$ also varies strongly for different types of tissue (Bottomley et al, 1984). Furthermore, precise measurements of the absolute value of T1 are difficult (Dickinson et al, 1986). Gewiese et al. (1994) visualised a laser heated region, in ex vivo tissue, as a bright area surrounded by a dark border which was found to correspond with an isotherm of $45^{\circ} \mathrm{C}$ $\left(20^{\circ} \mathrm{C}\right.$ increase from starting temperature). More subtle differences, however, were undetectable. Therefore current status of T1 weighted monitoring of heat deposition in tissue does not represent adequate themometry. As an alternative, determination of the molecular diffusion with MRI has been proposed for temperature measurements (Le Bihan et al, 1989; Samulski et al, 1992). With this technique, several parameters quantifying the Brownian motion of free water, are measured. A number of studies in ex vivo tissue reported a very high precision of the temperature measurements. In living tissue, however, the irreversible changes in membrane structures, as the result of tissue coagulation, restrict the water diffusion. These effects may have caused the less precise temperature measurements in in vivo experiments (Cheng and Hemandez, 1992; Samulski et al, 1992; Saint-Jalmes, 1995).

The change of the proton-resonance-frequency (PRF) represents an altemative temperature dependent MR-parameter. This method provides a direct estimate of the temperature changes (De Poorter et al, 1994). In 1995 this concept was elaborated upon, showing the feasibility and accuracy of this subtraction technique (De Poorter et al, 1994). In ex vivo and in vitro models accuracy of phase-shift mapping was within $4^{\circ} \mathrm{C}$ (Kuroda et al, 1997; Sinha et al, 1997). If the accuracy is within the same range in (pre)clinical studies this technique constitutes a good compromise between a simple application, a precise technique and short scanning times. The combination of relatively long acquisition times with the need for each subsequent scan to be made at the position of the reference scan may result in a diminished accuracy. This is especially relevant for application in an organ prone to respiration motion as the liver (Gierada et al, 1995). 


\subsection{CLINICAL EXPERIENCE}

\section{Outcome}

In Table 1.2 the cumulative clinical experience, as published in the literature, is reviewed. The first clinical report was by Hashimoto et al. in ' 85 who treated patients with liver metastases and hepatocellular carcinoma (HCC) at laparotomy. Nd:YAG laser at a low power output of $5 \mathrm{~W}$ was used with relatively long exposure time. A bare-tip laser fibre was used. From this study it was clear that the technique could be performed without major complications. A drop in tumour markers (carcinoembryonic antigen and $\alpha$-foetoprotein respectively) was found. The benefit for the patients remained unclear. In succeeding studies the technique was improved using various combinations of power output and exposure times, and adaptation of the fibre tip to avoid carbonisation. Hahl $e t$ al. (1990) and Huang et al. (1991) actually used hyperthermia, instead of direct coagulation, which means that the temperature was held between $41^{\circ} \mathrm{C}-45^{\circ} \mathrm{C}$ during $10-30$ minutes. A fatal complication was caused by air emboli as a result of interstitial air introduced by cooling of the fibre tip. This method of cooling has been abandoned ever since.

The percutaneous procedure was introduced by Amin et al. (1993c) and adapted later by Nolsoe et al. (1993) and Schroder et al. (1994). Total irradication of small tumours was found. Failures were noted mostly in tumours larger than $2.3 \mathrm{~cm}$; this threshold for complete response was also found in a larger series by Vogl et al. (1995) using single fibre application with a diffusing tip. Amin et al. (1993c) used simultaneous bare-tip-fibre application, necessitating reinsertion and repulling of the fibres to completely cover the tumour. In an updated series, Vogl et al. evaluated the effect on survival after percutaneous ILC in 99 patients with colorectal metastases of the liver (1997). A three-year survival of $42 \%$ with a median survival of 36 months was reported. This was at the expense of an average of 2.9 repeated treatments per tumour (range not stated), both for recurrence in treated tumours as for newly diagnosed tumours (not distinguished). As each study defines its own criteria of selection and evaluation, the findings on tumour response and survival are difficult to compare (Table 1.2). Still, the overall conclusion is allowed that $\Pi \mathrm{C}$ is 1) safe, 2) percutaneously feasible and tolerable and 3 ) effective in destroying small tumours $(<2.5 \mathrm{~cm})$. Larger tumours are variably and incompletely destroyed and recurrence occurred in these lesions. 
Table 1.2 Overview of clinical ILC for hepatic tumours

\begin{tabular}{|c|c|c|c|c|}
\hline Reference & Year & Group characteristics & Technique & Approach \\
\hline Huang et al. & 1991 & $7 \mathrm{HCC} ; 2-4 \mathrm{~cm}$ & Diffusing tip; $2-3 \mathrm{~W}, 20-30 \mathrm{~min}$ & Laparotomy (thermocouple) \\
\hline Nolsoe et al. & 1993 & $16 \mathrm{CRCs} ; 1-4 \mathrm{~cm}$ & Diffusing tip; $4-8 \mathrm{~W}, 5-45 \mathrm{~min}$ & $\begin{array}{l}2 \text { at laparotomy (thermocouple), } 9 \\
\text { percutaneously (US) }\end{array}$ \\
\hline Amin et al. & 1993 & $\begin{array}{l}34 \mathrm{CRCs}, 21 \text { secondaries of mixed origin; } \\
1-15 \mathrm{~cm}\end{array}$ & $4 \mathrm{x}$ bare tips simultaneously; $2 \mathrm{~W}, 7 \mathrm{~min}$ & Percutaneously (US) \\
\hline Schroder et al. & 1994 & $\begin{array}{l}2 \mathrm{HCC} ; 3-7 \mathrm{~cm}, 2 \text { breast, } 6 \mathrm{CRCs} ; 0.5-7 \\
\mathrm{~cm}\end{array}$ & Bare tip; $2-6 \mathrm{~W}, 8-15 \mathrm{~min}$ & Laparotomy (US) \\
\hline Dowlatshahi et al. & 1995 & $\begin{array}{l}45 \text { in } 20 \text { patients; HCC and CRCs; } \\
\text { diameter NS }\end{array}$ & Bare tip; 5-10W, $7 \mathrm{~min}$ & Laparotomy (US) \\
\hline Tranberg et al. & 1996 & $\begin{array}{l}3 \mathrm{HCC}, 18 \mathrm{CRCs}, 1 \text { pancreatic secondary; } \\
1-10 \mathrm{~cm}\end{array}$ & $\begin{array}{l}\text { Frosted sapphire tip; } 6 \text {-10W, } 5 \mathrm{~min} \text { with complete } \\
\text { hepatic inflow occlusion }\end{array}$ & Laparotomy (US) \\
\hline Gillams et al. & 1997 & 148 secondaries in 55 patients; $1-6 \mathrm{~cm}$ & $2 \times 4$ bare tips simultaneously; $2 \mathrm{~W}$ per fibre, $7 \mathrm{~min}$ & Percutaneously (US and MRI) \\
\hline Vogl et al. & 1997 & $282 \mathrm{CRCs}$ in 99 patients: $<5 \mathrm{~cm}$ & Diffusing tip; $10 \mathrm{~W}, 10-20 \mathrm{~min}$ & Percutaneously (MRI) \\
\hline
\end{tabular}

Legend to Table 1.2: $\mathrm{CR}$, complete response; $\mathrm{CRCs}$, colorectal cancer secondaries; $\mathrm{CT}$, computed tomography; HCC, hepatocellular carcinoma; MRI, magnetic resonance imaging; NS, not stated; PD, progressive disease; PR, partial response; SD, stable disease; US, ultrasound; W, watts; min, minutes. 
Table 1.2 (continued)

\begin{tabular}{|c|c|c|c|c|c|}
\hline Reference & Year & Group characteristics & Tumour response at 6 months & Mean (range) of no of treatments & Complications \\
\hline Huang et al. & 1991 & $7 \mathrm{HCC} ; 2-4 \mathrm{~cm}$ & CT: $40 \% \mathrm{CR}, 40 \% \mathrm{PD}$ & Yes: $1.1(1-2)$ & Mild pain \\
\hline Nolsoe et al. & 1993 & 16 CRCs; $1-4 \mathrm{~cm}$ & $\begin{array}{l}\text { US: } 75 \% \mathrm{CR}(\text { mean } 2.4 \mathrm{~cm}) 25 \% \mathrm{SD} \\
\text { (mean } 3.4 \mathrm{~cm})\end{array}$ & No & $\begin{array}{l}\text { Mild pain, temperature } \uparrow \text {, } \\
\text { pleural effusion }\end{array}$ \\
\hline Amin et al. & 1993 & $\begin{array}{l}34 \mathrm{CRCs}, 21 \text { secondaries of mixed } \\
\text { origin; } 1-15 \mathrm{~cm}\end{array}$ & $\begin{array}{l}\mathrm{CT}: 38 \% \mathrm{CR}, 44 \% \mathrm{SD} \text {, failures at }>4 \\
\mathrm{~cm} \text { in diameter }\end{array}$ & Yes; $3.2(1-8)$ & $\begin{array}{l}\text { Severe pain, subcapsular } \\
\text { haematoma, pleural effusion, } \\
\text { temperature } \uparrow\end{array}$ \\
\hline $\begin{array}{l}\text { Schroder et } \\
\text { al. }\end{array}$ & 1994 & $\begin{array}{l}2 \mathrm{HCC} ; 3-7 \mathrm{~cm}, 2 \text { breast, } 6 \mathrm{CRCs} ; \\
0.5-7 \mathrm{~cm}\end{array}$ & $\mathrm{CT}: 8 \% \mathrm{CR}, 58 \% \mathrm{SD}, 33 \% \mathrm{PD}$ & Yes; $1.8(1-4)$ & $\begin{array}{l}\text { Biloma, vasovagal reaction, } \\
\text { shoulder pain }\end{array}$ \\
\hline $\begin{array}{l}\text { Dowlatshahi } \\
\text { et al. }\end{array}$ & 1995 & $\begin{array}{l}45 \text { in } 20 \text { patients; } \mathrm{HCC} \text { and } \mathrm{CRCs} \\
\text { diameter NS }\end{array}$ & $\begin{array}{l}\mathrm{CT}:<2.5 \mathrm{~cm} 68 \% \mathrm{CR},>2.5 \mathrm{~cm} 0 \% \\
\mathrm{CR}\end{array}$ & No & NS \\
\hline $\begin{array}{l}\text { Tranberg et } \\
\text { al. }\end{array}$ & 1996 & $\begin{array}{l}3 \text { HCC, } 18 \text { CRCs, } 1 \text { pancreatic } \\
\text { secondary; } 1-10 \mathrm{~cm}\end{array}$ & $\mathrm{CT}: \mathrm{CR} 16 \%, 38 \% \mathrm{PR}, 46 \% \mathrm{SD} / \mathrm{PD}$ & No & 1 fatal multi organ failure \\
\hline Gillams et al. & 1997 & $\begin{array}{l}148 \text { secondaries in } 55 \text { patients; } 1-6 \\
\mathrm{~cm}\end{array}$ & $\mathrm{CT}: 15 \% \mathrm{CR}, 38 \% \mathrm{PD}$ & Yes; $2.2(1-12)$ & NS \\
\hline Vogl et al. & 1997 & 282 CRCs in 99 patients; $<5 \mathrm{~cm}$ & $\begin{array}{l}\text { MRI: } 42 \% \text { cumulative } 3 \text { year survival } \\
\text { rate. Tumour response NS }\end{array}$ & Yes; $8(1-36)$ & $\begin{array}{l}\text { Nausea, right subphrenic } \\
\text { haemorrhage }\end{array}$ \\
\hline
\end{tabular}

Legend to Table 1.2: CR, complete response; CRCs, colorectal cancer secondaries; CT, computed tomography; HCC, hepatocellular carcinoma; MRI, magnetic resonance imaging; NS, not stated; PD, progressive disease; PR, partial response; SD, stable disease; US, ultrasound; W, watts; min, minutes. 


\section{Complications}

Serious complications in these clinical case-series studying $\mathrm{LC}$ (without flow occlusion) are seldom seen (Table 1.2). Post treatment pain at the insertion site, pleural effusion or pain at the shoulder as a result of diaphragmatic inritation do occur. Bilomas or haemorrhages requiring external drainage have not yet been encountered. One case of the development of a fatal BuddChiari syndrome the day after laser treatment has been reported by Bown (personal communication, 1996), emphasising accurate monitoring of laser-induced effects near vital intrahepatic structures such as the hepatic veins. Tranberg et al. (1996) reported a fatal case of multi organ failure after laser treatment of a patient with a tumour, $8 \mathrm{~cm}$ in diameter, due to massive tumour necrosis. In their series complete occlusion of hepatic flow was applied with the aim of increasing the volume of coagulation. This may be compared to the "cryoshock phenomenon": multi organ failure after treating large tumours with the use of cryosurgery, without flow occlusion (Lee et al, 1997). It remains to be investigated whether the volume of tissue destruction or the application of flow occlusion contributes to the development of the multi organ failure.

\subsection{CONCLUDING REMARKS}

Surgical resection is the only curative treatment currently available for patients with hepatic metastases of colorectal carcinoma and hepatocellular carcinoma. However only $10-20 \%$ of patients are amenable for surgery and the operative morbidity and mortality rates are relatively high owing to associated normal tissue damage. ILC is one the various local treatment modalities, investigated as an alternative to surgical resection, as techniques resulting in local tissue destruction. Since its introduction in '82, $\Pi \mathrm{LC}$ has evolved into an innovative, minimally invasive technique. Animal studies and preliminary clinical studies have shown the safety and feasibility of the technique. As for the other local modalities, the current status of ILC does not yet ensure complete tumour irradication in all cases. It is concluded that improvement should focus on two aspects: increase in the volume of coagulation in a single session and the realisation of real-time monitoring of the laser-tissue interaction. In the next chapter the strategies, hereto investigated in this thesis, are presented. 


\section{Scope, aims and outline of the thesis}

\subsection{SCOPE OF THE THESIS}

Interstitial laser coagulation (ILC) is a technique, which leads to local tissue destruction as a result of thermal effects. Patients with hepatic tumours are one group of patients for which this technique may be a treatment option. The majority of patients with hepatic metastases from colorectal cancer or hepatocellular carcinoma without extrahepatic spread are ineligible for surgery because of anatomical limitations or medical contra-indications for major abdominal surgery. In the previous chapter the possibilities and current limitations of ILC were illustrated. With regard to tumour response the promising results, obtained in animal experiments, could not be confirmed in clinical studies. In tumours larger than approximately $2.5 \mathrm{~cm}$ in diameter the result is variable and tumours are often incompletely destroyed, necessitating repeated treatment. In summary two factors contribute: a) The small volume of tissue that can be coagulated in a single treatment session and b) The lack of reliable monitoring of the laser-induced effects during $\Pi L C$. Thus whilst it is sure that a tumour $>2.5 \mathrm{~cm}$ in diameter is incompletely coagulated the exact boundary of coagulation and vital tumour cannot be depicted. The first problem needs to be solved before the second topic is relevant. But in the end the success of ILC will be determined by the accuracy with which the completeness of the treatment can be assessed. Various strategies have been strutted out to improve these two inseparable topics and several are investigated in this thesis.

\subsection{OBJECTIVES}

The objectives of the studies described this thesis are

1. To increase the volume of coagulation that can be induced in a single session by:

a. Improving the light delivery equipment (fibre tip and multiple fibre application).

b. Eliminating the cooling effect of the organ perfision. 
2. To improve monitoring of the laser-induced effects, by investigating:

a. The critical isotherm for coagulation.

b. The correspondence between this isotherm and non-invasive thermometry.

\subsection{OUTLINE}

In part II the first objective is addressed. Chapter 3 compares a newly developed fibre tip with the conventional bare tip fibre. Four of these new fibres are used simultaneously in chapter 4 to test mathematical simulations, which predict a synergistic effect in the centre of 4 fibres. As both studies are performed in ex vivo porcine liver, the objective in chapter 5 is to evaluate in pigs these adaptations to the light delivery equipment. Three conditions of liver blood flow are compared with respect to the magnitude of coagulation.

In part III the interaction between the thermal laser effect and the tissue is addressed. By observation at several time points after ILC, the course of the lesions is studied in chapter 6 . In chapter 7 the very same laser parameters are used in ILC of human hepatic tumours. With new histo-enzymatical methods the cellular vitality directly following ILC is determined. Chapter 8 describes studies to the isotherm at which tissue coagulation occurs. In chapter 9 this line of investigations is continued: The same isotherm is determined with magnetic resonance and compared with the macroscopical area of coagulation in order to develop a non-invasive monitoring device. Additionally the influence of motion artefacts is studied. In the final pat IV the respective findings are discussed and integrated. Several aspects for clinical application are considered. In chapter 11 the joint conclusions of this thesis are summarised. 



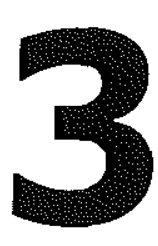

\section{Heat resistant cylindrical diffuser for Interstitial laser coagulation: Comparison with a bare-tip fibre in ex vivo porcine liver}

J Heisterkamp, R van Hillegersberg, EL Sinofsky \& JNM IJzermans. Lasers in Surgery and Medicine 1997, 20; 304-309.

\subsection{INTRODUCTION}

Interstitial laser coagulation (ILC) is a new method of producing localised tissue destruction that may be used to eliminate solid tumours such as hepatic maligiancies (Masters and Bown, 1992). The laser light is delivered to the tumour by one or more optical fibres that are inserted directly into the tissue. The type of fibre tip creating the largest lesion size is still a matter of discussion (Harries et al, 1994; van Hillegersberg et al, 1995). Traditionally, a so called bare-tip fibre is used (Figure 3.1). With this fibre, the emission of light and hence the generated heat is concentrated around the tip (point light source), causing high temperatures that result in carbonisation. The blackish material surrounding the tip absorbs the laser light strongly and suppresses its transmission into the tissue. Thus, a charred fibre acts as a point heat source, and the size of the induced coagulation depends entirely on heat diffusion from the tip toward the tissue. In contrast, a cylindrical diffusing fibre-tip produces a homogeneous light emission over its light diffusing length (distributed light source). By that, the emitted laser light is equally distributed into the tissue, and the induced coagulation depends on light transmission followed by heat production upon absorption in the tissue.

Previous experimental comparisons between both fibre tips showed that at a lower laser output and time, the point light source produced a larger lesion (with charring) than the distributed light source (without charring) (Wyman et al, 1992; Amin et al, 1993b; Wyman et al, 1994). However, the temperature gradient from fibre tip into the tissue is much less steep for a distributed light source than for a point light source (Roggan and Müller, 1994). Furthermore, it requires a higher laser output power for the distributed light source than for the point light source to reach the coagulation temperature (Roggan and Müller, 1994).

We hypothesise that a distributed light source can potentially heat a larger area from the fibre tip 
and accordingly reach a larger coagulation volume than a bare-tip fibre. Monte-Carlo simulations, using the optical and thermal properties of native and coagulated tissue, support this hypothesis (Roggan et al, 1994; Roggan and Müller, 1994). However, there are no supporting experimental data, as the fibre tips have not been tested at their individual optimal laser settings. Furthermore, until recently, the available cylindrical diffusing fibre tips were too large in diameter and temperature unstable. We developed a diffusing tip with the same diameter as the standard $600 \mu \mathrm{m}$ optical fibre that withstands temperatures up to $300^{\circ} \mathrm{C}$. For a standardised comparison of both fibre tips, we designed an experiment using porcine livers in which the bare-tip fibre and cylindrical diffuser were studied at various combinations of laser output and exposure time. In this way the biological effects of both fibres could be assessed at the optimal individual laser settings.

\subsection{MATERIALS AND METHODS}

\section{Optical fibres and construction of cylindrical diffuser}

Laserlight was transmitted through glass fibres (core diameter $600 \mu \mathrm{m}$, outer diameter $1.65 \mathrm{~mm}$ ) with either a diffusing end of $21 \mathrm{~mm}$ or a bare-tip fibre with a freshly cleaved end and the most distal coating stripped for $15 \mathrm{~mm}$ (Figure 3.1). The cylindrical diffuser used for these experiments [Lightstic ${ }^{\text {TM }}$, Cardiofocus, West Yarmouth, MA USA] was designed to withstand temperatures of $300^{\circ} \mathrm{C}$. The diffusion region of the fibre-optic catheter consisted of epoxy containing scatterers, encased in fluoropolymer sheath. An end mirror was sharpened to ease insertion and was coated to reflect all of the $1064 \mathrm{~nm}$ light, enhancing uniformity and preventing light from propagating out of the end of the tip. Transmission was always above $85 \%$, with uniformity of typically $\pm 7 \%$ along the diffusion region. Hot spots which could overexpose areas of tissue, were never found. The tips were tested at $60 \mathrm{~W} \mathrm{CW}$ in water for 60 seconds before use in the experiments.

\section{Laser application}

A Nd:YAG laser [4060 N, MBB-Dornier, Munchen, Germany] with a wavelength of $1064 \mathrm{~nm}$ was used. Illumination was performed with a laser output ranging from 3-9 W and exposure times of 618 minutes. As in pilot experiments the cylindrical diffusing-tip carbonised at $6 \mathrm{~W}$ and $12 \mathrm{~min}$ $(4320 \mathrm{~J})$, this combination of settings was only tested for the bare-tip fibre. 


\section{Liver tissue}

Porcine livers were preserved at $-20^{\circ} \mathrm{C}$ and gradually defrosted before each experiment. The different lobes were cleaved into two slabs of $2.5 \mathrm{~cm}$ thick (Wyman, 1993; Roberts et al, 1994). Prior to illumination the liver slabs were gradually warmed between two warm water mattresses until a core temperature of $37^{\circ} \mathrm{C}$ was reached. Liver core temperature was recorded with a $\mathrm{Ni}(\mathrm{Cr})$ NiK thermocouple [Thermodig N800, AIS, France].

\section{Procedures}

A fibre was placed between two liver slabs at a distance of at least $3 \mathrm{~cm}$ from a previously produced lesion. To keep the core temperature of the liver at $37^{\circ} \mathrm{C}$ during laser application, the top slab was covered with a warm water mattress. To ensure good contact between the two slabs a weight of $1 \mathrm{~kg}$ was placed on top. After illumination the top slab was removed and length (parallel to the fibre) and diameter (perpendicular to the fibre) of the coagulated lesion were measured with callipers. The extent of the lesion was estimated as the macroscopically detectable (whitish) colour change compared to normal coloured liver parenchyma. Each lesion was checked for carbonisation.

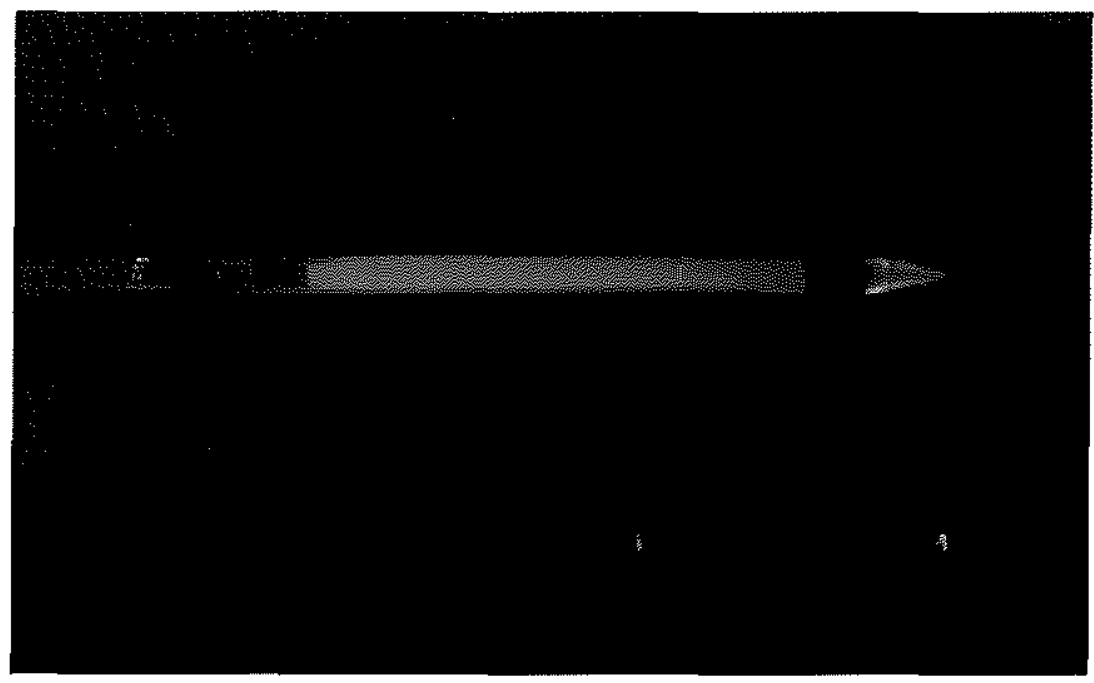

Figure 3.1 Two laser fibres for interstitial laser coagulation emitting pilot laser light from a cylindrical diffusing-tip fibre (above) and a bare-tip fibre. 


\section{Tissue Temperature}

Temperature distribution during illumination was measured at the combination of $6 \mathrm{~W}$ for 6 minutes (2160 Joules). Fibre-optic thermosensors with a diameter of $0.5 \mathrm{~mm}$ were coupled to a Luxtron thermometry unit [Luxtron Corp., Santa Clara, CA, USA] and inserted parallel to the laser fibre into the tissue at axial distances of 3,8 and $13 \mathrm{~mm}$ from the fibre tip. Temperature was sampled every second and stored using a computer. Self adsorption was tested in air and determined as an immediate temperature rise of $3-4^{\circ} \mathrm{C}$ at $1 \mathrm{~cm}$ distance. Influence on the temperature measurements because of selfadsorption was not taken into consideration in the porcine liver experiments, as this immediate temperature rise could not be demonstrated.

\section{Statistics}

The values are expressed as mean \pm standard error of the mean (SEM). For each fibre type, a multiple linear regression model for length and diameter was fitted including power and energy as explanatory variables. Possible deviations from linearity for the explanatory variables were tested, which resulted in adding a quadratic term for power in the regression equation for diameter for the diffuser-tip fibre, but not for the bare-tip fibre. Deviation from parallelism of the regression lines for length of each fibre type was tested using Student's $t$-test on the regression coefficient. To test for variability in lesion size of the repeated measurements between the two fibres a F-test i.e. variance ratio test, was performed with the residual mean of squares. Values were considered statistically significant at $P$ values $<0.05$.

\subsection{RESULTS}

\section{Macroscopy}

Typical bare-tip fibre lesions were irregular in shape with central charring and sometimes charring parallel along the fibre (Figure 3.2). Polishing of the freshly cleaved bare-tip fibres did not enhance reproducibility of lesion size and shape. The cylindrical diffuser-tip lesions were characteristically ellipse in shape (Figure 3.2). With this tip slight charring was found when $9 \mathrm{~W}$ for $6 \mathrm{~min}$ was applied $(3240 \mathrm{~J})$, however no destruction of the fibre tip occurred. Damage because of massive charring occurred when $5 \mathrm{~W}$ was applied for $18 \mathrm{~min}(5400 \mathrm{~J})$. 


\section{Lesion size}

The relationship between length and diameter of the lesion for energy applied is given in Figure 3.3 (bare-tip fibre) and Figure 3.4 (diffusing-tip fibre). The lines of various power output have a similar slope for increasing energy applied. For both types of fibres, length and diameter also enlarged with increasing power. This incline in lesion size was significantly larger for the cylindrical diffuser, as computed with the multiple linear regression model (length $P=0.048$, diameter $P=0.042$ ).

\section{Tissue temperature}

Table 3.1 shows the temperature distribution measured at a distance of 3,8 and $13 \mathrm{~mm}$ from the fibre tip. Close to the fibre, tissue temperature was markedly higher for the bare-tip fibre, $260^{\circ} \mathrm{C}$ (17) versus $123^{\circ} \mathrm{C}(5)$ for the diffusing-tip. At $8 \mathrm{~mm}$, the tissue temperature was comparable for both fibres. At the distance of $13 \mathrm{~mm}$ the mean temperature for the diffusing tip fibre was close to the coagulation temperature $58^{\circ} \mathrm{C}(2)$, whereas for the bare-tip fibre values were more in the range of the starting core temperature $39^{\circ} \mathrm{C}$ (1). The standard errors of the mean (SEM) of the mean temperature values were significantly higher for the bare-tip fibre $(P=0.015)$.

Table 3.1 Mean tissue temperature $\pm \operatorname{SEM}\left({ }^{\circ} \mathrm{C}\right)$ at various exposure times at 3,8 and $13 \mathrm{~mm}$ from the fibre-tip. Tissue was illuminated with $6 \mathrm{~W}$ for 6 minutes $(2160 \mathrm{~J}), n=6$ for each measurement

\begin{tabular}{ccccccc}
\hline & \multicolumn{6}{c}{ Exposure time } \\
\cline { 2 - 5 } distance (mm) & 1 min & 2 min & 3 min & 4 min & 5 min & 6 min \\
\hline bare-tip fibre & & & & & & \\
3 & $95(9)$ & $131(11)$ & $220(20)$ & $249(19)$ & $260(20)$ & $260(17)$ \\
8 & $62(5)$ & $71(4)$ & $84(4)$ & $100(7)$ & $110(4)$ & $115(8)$ \\
13 & $36(1)$ & $38(1)$ & $38(1)$ & $39(1)$ & $39(1)$ & $39(1)$ \\
diffusing-tip fibre & & & & & & \\
3 & $66(3)$ & $91(4)$ & $111(4)$ & $123(5)$ & $124(4)$ & $123(6)$ \\
8 & $46(2)$ & $50(2)$ & $63(2)$ & $74(3)$ & $85(3)$ & $101(6)$ \\
13 & $37(1)$ & $39(1)$ & $43(2)$ & $48(2)$ & $52(3)$ & $58(2)$ \\
\hline
\end{tabular}



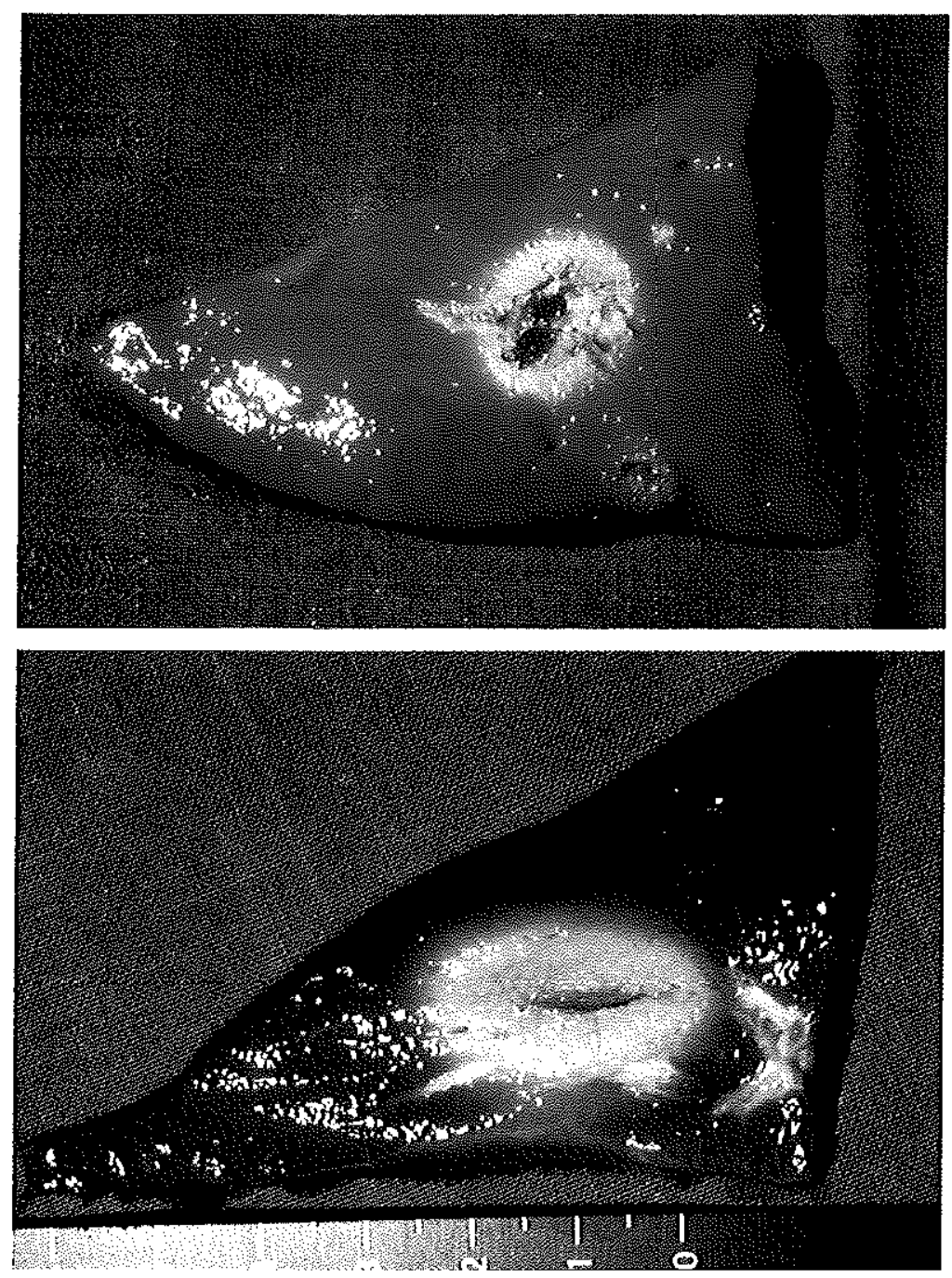

Figure 3.2 Macroscopic view of a typical lesion ( $6 \mathrm{~W}$ for $6 \mathrm{~min}, 2160 \mathrm{~J}$ ) created with the bare-tip fibre (above, length $14 \mathrm{~mm}$; diameter $18 \mathrm{~mm}$ ) and the cylindrical diffusing-tip fibre (under, $34 \times 24 \mathrm{~mm}$ ). 


\section{Factors influencing lesion size}

The lesion size was more reproducible for the diffusing-tip fibre compared to the barc-tip fibre. The variance ratio test was significantly different from $\mathrm{F}=1$, both for length $(\mathrm{F}=2.01, P=0.032)$ and diameter $(\mathrm{F}=2.48, P=0.039)$. For the diffusing-tip, the laser output was a more important factor determining lesion size than exposure time, comparing regression coefficients computed with the multiple linear regression model $\left(b_{\text {power }}=2.078 ; b_{\text {time }}=0.0032\right)$.
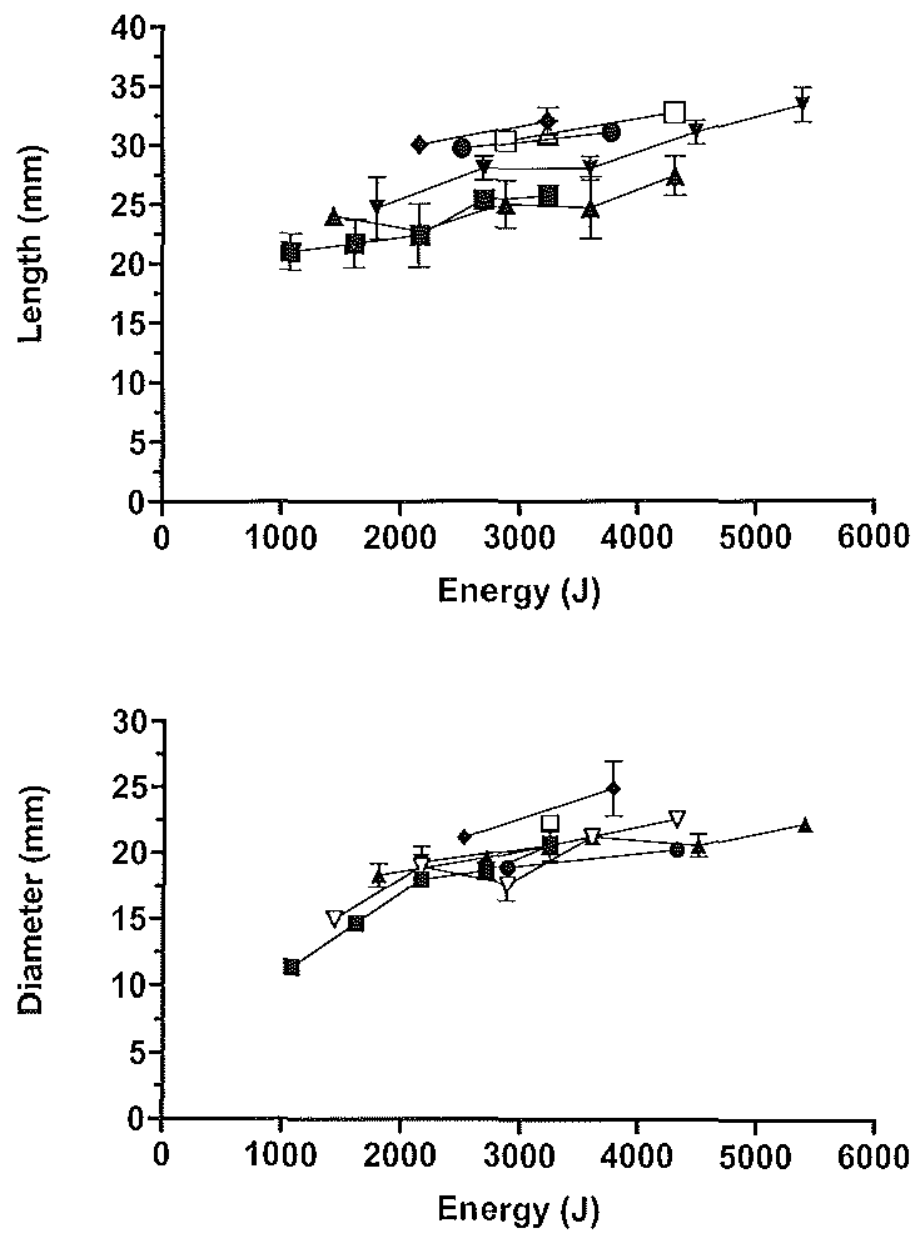

Figure 3.3 Length and diameter of coagulated lesions versus energy applicd for the bare-tip fibre. Each line

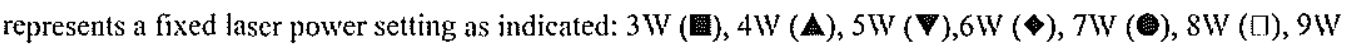
$(\nabla)$. Each point represents the mean \pm SEM of three experimental results. 

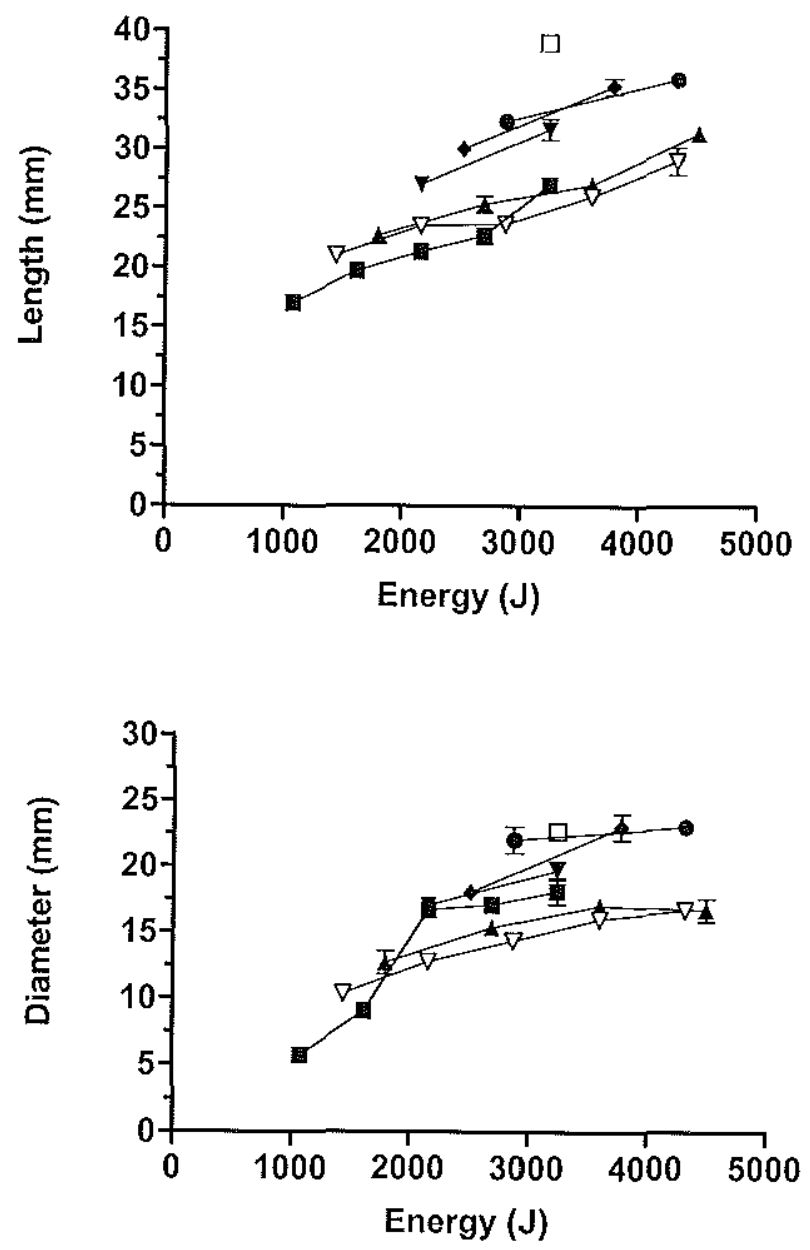

Figure 3.4 Length and diameter of coagulated lesions versus energy applied for the diffusing-tip fibre. Each line represents a fixed laser power setting as indicated: $3 \mathrm{~W}(\mathbf{E}), 4 \mathrm{~W}(\nabla), 5 \mathrm{~W}(\Delta), 6 \mathrm{~W}(\diamond), 7 \mathrm{~W}(\mathbf{V}), \mathrm{WW}$ (๑), 9IV (ப). Each point represents the mean \pm SEM of three experimental results. 


\subsection{DISCUSSION}

Bare-tip fibres, which are easy to make and inexpensive to produce are often used in interstitial laser coagulation. Furthermore has it been suggested that a point light source produces a larger volume of coagulation necrosis than a distributed light source at the same laser output and exposure time (Wyman et al, 1992; Amin et al, 1993b; van Hillegersberg et al, 1995). Our in vitro experiments show that the lesion size at lower laser outputs was larger for the bare-tip fibre than for the cylindrical diffusing-tip fibre. However at higher laser output the size for the diffuser increased, whereas the size of the lesion for the bare-tip fibre seemed to reach a plateau.

Although we used healthy, previously preserved porcine liver, it is most likely that the similar difference between the bare-tip fibre and the diffusing-tip fibre will occur in vivo. However, the thermal and optical properties of tumour tissue may influence the different mechanisms of heating tissue (thermal diffusion versus light transmission and subsequent heat production) in a different way (van Hillegersberg et al, 1993b). It can be hypothesised that the difference in lesion size between the two fibres may be larger in vivo. When using a bare-tip fibre, its mechanism of carbonisation and subsequent thermal diffusion may be more influenced by the cooling produced by blood flow than the heating mechanism of the diffusing-tip (van Hillegersberg et al, 1994).

One of our goals was to determine the maximal lesion size for both fibres at their optimal individual combination of power and exposure duration. From Figure 3.3 it can be noted that the size of the lesion for the bare-tip fibre has reached a plateau at $6 \mathrm{~W}$ for $9 \mathrm{~min}$. Once charring around the baretip fibre has occurred and light penetration into the tissue is limited, further absorption of laser energy is less effective (Matthewson et al, 1987; Thomsen, 1991). Ultimately at higher laser and power settings, lesion size for a cylindrical diffuser also will be limited by the maximal laser light penetration and heat diffusion into the tissue.

The temperature profiles as determined from measurements at three distances from the fibre tip do confirm investigations of Wyman et al. (1993) and calculations of Roggan and Müller (1995). They predicted a higher tissue temperature close to the light fibre for the bare-tip fibre with a steeper temperature gradient towards the tissue periphery compared to the cylindrical diffusing tip. Although the location of the themofibres was critical, we take the view that the 6 repeated measurements give a good indication of the actual temperature profile.

Especially for the cylindrical diffuser, power was a more important factor in determining lesion size than exposure time. Although the amount of encrgy delivered at the combination $5 \mathrm{~W}$ and 15 in $(4500 \mathrm{~J})$ is ligher than for the combination $8 \mathrm{~W}$ and 9 in $(4320 \mathrm{~J})$, the lesion size created by the 
latter was considerably larger. Therefore, in a clinical application, increasing power will be more efficient than prolonging exposure times for destroying larger tumours.

The unpredictability for bare-tip fibre-created lesions could be explained by the variability in the time at which charring occurs which determines the mechanism of heating. This could also account for the larger variation in tissue temperature distribution for the bare-tip fibre measured during illumination. Similar results were reported Harries et al. (1994) in a clinical study on interstitial laser therapy using a bare-tip fibre, as their results were more predictable when using a precharred fibre.

In conclusion, have we found that lesions produced by a cylindrical diffuser were significantly larger and more reproducible than with a bare-tip fibre. These findings imply that ILC should be performed with a cylindrical diffusingntip fibre. Charring should be avoided: 1 to maintain the integrity of the diffusing fibre, 2) to maximise the size of the coagulation necrosis and 3) to ensure reproducible results. An important additional advantage of a cylindrical diffuser is that its length can be adapted to the relevant tumour size. By that tumours of $3-4 \mathrm{~cm}$ can be treated over their entire diameter in one session, whereas the bare fibre needs to be pulled back several times (Amin et al, 1993a). The diffusing tip may therefore also increase the preciseness of tumour destruction. 


\section{Simultaneous multiple fibre application to increase the volume of Interstitial laser coagulation using an optical beamsplitter}

J Heisterkamp, R Hillegersberg, EL Sinofsky \& JNM IJzermans. Lasers in Medical Science, 1999, $14 ; 216-220$.

\subsection{INTRODUCTION}

Ever since the introduction of interstitial laser coagulation (ILC) efforts have been made to increase the volume of coagulation produced by this technique (Castren-Persons et al, 1992; Matsumoto et al, 1992; Wyman et al, 1992; Amin et al, 1993b; Prapavat et al, 1996). Recent clinical series show that small hepatic metastases $(1.6-2.6 \mathrm{~cm}$ ) can be destroyed effectively (Amin et al, 1993c; Vogl et al, 1995; Tranberg et al, 1996). However, tumours larger than approximately $2.5 \mathrm{~cm}$ are variably and incompletely destroyed. In chapter 3 , it was described that by using a single optical fibre with a heat resistant cylindrical diffusing tip, it is possible to produce spherical lesions of $3.5 \mathrm{~cm}$ in diameter. Most hepatic metastases originating from colorectal cancer, however, are larger in diameter at diagnosis (Stangl et al, 1994). Further improvements in the technique are a prerequisite for effective clinical application. Tranberg et al. (1996) and Amin et al. (1993b) described the use of multiple puncturing and redrawing the laser fibre during application in order to increase the volume of coagulation. This has two disadvantages: Firstly destruction is inaccurate and secondly the technique is time-consuming.

Simultaneous multiple fibre application, using 4 fibres introduced in a square fashion into the tissue, has been proposed as an altemative strategy. Synergism between multiple fibres was shown by Davis et al. (1989) in a mathematical model. Both a reduced heat dissipation in the centre of the fibres and an additive effect at the borders of the individual lesions account for this effect (Svaasand et al, 1985). To apply 4 fibres simultaneously, several beamsplitting devices have been developed. Although beamsplitters are no novelty in applied optical physics, their use in ILC has been limited to several prototypes (Steger et al, 1992a; Bosman, 1993). Details regarding design of these splitters are not described in the literature. In essence, construction was based on either 
semitransparent mirrors or by using fused fibres solded on the feeding fibre respectively. Both types, however, could not withstand laser output powers higher than $10 \mathrm{~W}$ due to power loss with subsequent overheating (Bosman, 1993; Bown, 1996). We constructed a 1 × 4 beamsplitter based on optical principles that ensures high transmission efficiency for high power laser application.

When using simultaneous multiple fibre application, the distance at which the fibres are positioned determines whether the synergistic thermal effect between the fibres occurs (Steger $e t a l, 1992 \mathrm{a}$; Wyman, 1993). To determine the optimal combination, the histological effects of laser power and mutual fibre distance were assessed in ex vivo porcine liver. In this chapter, both the construction of- and the experimental results with. the beamsplitter are reported.

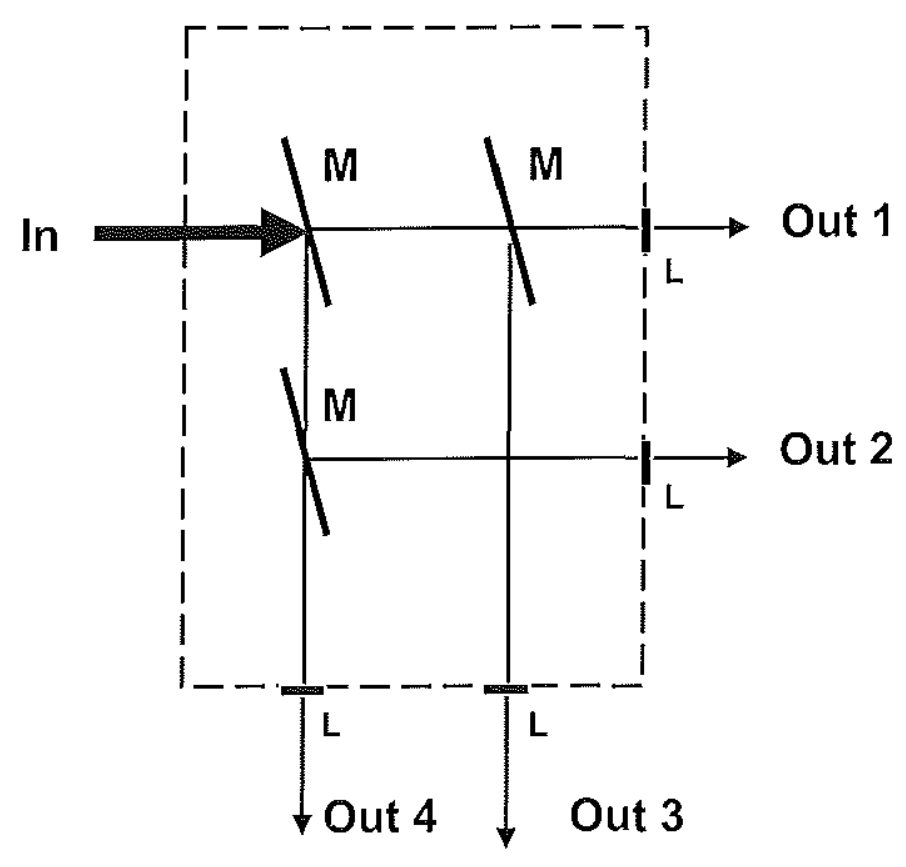

Figtre 4.1 Schematic diagram of the beamsplitting device, in its aluminum encasement of $7.5 \times 6.5$ $\mathrm{cm}$. With $M$ the 3 semi- transparent mirrors (50-50\%) and with $\mathbf{L}$ the collimating and demagnifying lenses (70\%). To the ports the feeding (IN) and fed fibres (OUT 1-4), coupled using standard SMA-905 connections. See Table 4.1 for data of transmission efficiency and output variation. 


\subsection{MATERIALS AND METHODS}

\section{Beamsplitter and laser fibres}

Fibres with a cylindrical diffusing tip of $2 \mathrm{~cm}$ length, outer diameter $1.65 \mathrm{~mm}$ and core diameter $600 \mu \mathrm{m}$ were used [Lightstic ${ }^{\mathrm{TM}}$, Cardiofocus, West Yarmouth, Ma, USA]. A homogeneous light emission over the entire diffusing part is ensured, as described in chapter 3 . The four fibres were fed by a custom built $1 \times 4$ beamsplitter [Lightstic $4 \mathrm{X}^{\mathrm{TM}}$, Cardiofocus], coupled by standard SMA905 connectors. The beamsplitter consisted of three semi-transparent (50/50) dielectric coated mirrors, splitting the laser output into 4 approximately equal beam trains (Figure 4.1). The back surfaces of the custom-built mirrors were antireflection coated $(\mathrm{R}<0.5 \%)$. The output from the feeding fibre was collimated with an antireflective coated lens. This beam was then split into 2 beams by positioning the semi-transparent mirrors at a 45 degrees angle to the incoming beam. These two beams were then split again into two beam trains, each with 2 more 50/50-beamsplitting mirrors. Once the 4 collimated beams were generated, they were individually re-imaged onto each of 4 output ports with a demagnification of $70 \%$, using anti-reflection coated lenses. The beamsplitter and imaging optics were permanently affixed in place, such that no precision positioning was necessary afterwards. The beamsplitting components were encased in an aluminium box measuring $7.5 \times 6.5 \mathrm{~cm}$ (Figure 4.1 ).

Nd:YAG light of $1064 \mathrm{~mm}$ [KTP/YAG 814, Laserscope, San José (CA), USA] was guided to the beamsplitter using a coated $600 \mu \mathrm{m}$ connecting cable. The fibre from the laser had a numerical aperture $(\mathrm{NA}=0.22)$ smaller than the numerical aperture of the devices $(\mathrm{NA}=0.37)$.

\section{Measurement of transmission efficiency and output variation of beamsplitter}

Measurements were made to quantify the net transmission of the unit as a whole and to measure the variation between the 4 channels. Power from the connecting fibre from the laser and output directly from the 4 ports were measured with a calibrated power meter [Labmaster E Thermopile, Coherent, Santa Clara (CA), USA]. Power from the 4 channels was then summed and divided by the original input power to obtain the net transmission percentage. Variation of the power between output channels was calculated relative to the total output power. 
Table 4.1 Power output (in Watts) from the 4 channels of the beamsplitter for several laser input power levels.

\begin{tabular}{ccccccc}
\hline P input & port 1 & port 2 & port 3 & port 4 & variation (\%)广ं & $\begin{array}{c}\text { transmission * } \\
(\%)\end{array}$ \\
\hline 5.3 & 1.2 & 1.2 & 1.1 & 1.1 & 7.0 & 87 \\
10.3 & 2.4 & 2.3 & 2.0 & 2.1 & 7.5 & 85 \\
19.1 & 4.7 & 4.6 & 4.00 & 4.0 & 8.0 & 90 \\
23.6 & 5.8 & 5.5 & 4.8 & 4.9 & 9.5 & 89 \\
27.1 & 6.7 & 6.5 & 5.6 & 5.7 & 8.9 & 90 \\
32.3 & 7.5 & 7.6 & 6.8 & 6.9 & 9.0 & 89 \\
\hline
\end{tabular}

Notes: $\uparrow$ Variation of the power between output channels was calculated relative to the total output power.

* Power from the 4 channels was summed and divided by the original input power to obtain the net transmission percentage.

\section{Laser application and procedures}

Output power was varied from $4-7 \mathrm{~W}(\mathrm{CW})$ per fibre $(2-3.5 \mathrm{~W} / \mathrm{cm}$ diffuser length, $20-35 \mathrm{~W}$ output from the laser). By adjusting exposure time $[258 \mathrm{sec}-450 \mathrm{sec}(4,5,6$ and 7 minutes respectively)], energy per fibre was constant at $1800 \mathrm{~J}$. For each power setting, distances between the 4 fibres, positioned in ex vivo porcine liver, were varied from $1-4 \mathrm{~cm}$. Each combination of power and fibre distance was replicated five fold.

Porcine livers were preserved at $-20^{\circ} \mathrm{C}$ and gradually defrosted overnight. Lobes were then separately warmed, using warm water mattresses, to approximately $28^{\circ} \mathrm{C}$ (varying between $26^{\circ} \mathrm{C}$ and $29^{\circ} \mathrm{C}$ ) and then used for 1 or 2 laser applications. Liver core temperature was recorded with a $\mathrm{Ni}(\mathrm{Cr})$-NiK thermocouple [Thermodig N800, AIS, France]. Small bore cannulas [Adsyte 14G, Becton Dickinson, Spain] were positioned at the corners of a square, the side being the experimental distance, using a custom built template. After inserting the fibres the cannulas were redrawn.

\section{Evaluation}

After laser application, the liver lobe was dissected with a long bladed knife, orthogonal to the insertion of the 4 fibres in slices of $0.5 \mathrm{~cm}$. The coagulated volume was estimated as the 
macroscopically detectable (whitish) colour change, compared to normal coloured liver parenchyma. Height $(h)$ of the lesion and diameter of the largest coagulated plane $(w)$ were measured with callipers. Volume was estimated using the volume of a rotational ellipsoid: $\mathrm{V}=h \mathrm{x}$ $w^{2} \times \pi / 6$ (Carlsson et al, 1983). When no confluence of coagulated zones had occurred, the total coagulated volume was calculated by summing the volumes of the 4 zones. Each lesion was checked for carbonisation.

\section{Statistical evaluation}

Values are expressed as mean \pm standard deviation (SD). Two-way analysis of variance (ANOVA) was used for 2 independent, but crossed, explanatory variables: distance ( 6 levels) and power output (4 levels) on 1 response variable: coagulated volume. Natural log-transformation was used to stabilise the experimental error of the response, assuming that the relative error was constant, rather than the absolute error. The response was regressed onto polynomials for both power and distance (Venables and Ripley, 1994). Values were considered statistically significant at $P$ values $<0.05$.

\subsection{RESULTS}

\section{Beamsplitter}

Transmission efficiency of the beamsplitter was always above $85 \%$ [88.3\% (1.3)]. Variation in output between the 4 output channels was always below $10 \%[8.0 \%(0.51)]$ (Table 4.1 ). Overheating of the beamsplitting device or breakdown of the diffusing fibres never occurred, neither were macroscopic signs of carbonisation detected at the diffusing tips.

\section{Macroscopy and lesion diameter}

Three typical lesions, produced with $7 \mathrm{~W}$ per fibre $(3.5 \mathrm{~W} / \mathrm{cm}$ diffuser length) with the fibres at 3 different mutual fibre distances, are shown in Figure 4.2. With 2.5,3 and $4 \mathrm{~cm}$ distance between the fibres confluence of the 4 zones of coagulation never occurred. Thus comparable volumes of coagulation were found for these three distances at each respective power level. At distances 1, 1.5 and $2 \mathrm{~cm}$ between the fibres, confluence of the 4 zones always occurred. With $1 \mathrm{~cm}$ between the fibres, carbonisation was found in the centre of the square. The largest volume of coagulation, 44.5 $(2.1) \mathrm{cm}^{3}$ (lesion diameter of $5.1(0.4) \mathrm{cm}$ ), was produced with $7 \mathrm{~W}$ and $2 \mathrm{~cm}$ mutual fibre distance. Both distance and power were independent factors for coagulated volume $(P<0.001$ and $P<$ 0.0001 respectively), indicating that the various levels of power and distance resulted in 
significantly different coagulated volumes. Interaction of both factors was found as well $(P<$ 0.001 ), indicating synergism between duration and magnitude of the exposition. Residuals were examined and it was found that the model fitted the data.

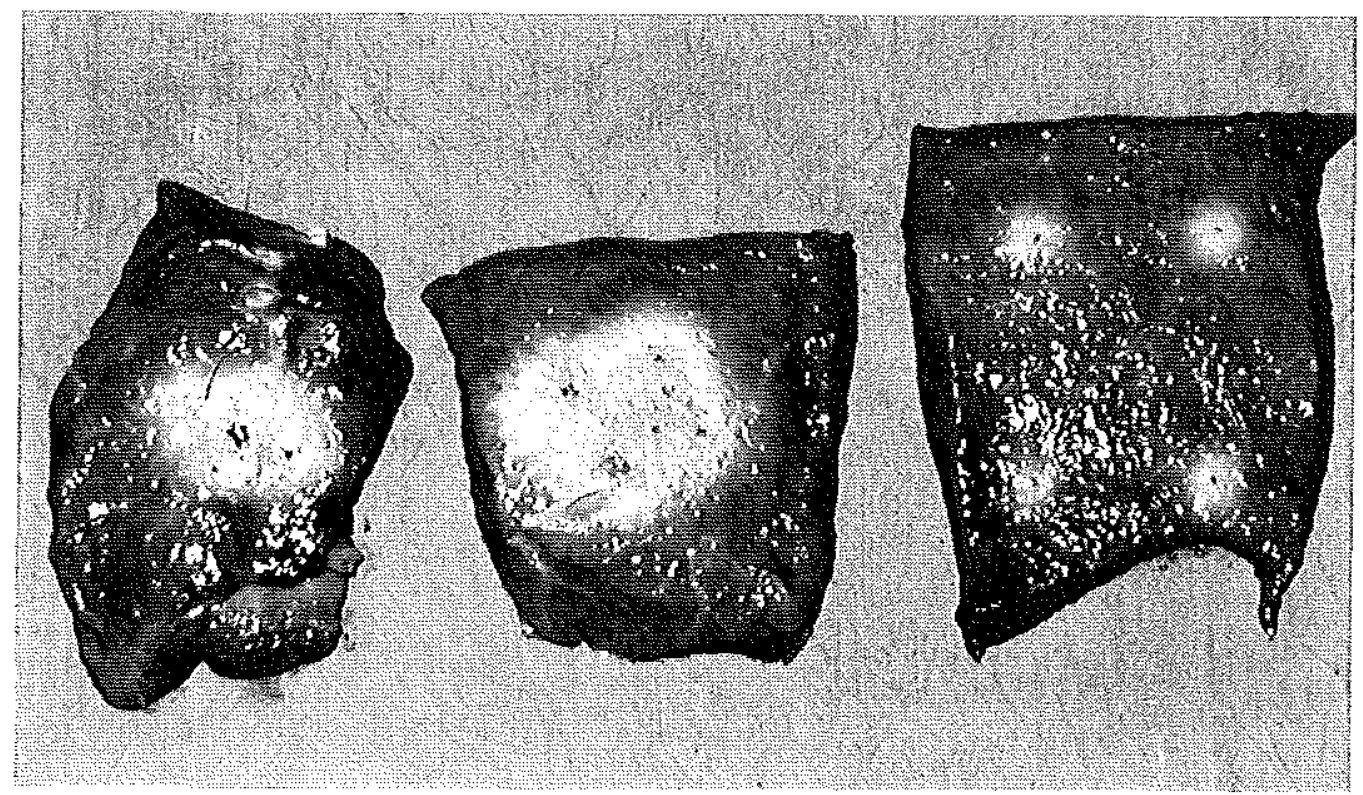

Figure 4.2 Typical lesions produced by simultaneous multiple application of 4 interstitial tibres with $7 \mathrm{~W}$ per fibre $(1800 \mathrm{~J})$ in ex vivo porcine liver. Mutual fibre distance $(d)$ is respectively $1 \mathrm{~cm}$ (left), $2 \mathrm{~cm}$ (middle) and $4 \mathrm{~cm}$ (right). With $d=1 \mathrm{~cm}$, carbonisation in the centre between the 4 fibers was always found. Mean total volumes (SD) are respectively $16(0.6), 44(2.1)$ and $15.4(0.7) \mathrm{cm}^{3},(n=5$ for each combination).

\subsection{DISCUSSION}

In this study, we assessed the optimal combination of laser power and distance for 4 interstitial laser fibres, applied simultancously with a custom built high-power beamsplitter. The use of beamsplitting devices for high power Nd:YAG light has been reported earlier (Frank and Hessel, 1990; Steger et al, 1992a; Bosman, 1993). Until now, however inefficient beamsplitting devices were used with a large output variation between the channels. We focused in this study on improving these two items in a newly developed beamsplitter. Accurate in-line positioning of the 
semi-transparent mirrors was used in combination with true $50 / 50$ splitting mirrors. In addition, the anti-reflection coatings decreased the loss of light at each component. The net transmission percentage above $85 \%$, infers a very high coupling ratio of the re-imaging, as well as very little loss in the beamsplitter mirrors and imaging lenses. The high efficiencies obtained are crucial in the operation of the beamsplitter at high power levels for extended duration, preventing excess heating. No cooling was necessary with this beamsplitter.

High power beamsplitters are a prerequisite when using fibres with a cylindrical diffusing tip to maintain adequate power over the entire diffusing length. The homogeneous emission of the light from the tip over a certain length prevents carbonisation. Larger lesions are produced than with a conventional bare tip fibre, however, at the cost of higher powers (chapter 3 ).

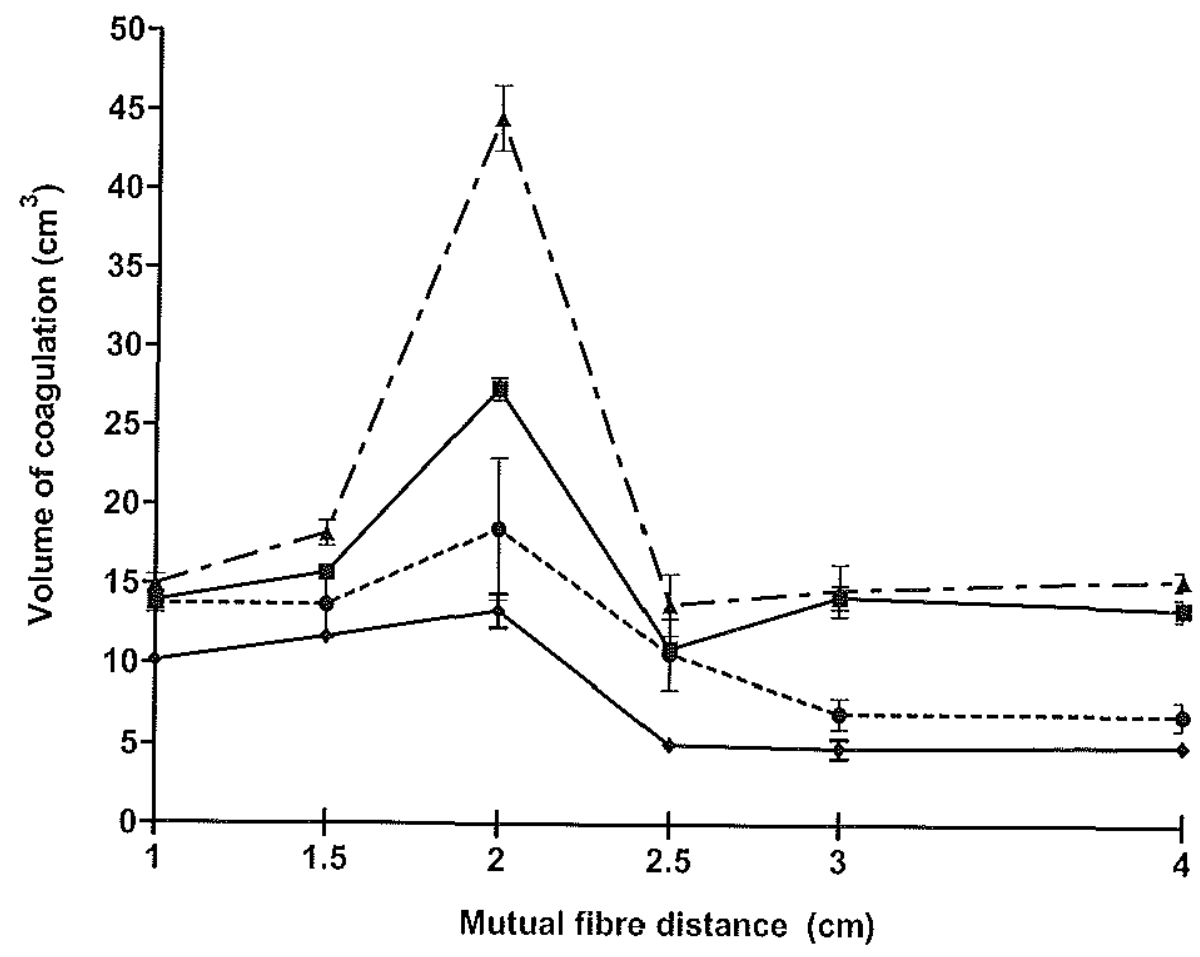

Figure 4.3 Volumes of coagulation produced with simultaneous application of 4 interstitial fibres in ex vivo porcine liver. Laser power was varied between $4 \mathrm{~W}(\$), 5 \mathrm{~W}(\mathbf{0}), 6 \mathrm{~W}$ (圈) and $7 \mathrm{~W}(\mathrm{~A})$. By varying exposure time, energy was constant at $1800 \mathrm{~J}$ per fibre. Points and bars represent the mean and SD of 5 experimental results. 
In this ex vivo porcine liver model, we found an optimal synergistic effect at $7 \mathrm{~W}$ for 258 seconds (1800 J per fibre) with $2 \mathrm{~cm}$ between the fibres (coagulation of $5.1 \mathrm{~cm}$ in diameter). The synergistic effect of simultaneous application results in 3 fold enlargement of the volume of coagulation: volume of coagulation produced with $2 \mathrm{~cm}$ distance compared to the result with the same laser parameters with $4 \mathrm{~cm}$ between the fibres. In the previous chapter with a single fibre in ex vivo porcine liver, the largest lesions $(3.6 \mathrm{~cm}$ long, $2.3 \mathrm{~cm}$ in diameter) were produced at $7 \mathrm{~W}$ (for 9 minutes, $3780 \mathrm{~J}$ ). However, pilot in vivo experiments in pigs with 4 fibres and the combination of 7 W for 540 sec. rapidly led to massive carbonisation in the central area and destruction of the 4 fibres; this could be related to the higher temperature of the surrounding tissue.

Results of ex vivo experiments are of value only if they can be extrapolated to the $i n$ vivo situation. By performing the ex vivo experiments at $28^{\circ} \mathrm{C}$, the optimal distance between the fibres might have been slightly underestimated. It should also be noted that the optical properties of normal liver tissue are not identical to human liver metastases as shown by Germer et al. (1998). As described in chapter 7, experiments with ILC in human hepatic metastases, show areas of coagulation of tumour and surrounding liver tissue similar to those found in this study. In addition, blood perfusion of the target tissue influences the magnitude of the destructive effect by cooling (Svaasand et al, 1985; Whelan et al, 1995). As will be demonstrated in pigs in chapter 5, occlusion of the portal inflow during laser application increases the volume of coagulation five fold. For this reason we and others (Tranberg et al, 1996; Germer et al, 1997; Moller et al, 1997a) occlude the vascular inflow during ILC. As the hepatic inflow is subsequently limited to the artery, which is relatively irrelevant to perfusion of the hepatic parenchyma, no cooling effect is exerted. Therefore, we do not expect to have to adapt the findings, presented here, for the effects of blood perfusion in vivo.

The optimal mutual fibre distance determines the size of coagulation in the plane perpendicular to the fibres. The size of coagulation in the plane parallel to the fibre, is mainly determined by the length of the cylindrical diffusing tip. In this study, fibres with a diffusing tip of $2 \mathrm{~cm}$ length were used. Larger or smaller lesions can be produced by adjusting the length of the fibre tip. Likewise lesion shape can be adapted to tumour geometry.

Two beamsplitters for Nd:YAG light have been described in more detail with regard to the used mutual fibre distance. In a study in dogs, Steger et al. (1992a) differentiated between $1 \mathrm{~cm}$ and 1.5 cm mutual fibre distance between 4 bare-tip fibres illuminated simultaneously with Nd:YAG light. Largest lesions were produced at $1.5 \mathrm{~cm}$. A direct comparison, however, between their study and the present results is hampered by the different conditions and the difference in applied laser power. 48 
Steger et al. were limited to $1.5 \mathrm{~W}$ per fibre due to high energy losses in the used beamsplitter. Ivarsson et al. (1998) studied the use of simultaneous application of four sapphire spherical distributing fibres in ex vivo bovine liver with Diode Laser light $(\lambda=805 \mathrm{~nm})$. The used mutual distance between the fibres of $2 \mathrm{~cm}$ was chosen on the basis of pilot experiments and was not varied in the described experiments. Another important feature of their study was the use of a thermometry system with microprocessor controlled feedback to the laser. Used duration of Diode light up to 1.8 $W$ was 40 minutes compared to $7 \mathrm{~W}$ for maximal 4 minutes in the present study. The difference in used laser parameters is reflected in the largest volumes produced in both studies. In Ivarssons' study, maximal volume of coagulation was $2.7 \mathrm{~cm}^{3}$, compared to $44.5 \mathrm{~cm}^{3}$ in our experiments.

In conclusion, we showed that by using a $1 \times 4$ beamsplitting device the maximal volume of coagulation produced with ILC can be substantially increased. The described construction results in a high transmission efficiency for high power Nd:YAG light with minimal variation between the outgoing beams. For a maximal profit of simultaneous multiple fibre application the optimal combination of power and distance between the fibres needs to be assessed. In this model lesions with a largest diameter of $5 \mathrm{~cm}$ were produced. 


\section{,}




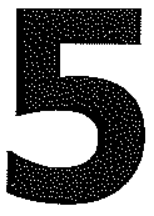

\section{The importance of eliminating portal flow in producing large intra-hepatic lesions with Interstitial laser coagulation}

J Heisterkamp, R van Hillegersberg, PGH Mulder, EL Sinofsky \& JNM IJzermans. British Journal of Surgery 1997, 84; $1245-1248$.

\subsection{INTRODUCTION}

In the previous two chapters strategies to increase the volume of coagulation were investigated in $\mathrm{ex}$ vivo porcine liver. Interstitial laser coagulation (ILC) with four fibres with a diffusing-tip applied simultaneously resulted with optimal mutual fibre distance in lesions of $5 \mathrm{~cm}$ in diameter. In these experiments perfusion of the organ was not taken into account.

Various previous studies have shown preservation of viable cells surrounding larger vessels after ILC. These cells probably survive as the result of the cooling effect provided by the blood flow (Matthewson et al, 1987; LaHaye et al, 1995; Whelan et al, 1995). Furthemore, computer simulations (Roggan and Muller, 1994) and mathematical modelling (Whelan et al, 1995) predict a four- to five- fold increase of the area of coagulation in liver without blood flow. However, the relatively small size of previous animal models and the small volume of coagulation that could be produced may have obscured such an effect. The puppose of the present study was to investigate the effects of the optimized treatment parameters of the ex vivo experiments and the influence of hepatic blood flow, in vivo. Lesion size and configuration were assessed after ILC in a porcine model with and without clamping the large hepatic vessels.

\subsection{MATERIALS \& METHODS}

Experimental groups and laser treatment

Laser treatment was carried out with either physiological flow through the liver ( $n=9$, flow' condition), or with clamping of the portal vein $(n=8$, partial flow condition) or with clamping of both portal vein and hepatic artery $(n=6$, non flow' condition). The vessels were clamped just before insertion of the 
introduction cannulas. Flow was restored immediately after laser coagulation; flow occlusion never exceeded 15 minutes. The experiments, described in chapters 3 and 4, in ex vivo liver had shown an optimal synergistic effect between the four fibres when placed $2 \mathrm{~cm}$ apart and illuminated with $7 \mathrm{~W}$ for 258 seconds. Pilot studies in pigs showed massive carbonisation with these parameters, therefore the combination of $5 \mathrm{~W}$ for 6 minutes was used.

\section{Animals and anaesthesia}

Ten female pigs of pure Yorkshire breed $(75-90 \mathrm{~kg}$, age $8-12$ months) received standard diet; on the experimental day they were kept fasted. The animals were anaesthetised with azaperon $(\mathrm{im}, 0.05 \mathrm{ml} / \mathrm{kg}$ ) and after administering ketamine (im, $10 \mathrm{mg} / \mathrm{kg}$ ) and thiopental (iv, $10 \mathrm{mg} / \mathrm{kg}$ ) they were intubated and ventilated artificially with a mixture of nitrous oxide:oxygen (1:2) and $1.5 \%$ isoflurane. During ventilation, anaesthesia was maintained with pancuronium (iv, $4 \mathrm{mg}$ ) and fentanyl (iv, $0.1 \mathrm{mg}$ ). After the experimental procedures the pigs were sacrificed without recovery from the anaesthesia with a bolus injection of pentobarbifal (iv). The experimental protocol adhered to rules laid down in the Dutch Animal Experimentation Act and was approved by the Committee on Animal Research of the Erasmus University.

\section{Laser equipment}

A Nd:YAG laser [ $\lambda=1064 \mathrm{~nm}, \mathrm{MBB} 4060 \mathrm{~N}$, Domier, München, Germany] was connected to an optical beamsplitting device [Lightstic $4 \mathrm{X}^{\mathrm{TM}}$, Cardiofocus, West-Yarmouth, Ma, USA], supplying 4 fibres with cylindrical diffusing tips of $2 \mathrm{~cm}$ length [Ø1.65 $\mathrm{mm}$, Lightstic ${ }^{\mathrm{TM}}$, Cardiofocus].

\section{Experimental procedures}

Surgical procedures were performed in a fully equipped animal operating theatre, using standard sterile surgical techniques. For optimal exposure of the different liver lobes a thoracolaparotomy was performed. The liver was mobilised and the structures in the hepatodurodenal ligament were identified. To ensure reproducible fibre insertion a custom built Perspex template with 4 openings at a mutual distance of $2 \mathrm{~cm}$ was used. After positioning this template on the liver, 4 intravenous canmulas [14G Adsyte, Becton-Dickinson, Madrid, Spain] were inserted and the needles withdrawn. The laser fibres were put through the cannulas, which were then redrawn entirely out of the liver (Figure 5.1). After laser application the liver capsule was marked with diathermy to allow identification of the respective 
lesions. Immediately after the experiment the animals were killed and the liver was removed. The coagulated lesions were sliced perpendicularly to the plane of fibre insertion. The presence of fusion of the coagulated zones surrounding the fibres was noted. The extent of the lesion was determined, using the macroscopically detectable whitish colour change of the coagulated liver tissue. Height ( $h$, plane along fibre insertion) and width ( $w$, largest plane perpendicularly to fibre insertion) of the lesions were measured using callipers. The volume was then calculated using the formula $h \times w^{2} \times \pi / 6$ (Carlsson et al, 1983). When separate coagulative zones surrounding the four fibres were present, the separate calculated volumes were summed to obtain the total coagulated volume.

Tissue was fixed in 3.6\% formaldehyde, dehydrated in graded ethanol solutions and xylene and then embedded in paraftin. Sections $5 \mu \mathrm{m}$ thick, of the entire plane of dissection were cut for light microscopy and then stained with haematoxylin and eosin.

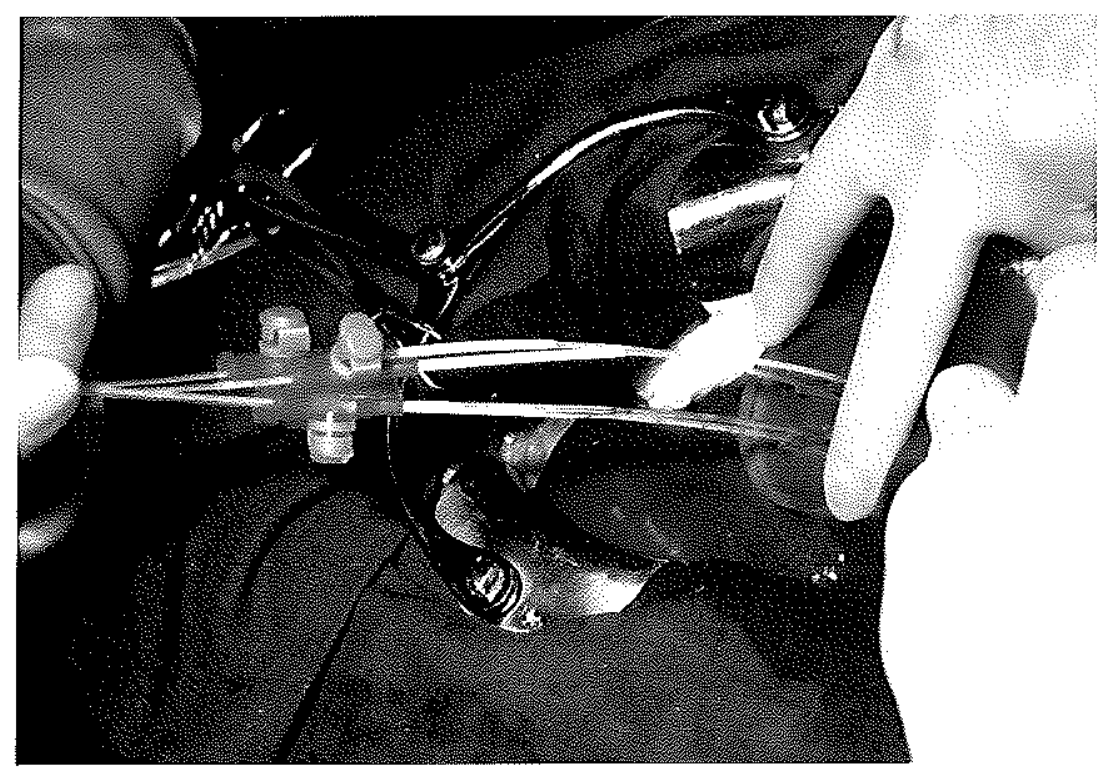

Figure 5.1 Intraoperative view with the Perspex template, insertion cannulas and laser fibers in situ at $2 \mathrm{~cm}$ mutual distance, prior to laser treatment in a peripheral location in pig liver, approached with a small upper midline laparotomy. During laser treatment ( $5 \mathrm{~W}$ per fiber for 6 minutes), portal flow was occluded with a vesselloop in the hepatoduodenal ligament (not visible). 


\section{Statistics}

For each flow condition, mean volume and range were calculated across the number of lesions. Values are expressed as mean (range). Raw data were analysed using an unbalanced repeated measures ANOVA model with flow as independent variable and volume as dependent, regarding the pig as experimental unit. To test for differences in volume by flow condition the Wald test was used. To test for the dependency of fusion (yes/no) on flow, an exact trend test was used in $2 \times 3$ contingency tables, stratified by experimental animal. Results were considered statistically significant at $P$-values less than 0.05 .

\subsection{RESULTS}

Thirty-one lesions were produced, resulting in 23 evaluable lesions in 9 pigs. One pig died before laser treatment, due to anaesthetic complications. Five lesions were not included in the evaluation because of doubt on laser output, due to technical malfunction. In 3 experiments, inadequate withdrawing of the cannulas resulted in melting and massive carbonisation. This problem was resolved by changing from black to transparent cannulas.

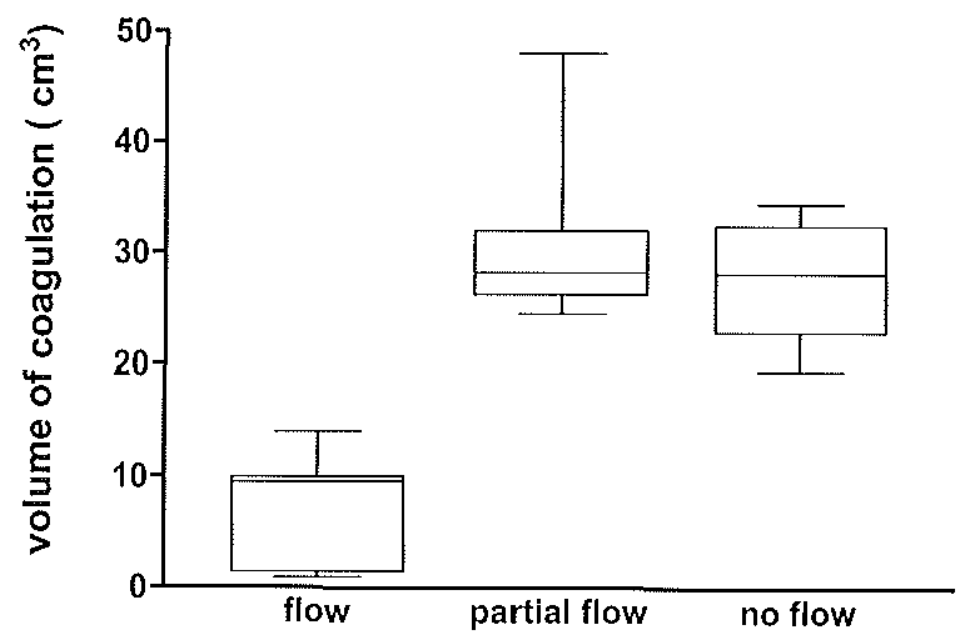

Figure 5.2 Box whisker plot showing the range of volumes of the lesions produced under the three flow conditions. The box extends from the 25 th percentile to the 75 th percentile, with a horizontal line at the median, Volumes were calculated using the formula of the rotational ellipsoid (Carlsson et al, 1983) with the dimensions of the whitish coagulated area. 


\section{Macroseopy}

A circular well defined zone of coagulated tissue was found surrounding the insertion sites of the four fibres. Under the flow condition four separate circular zones could be identified (Figure 5.3). Under the partial flow and non flow condition however, fusion of the coagulated zones between the fibres always occurred. This coagulated zone under the partial and non flow condition was encircled by a haemorrhagic rim (Figure 5.3). Table 5.1 and figure 5.2 summarise the results. The coagulated volume with flow $\left[6.5 \mathrm{~cm}^{3}(1.1-12.6)\right]$ was significantly smalter than with partial flow' $\left[30.6 \mathrm{~cm}^{3}(24.6-48.6)\right]$ and with non flow $\left[26.6 \mathrm{~cm}^{3}(19.5-34.5)\right],(P<0.001)$. No significant difference was found between the coagulated volumes under the partial versus the non flow condition, $(P=0.545)$. Significantly more fusion of the lesions was found under the partial and non flow condition compared to the flow condition $(P=0.0053)$.

\section{Microscopy}

In the area of coagulation, 3 zones could be distinguished (Figure 5.4). Directly adjacent the fibre tracks, elongated spindle-shaped cells were visible, surrounded by a broad area, containing dilated sinusoids and hepatocytes with vacuolated cytoplasm. The outer, macroscopically detectable, haemorrhagic zone consisted of hepatocytes with less vacuolated cytoplasm and hyperaemic sinusoids. This zone was also present in lesions created under the flow condition, although not macroscopically visible. Hepatocytes outside this area had a nomal appearance, however with dilated sinusoids.

Table 5.1. Characteristics of lesions produced by interstitial laser coagulation in the liver, with 4 fibres applied simultaneously ( $5 \mathrm{~W}$ per fibre/ $6 \mathrm{~min}$ ).

\begin{tabular}{|c|c|c|c|}
\hline & $\begin{array}{l}\text { flow } \\
n=9\end{array}$ & $\begin{array}{c}\text { partial flow * } \\
n=8\end{array}$ & $\begin{array}{c}\text { non flow' } t \\
n=6\end{array}$ \\
\hline fusion $(n)$ & 0 & 8 & 6 \\
\hline height $(\mathrm{cm})+$ & $2.2(1.6-3.1)$ & $2.9(3.0-4.0)$ & $3.0(2.9-3.2)$ \\
\hline width $(\mathrm{cm}) \frac{t}{t}$ & $1.2(0.5-2.1)$ & $4.5(4.3-5.1)$ & $4.1(4.0-4.5)$ \\
\hline volume $\left(\mathrm{cm}^{3}\right) \pitchfork S$ & $6.4(1.1-12.6)$ & $30.6(24.6-48.6)$ & $26.6(19.5-34.5)$ \\
\hline
\end{tabular}

Notes: * Portal vein occluded, $\uparrow$ Portal vein and hepatic artery occluded, $\dot{+}$ mean (range), $\S$ The volumes of non-fused lesions were summed. 


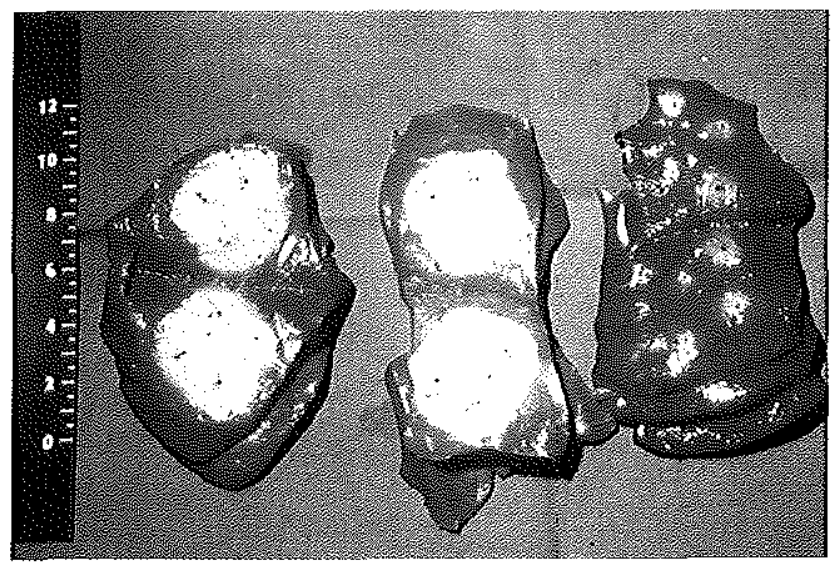

Figure 5.3 Macroscopic view of lesions produced under the three experimental flow conditions, showing the largest sectioned planes perpendicular to fibre insertion. Left: With both hepatic artery and portal vein clamped (non flow), Middle: with portal vein clamped (partial flow) and right: with physiological flow through the liver (flow). All laser applications were performed with 4 fibres simultaneously using $5 \mathrm{~W}$ per fibre for 6 minutes.

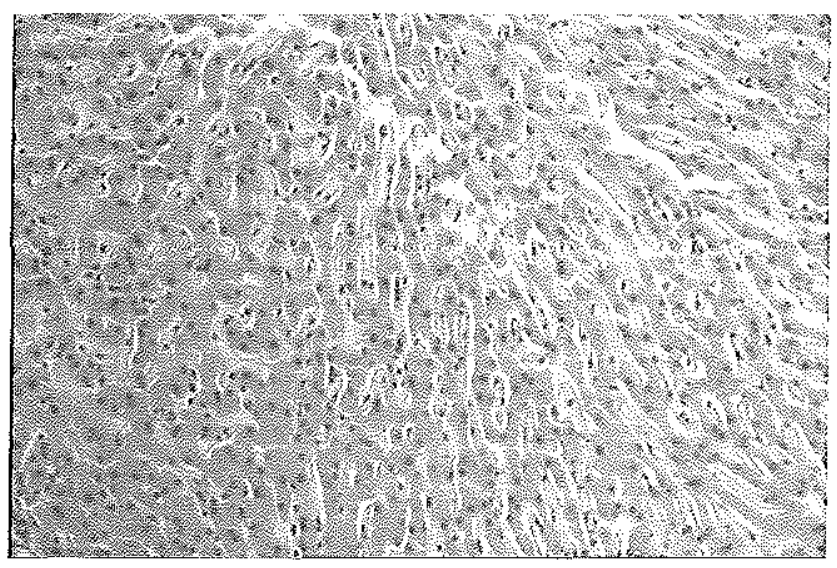

Figure 5.4 Microscopy of the middle zone of coagulation after ILC with portal inflow occlusion. Hepatocytes are visible with less vacuolated cytoplasm within areas with dilated sinusoids (H\&E, magnification $\times 300$ ). 


\subsection{DISCUSSION}

In this study the influence of hepatic blood flow on the coagulated volumes produced by ILC in the liver was investigated. Occlusion of the portal flow substantially increased the area of thermal necrosis. In this way intra-hepatic lesions up to $5.1 \mathrm{~cm}$ in diameter could be produced in an in vivo porcine model.

In these experiments, the lesion size under the flow condition was only 20 per cent of the lesion size produced under the partial flow and non flow condition. Recent computer simulations by Roggan et al. are consistent with the experimental results presented here (1993). They calculated that the coagulation area created with one fibre in a perfused liver would be only 10 per cent of the coagulation area in a non-perfused liver. Tranberg et al. used clamping of both hepatic artery and portal flow in their series of 12 patients treated at laparotomy, based on the supposition that hepatic perfusion would have a lesion reducing effect (1996). This study provides a rationale for their assumption.

Under the partial flow and non flow' condition, a synergistic effect between the four fibres occurred, leading to fused coagulated lesions of $30 \mathrm{~cm}^{3}$. This result is comparable to that found in the ex vivo situation (Svaasand et al, 1985). The synergism between the four simultaneously applied fibres is probably caused by the reduced heat dissipation in the area between the fibres. Under the flow condition, however, fusion never occurred and four scparate, symmetrical, zones of coagulation were found. Furthermore, the zones surrounding each fibre were smaller than in the ex vivo situation. The explanation for this lesion reduction by hepatic blood flow is probably twofold. Firstly, the Nd:YAG light is absorbed by the haem in the erythrocytes (Henkel et $a l, 1996$ ) and secondly, the produced heat is transferred through the vascular system as a result of the hepatic blood flow (Leen et al, 1993; Whelan et al, 1995). Because of the symmetry of the four separate zones produced under the flow condition, we propose that the capillary perfusion rather than the flow in a nearby large vessel was responsible for the lesion size reduction. Moreover, selective preservation of tissue surrounding large vessels was not found in this study.

In contrast to the findings in this sludy, Steger et al. (1992a) reported fused lesions with hepatic flow in canine liver. This observation may be explained by the smaller mutual fibre distance used in their study (1.5 $\mathrm{cm}$ compared to $2 \mathrm{~cm}$ ), causing overlap of the lesions produced by each fibre. In addition the amount of cooling by the blood flow in the smaller canine liver is much less than in the porcine liver. The $2 \mathrm{~cm}$ distance between the fibres in this study was chosen on the basis of the results in ex vivo liver described in chapter 4 . 
No difference was found between clamping of both portal vein and hepatic artery or portal vein alone. These results express the large difference in arterial and portal flow in both a nomal and a metastases bearing liver (Leen et al, 1993). Although it is generally assumed that hepatic metastases are mainly supplied by the arterial branch (Brecdis and Young, 1954), we do not expect the necessity to clamp both hepatic artery and portal vein in the clinical situation. The poorly organised vascular bed in the tumour will probably cause only minimal tissue cooling (Strohmeyer et al, 1987; Gocbel, 1994). Particularly in the often ischaemic centre of the tumour little extra effect can be expected from eliminating the portal flow. The fumour, however, is surrounded by very well perfused liver parenchyma, in which the cooling effect is exerted by the portal flow, as found in this stady. Therefore, eliminating portal flow should enable the destruction of the vital rim of the tumour and a margin of normal liver tissue.

As the net differences in optical properties between liver and tumour arc negligible (van Hillegersberg et al, 1993b), no differences are to be expected in the magnitude of the thermal effect between these two. Indeed in chapter 8 it is found that ILC in human hepatic metastases results in areas of coagulation of tumour and surrounding liver tissue similar to this study.

The microscopic findings are in concordance with previous studies (Bosman et al, 1991; Steger et al, 1992a; van Hillegersberg et al, 1993a). Thomsen also described the peripheral haemorrhagic zone surrounding the coagulated tissue (1991). As even outside this zone dilated sinusoids were found, we expect that the cells in this haemorrhagic rim will not survive. Further studies on this topic are described in chapter 7.

The procedure described here requires a laparotomy. With advancing radiological and laparoscopical skills it will eventually be possible to perform the entire laser treatment, including occlusion of the portal vein, percutaneously. This will require reliable detection of intrahepatic tumours, combined with on-line monitoring of the induced tissue coagulation. Studies to the latter aspect are described in the next part of this thesis. 
PART III LASER-TISSUE INTERACTION AND MONITORING 


\section{Interstitial laser coagulation in porcine liver with portal inflow occlusion: Central versus peripheral lesions at long term}

J Heisterkamp, R van Hillegersberg, PE Zondervan \& JNM IJzermans. Journal of Vascular and Interventional Radiology 1999, 10; 825-831

\subsection{INTRODUCTION}

Interstitial laser coagulation (ILC) is one of the modalities aiming at local tumour destruction with minimal associated parenchymal damage and promising results have been demonstrated in animal experiments and clinical case-scries (Matthewson et al, 1987; Amin et al, 1993c; van Hillegersberg et al, 1994; Vogl et al, 1997). Several novel modifications to the technique allow the destruction of volumes with a diameter of $5 \mathrm{~cm}$ in ex vivo tissue. In chapter 5 we described that portal inflow occlusion is a prerequisite to obtain these results in in vivo conditions. With physiologic flow through the liver, the volume of coagulation is reduced to $10-20 \%$ and tissue is preserved surrounding large vessels (Isbert et al, 1997; Orth et al, 1997). This is in accordance with theoretical and experimental data, showing that large vessels act as heat sinks and would be spared from coagulation (Matthewson et al, 1987; Whelan et al, 1995). As with portal inflow occlusion the protective capacity is lost, the question arises whether this technique would be safe when coagulation is induced in the vicinity of large portal or hepatic veins. The purpose of the present study was therefore to investigate the systemic and local effects at long-term of $\amalg C$ with portal inflow occlusion in a porcine model. The effects on the major vascular and ductal structures in the centre of the liver were assesscd relative to the effects in the periphery of the liver.

\subsection{MATERIALS AND METHODS}

\section{Experimental groups}

There were 2 experimental groups of 8 pigs each. The animals were randomly allocated to the two experimental groups preoperatively (6.1). In each animal one laser application was performed. In one 
group, laser fibres were positioned in the hilar tissue cranially in the left central lobe (referred to as central group). In the other group, the fibres were positioned in the margin of the left central lobe (referred to as peripheral group). Of each group, the first 4 operated animals were sacrificed at 3 months postoperative, the next 2 after 2 months and the last 2 pigs at 1 month after the experimental procedure.

\section{Animals and anaesthesia}

Sixteen adult female pigs of a crossbreed of the Danish Landrace and Yorkshire pigs $( \pm 50 \mathrm{~kg}$ ) received standard diet; the day before and at the day of operation they were kept fasted. Prior to surgery a depot dose of penicillin was injected subcutaneously. The animals were anaesthetised with azaperon (im, 0.05 $\mathrm{ml} / \mathrm{kg}$ ) and after administering ketamine (im, $10 \mathrm{mg} / \mathrm{kg}$ ) and thiopental (iv, $10 \mathrm{mg} / \mathrm{kg}$ ) they were intubated and ventilated artificially with a mixture of nitrous oxide:oxygen $(1: 2, \mathrm{~V}: \mathrm{V})$ and $1.5 \%$ isoflurane. During ventilation, anaesthesia was maintained with pancuronium (iv, $4 \mathrm{mg}$ ) and fentanyl (iv, $0.1 \mathrm{mg}$ ). Normal saline, $1000 \mathrm{ml}$, was given intravenously over the procedure. After the experimental procedures, the effects of pavulon were antagonised with neostigmine (iv, $2 \mathrm{ml}$ ) and the pigs were hand ventilated until independent breathing was restored (always $<5 \mathrm{~min}$.). Duration of artificial ventilation never exceeded $1.5 \mathrm{hr}$. After the follow up period animals were sacrificed with a bolus injection of pentobarbital (iv). The experimental protocol adhered to rules laid down in the Dutch Animal Experimentation Act and was approved by the Committee on Animal Research of the Erasmus University Rotterdam.

\section{Laser equipment}

A Nd:YAG laser $[\lambda=1064 \mathrm{~nm}$, Laserscope, KTP/YAG 832, San José CA, USA] was connected to an optical beamsplitting device [4X Lightstic, Cardiofocus, West-Yarmouth MA, USA], supplying simultaneously 4 fibres with cylindrical diffusing tips of $2 \mathrm{~cm}$ length and an outer diameter of $1.65 \mathrm{~mm}$ [ightstic ${ }^{\mathrm{n} 1}$, Cardiofocus]. Characteristics and tissue effects in ex vivo tissue of the laser fibres and the optical beamsplitter have been described in chapters 3 and 4 . 


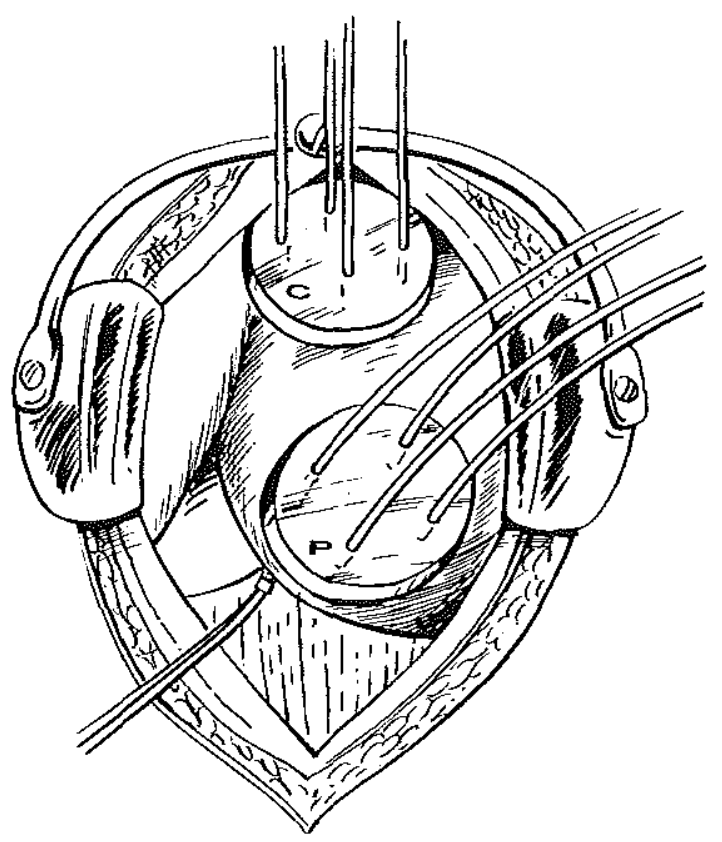

Figure 6.1 The two different locations for $\mathrm{ILC}$ in the respective experimental groups (both groups consisted of 8 pigs) with template, insertion cannulas and laser fibres in situ. The left central lobe of the liver is seen at its parietal surface (facies ventralis) with the right central lobe just visible. In group 1 ( $C$, central group) the 4 laser fibres were guided through insertion cannulas which had been inserted from cranially into the hilar tissue and in the second group ( $P$, peripheral group) the 4 laser fibres were guided through insertion cannulas which were at a peripheral location in the same lobe. In each animal 1 lesion was produced. The vessel loop was used to occlude portal flow in the hepatoduodenal ligament (not visible) during laser treatment.

\section{Experimental procedures}

Surgical procedures were performed in a fully equipped animal operating theatre, using standard sterile surgical techniques. A small upper midline laparotomy was performed which allowed, after dissecting the falciform and round ligament, access to the parietal aspect of the left central liver lobe and to the hepatoduodenal ligament. The portal vein was identified and isolated in the hepatoduodenal ligament and controlled with a vessel loop. Just prior to inserting the introduction camulas into liver, the vessel loop was snared for portal inflow occlusion. A custom built Perspex template with 4 openings at a mutual distance of $2 \mathrm{~cm}$ was used for fibre positioning. After positioning this template on the liver at 
the randomised location, 4 intravenous cannulas [14G Adsyte, Becton-Dickinson, Madrid, Spain] were inserted and the needles withdrawn. The laser fibres were put through the cannulas, which were then redrawn entirely out of the liver (Figure 5.1). Laser application was performed with $5 \mathrm{~W}$ per fibre during 6 minutes, based on the studies in chapters 3-5. In the peripheral group, coagulation in the underlying left lateral lobe was prevented by wet gauzes between the lobes. Portal flow was restored immediately after laser coagulation; flow occlusion never exceeded 10 minutes. Haemostasis at the sites of fibre insertion was performed with manual pressure or when necessary with application of Lyostipt ${ }^{\mathrm{TM}}$ [Braun, Melsungen, Germany]. Extent of coagulation was assessed with bimanual palpation. Subsequently the laparotomy was closed in two layers. The entire procedure never exceeded $1 \mathrm{br}$. Cutaneous sutures were removed 2 weeks after surgery under light sedation with ketamine (im, 5 $\mathrm{mg} / \mathrm{kg}$ )

\section{Evaluation}

Just prior to surgery, two weeks after the surgery and just prior to sacrifice, the animals were weighted and venous blood was sampled from an ear vein. Serum was stored at $-70^{\circ} \mathrm{C}$ till analysis with a commercially available laboratory robot [ELAN analyser, Eppendorf ${ }^{\mathrm{TM}}$, Hamburg, Germany]. Alkaline phosphatase (AP) and $\gamma$-Glutamyl transpeptidase $(\gamma-\mathrm{GT})$ were determined as parameters indicating bile duct obstruction. Serum alanine aminotransferase (ALAT) and aspertate aminotransferase (ASAT) were used as parameters indicating cellular damage. Total bilirubine (tBL) and albumine (Alb) were measured as parameters for liver function. After sacrifice the abdomen and thorax were opened and inspected, blinded for the experimental group involved. Tissue with signs for abnormalities was excised and fixed in 3.5\% formaldehyde. The liver was then removed and the left central lobe was cut in slices of $0.5 \mathrm{~cm}$ from periphery to hilus. When coagulation necrosis was present, height $(h$, suspected plane along fibre insertion) and width (w, largest suspected plane perpendicularly to fibre insertion) were measured with callipers. The volume of coagulation was calculated using the formula $h \times w^{2} \times \pi / 6$ (Carlsson et al, 1983). The entire left central lobe was fixed in 3.5\% formaldehyde. Relevant sections of maximal $6 \mathrm{~cm}$ square and $1 \mathrm{~cm}$ thick were excised, dehydrated in graded ethanol solutions and xylene and embedded in paraffin. Transversal sections, $10 \mu \mathrm{m}$ thick, of the entire plane of dissection were cut for light microscopy and then stained with haematoxylin and eosin (H\&E). 


\section{Statistics}

All numerical data are expressed as mean \pm standard error of the mean (SEM), and tested for difference between the experimental groups with Student's $t$ test. Differences between groups associated with experimental group or length of follow up in serum levels and weight (sacrifice - day of surgery) and volume of coagulation were analysed using a two-way of analysis of variance test with experimental group and length of follow up as factor (Venables and Ripley, 1994). Results were considered statistically significant at $P \leq 0.05$.

\subsection{RESULTS}

Seventeen animals were operated in this study. During laser application in the 11 th animal, 2 fibres carbonised and were destroyed at $2 \mathrm{~min}$. and 1 fibre at 3 min. In this case, laser application was stopped and the animal was excluded from analysis. Lascr application in its substitute (17th animal) was at the same location (peripheral) with same length of follow up ( 2 months) as randomised. In the 3rd animal, 1 fibre tip remained in situ after redrawing the fibre following laser application, without signs of carbonisation or destruction. All animals recovered uneventfully from the operation and no adverse events became apparent during follow up.

At post mortal examination, dense and calcified strings of adhesions, increasing in strength with length of follow up, were found between the laparotomy wound, peritoneum and intra-abdominal structures. With gentle manipulation, the hepatic surface could be freed from the adhesions except for the presumed site of fibre insertion.

\section{Chemistries and weight}

No significant differences between the 2 groups of 8 animals in weight and serum levels were present preoperatively $(P \geq 0.08$, Table I).

Compared to the preoperative values in none of the pigs a statistical change was found at two weeks after the intervention. When analysed for association with either experimental group or length of follow up, no significant differences were found $(P \geq 0.12$, Table 6.1). A significant increase of weight was found with time of follow up $(P<0.01)$ without a significant difference in increase between the 2 experimental groups ( $P=0.73$, Table 6.1). 
Table 6.1. Changes of liver functions (IU/l), preoperative and after follow up, of the pigs, randomised into 2 groups with a different location of interstitial laser treatment ( $n=8$ per group). Data shown as mean (standard error of the mean).

\begin{tabular}{|c|c|c|c|c|}
\hline & \multicolumn{2}{|c|}{ preoperative } & \multicolumn{2}{|c|}{ end of follow up * } \\
\hline & \multicolumn{2}{|c|}{ location of treatment $\dagger$} & \multicolumn{2}{|c|}{ location of treatment $\uparrow$} \\
\hline & central & peripheral & central & peripheral \\
\hline AP & $129.1(9.4)$ & $141.0(7.0)$ & $109.0(9.6)$ & $125.6(10.3)$ \\
\hline$y-\mathrm{GT}$ & $26.3(11.7)$ & $37.7(3.9)$ & $31.5(13.4)$ & $44.9(4.7)$ \\
\hline ALAT & $37.1(2.6)$ & $43.3(1.7)$ & $35.9(1.9)$ & $42.3(2.6)$ \\
\hline ASAT & $33.5(3.0)$ & $36.9(4.2)$ & $49.0(12.8)$ & $47.9(5.1)$ \\
\hline Alb & $32.8(1.4)$ & $32.3(1.2)$ & $35.9(0.6)$ & $36.2(0.5)$ \\
\hline tBIL & $3.3(2.2)$ & $4.2(0.9)$ & $3.0(1.7)$ & $2.6(0.1)$ \\
\hline
\end{tabular}

Notes: * Values irrespective of length of follow up (for 1,2 or 3 months), $\dagger$ No significant differences between the 2 experimental groups were found preoperatively (Student's 2-tailed $t$ test) and no experimental group related differences were found at end of follow up (2-way analysis of variance with location of treatment (central or peripheral) and length of follow up (1,2 or 3 months) as factors).

\section{Macroscopy}

No atrophy or cholestasis in the lobes treated was found at necropsy; no signs of portal hypertension, hepatic thrombosis or lung emboli were seen.

In general, all lesions showed the same aspect: a round pale area consisting of fixed coagulated tissue encased in a capsule of approximately $\&$ millimetre width. At one month post-treatment, in both of the two pigs with a peripheral lesion and in one of two with a central lesion a small collection of bile ( $<5 \mathrm{~mm}$ in diameter) was found, located near the inner border of the capsule. At 3 months no rests of coagulation necrosis were found in two of four pigs; both in the central and in the peripheral group. In one of these animals (3rd, ceniral group) the fibre tip, which had remained in situ after laser application, was found surrounded by regenerated nomal hepatic parenchyma. In all other pigs a lesion was palpable and found on dissection. Volume of the lesion was largest at 1 month and was smaller with time of follow up $(P=0.02$, Figure 6.2). At any moment volumes were larger in the peripheral group $(P=0.03$ ) with no significant difference in the decrease between the experimental groups $(P=0.10)$. In one case ( 2 months follow up), colliquative necrosis was found in the area of coagulation necrosis; bacteriological analysis did not show infection. 


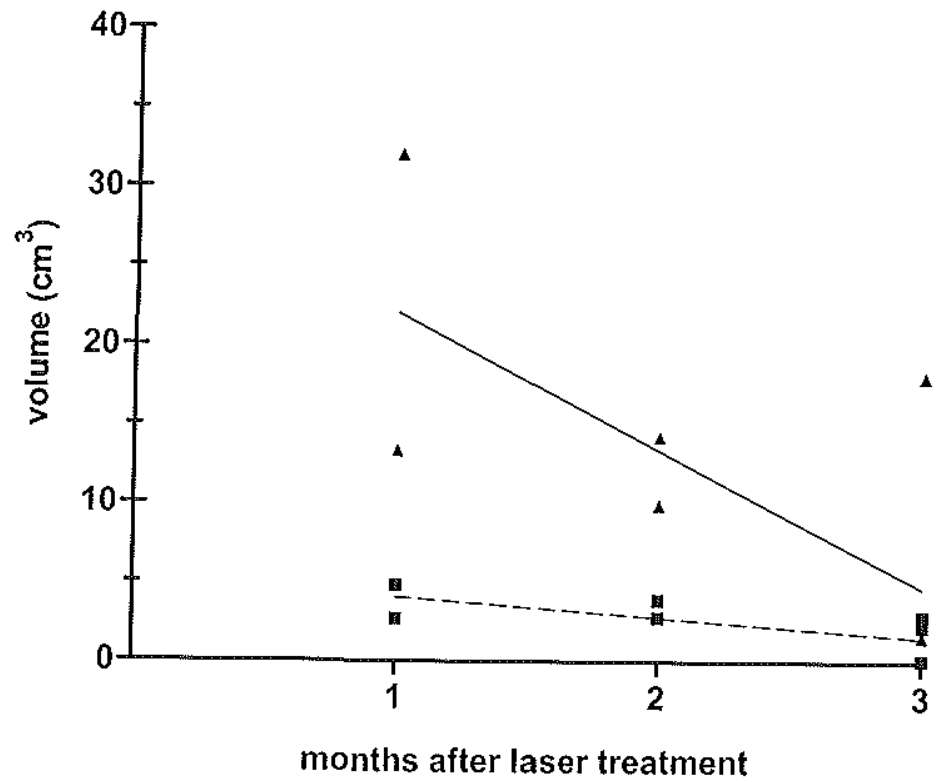

Figure 6.2 Individual volumes of the laser induced coagulation necrosis as found at dissection of the liver after 1, 2 or 3 months post ILC. Laser treatment was with 4 fibres, applied simultaneously (5W per fibre during $6 \mathrm{~min}$ ) with portal inflow occlusion during treatment. Individual volumes are categorised for location of laser treatment: central ( $)$ or peripheral $(\mathbf{A})$, determined by randomisation. Lines indicate the linear regression equation for both experimental groups. Note that at 3 months after laser treatment no rests of coagulation were found in $2 / 4$ pigs in the central group and $1 / 4$ pigs with a peripheral lesion

\section{Microscopy of induced lesions}

A similar histological pattern could be observed in all specimens of laser induced lesions, regardless of the experimental group or diameter of the lesion (Figure 6.3). A very discrete demarcation between hepatic parenchyma, fibrotic rim, and area of coagulation necrosis was present. No abnormalities were seen in the hepatic parenchyma. The fibrotic rim showed extensive mesenchymal reorganisation with a dense network of collagen and reticular fibres in which abundant bile duct regeneration was present from 1 month after treatment. Thrombosis and intimal hyperplasia was seen in small calibre portal branches. Near the area of coagulation necrosis, calcifications were present in the rim. In the transition zone between rim and area of coagulation a few infiltrating cells were present, extending into both zones. In the area of coagulation, the typical 
hepatic architecture had remained intact with loss of intercellular cohesion resulting in oedema filled spaces between the cells. Two cell groups could be discriminated: 1. pale cells without a nucleus and lacking an outer membrane and 2. cells with a shrunken pyknotic nucleus, also lacking a cellular membrane. The eosinophilic cytoplasm was of an intermediate aspect between that of hepatic parenchyma and the anuclear cells. Both types were distributed in clusters throughout the area of coagulation without a clear pattern. Within the coagulum bile ducts had vanished whereas some remaining of former vessels could be seen. Large vessels, portal, arterial and venous, adjacent to the lesions, were always intact both on gross section and at microscopy.

\subsection{DISCUSSION}

In this experimental study the effects of ILC in the liver with portal inflow occlusion were investigated with emphasis on the possible damage of $\Pi \mathrm{LC}$ to adjacent large vessels or bile ducts. No adverse events were found, even in the vicinity of large vessels. A central location in the liver was compared with a peripheral location as most of the tumours eligible for laser treatment will be those near large portal or hepatic veins, causing surgical irresectability.

Only portal inflow occlusion was applied in this study to increase the capacity, based on the results described in chapter 5 in which no difference in the magnitude of coagulation was found between portal occlusion only and portal with arterial occlusion during ILC. The increase of coagulated volume by inflow occlusion is explained by a reduction of heat convection.

Inspection of the specimens retrieved at necropsy confirmed that lesions in the central group had always been produced adjoining large portal or hepatic vessels. Abundant bile duct regeneration was found surrounding the zone of coagulation, indicating hepatocyte regeneration of bile ducts (Shah and Gerber, 1990): probably as the result of damage to medium size bile structures at the segmental level. Possible major damage was assessed by monitoring weight increase and chemical liver functions. Further imaging studies during the follow up were not a priori planned. In none of the pigs the clinical course necessitated investigations such as Doppler ultrasound or cholangiography. Although damage to medium sized bile ducts and larger bile ducts was not clinically significant, it remains to be determined whether ILC of tumors located in the hilar part of the liver can be performed without damaging the major biliary structures, as has been described for cryosurgery for hepatic tumors (Lec et al, 1997). This aspect could not be investigated in the pig model as the confluence of the left and right main hepatic ducts into the common hepatic bile duct 68 
is located outside the liver in pigs (Filipponi et al, 1995). Also, no conclusions can be drawn to the safety and efficacy of ILC near the retrohepatic inferior caval vein. Although the vessel wall is not at risk for thermal damage, as the flow will not be occluded, the depth of the heat sink effect in the hepatic parenchyma and adjacent tumor has yet to be investigated.
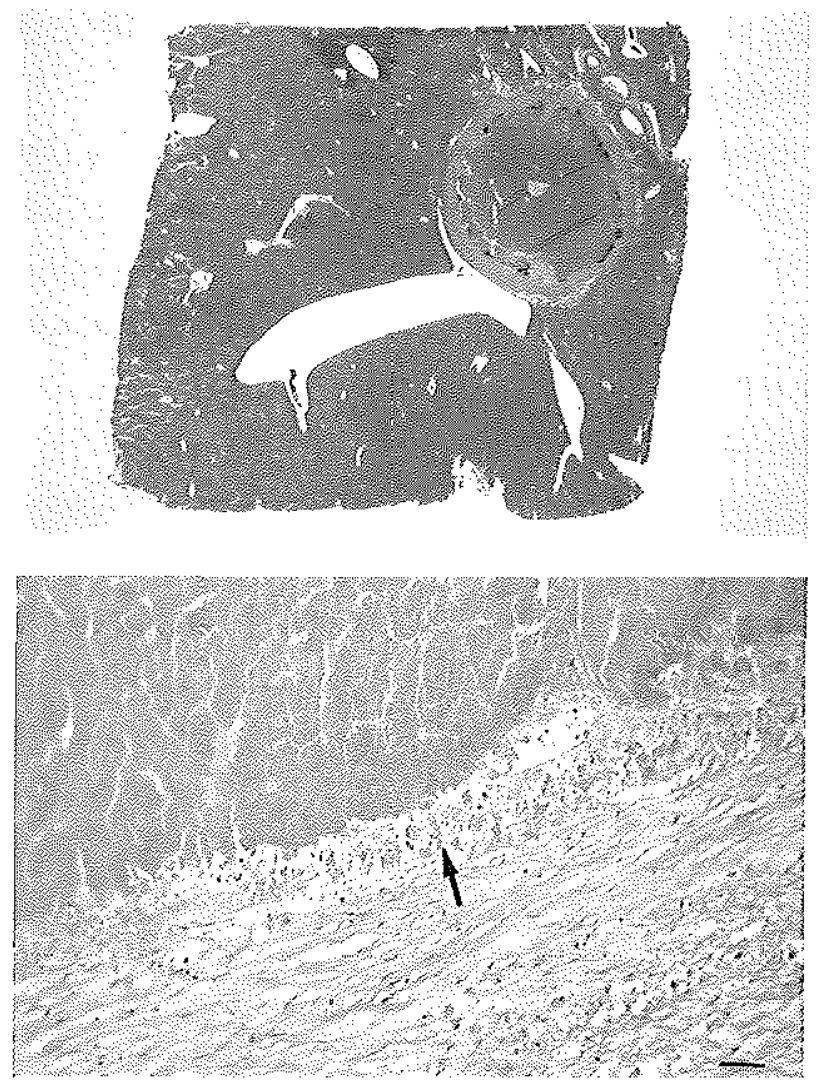

Figure 6.3 Microscopic views of (above) entire dissected plane of area of coagulation necrosis of lesion central in the liver adjacent to the middle hepatic vein $(2.5 \mathrm{~cm}$ in diameter, 1 month after laser treatment) and (under) the fibrotic rim with collagen fibres and calcification, $\Rightarrow$ indicates infiltrating cells into both fibrotic zone and the area of coagulation ( $\times 54$, bar indicates $50 \mu \mathrm{m}$ ). 
In this study coagulation necrosis was found into the adventitia of large vessels, whereas the more inner layers were always intact. A similar effect was found in a study where the coagulation necrosis was induced with cryosurgery (Weber et al, 1997). Several factors may be proposed to protect the essential hepatic structures. The portal branches contain large amounts of collagen which radiates the heat; the arterial input results in flow in the fragile hepatic veins and this arterial inflow protects the arteries and the large biliary ducts from damage. No conclusions can be drawn to the safety and efficiency of $I L C$ near the caval vein. Although the vessel wall is not at risk for thermal damage, as the flow will not be occluded, the depth of the heat sink effect in the hepatic parenchyma and adjacent tumour remains to be investigated.

In this study the local and systemic response of ULC was investigated in healthy porcine liver. In studies with tumour bearing rodents, a comparable encapsulation with the typical coagulation necrosis was found (van Hillegersberg et al, 1994). The histological findings after laser coagulation are in agreement with earlier findings by several investigators in the healthy livers of various animals (Matthewson et al, 1987; Bosman et al, 1991; Steger et al, 1992a; Isbert et al, 1997; Moller et al, 1997a). The absence of cellular lysis after coagulation may explain why no clinically relevant systemic response is found after interstitial laser treatment: as cell walls are not disrupted, there is no release of cellular debris into the circulation. The presence of a nucleus (although pyknotic) in a subgroup of coagulated cells make these cells look vital, illustrating the difficulties in assessing the lethality of ILC. In chapters 7 and 8 further studies are presented.

The regression of the volumes of coagulation is in agreement with other studies where long-term outcome of ILC without inflow occlusion was studied and without attention to the location of treatment in the liver (Bosman et al, 1991; Steger et al, 1992a; Isbert et al, 1997; Moller et al, 1997a). From 1 month post-treatment smaller volumes of coagulation were found in the central group. Probably volumes of induced coagulation in the central group were initially smaller. As central locations are surrounded by more liver parenchyma, the produced heat is convected and distributed over a larger volume, resulting in a smaller volume of coagulated tissue. This emphasises the importance of monitoring the laser-induced effects during treatment. Extrapolation of the regression lines in figure 6.2 suggests an even larger difference just after surgery. Because of the completely surrounding parenchyma, however, central lesions will also be resorbed faster in the inflammatory reaction, adding to the difference in volume of coagulated tissue retrieved at necropsy. Variation in surrounding hepatic tissue, may explain why a greater variance in coagulated volume was found in the peripheral group. In addition the greater arterial flow central in the liver will exert a 70 
cooling effect and add to the difference in coagulated volume. In retrospect we also bold this phenomenon responsible for the considerable variation in coagulated volume assessed just after laser treatment as described in chapter 5 .

In all cases, irrespective of experimental group, dense adhesions were found from 1 month after laser treatment, concentrated between the incision site, the hepatic surface and the hepatoduodenal ligament. We consider the adhesions conspicuously abundant for a minor intervention. Studies on cryosurgery and alcohol injection in a porcine model (Dilley et al, 1993; Vehmas et al, 1993; Brunken et al, 1999) also found dense adhesions between the site of probe insertion and the peritoneum were found. As in this study, adhesions were never a cause of clinically overt problems in the animals. Matching this, pigs are reported to be more prone to adhesion formation than humans (Larsson et al, 1981): extensive adhesions after opening and direct closure of the peritoneum were found in a control group (Attwood et al, 1994) for experiments in which adhesion formation after laparoscopic inguinal hernia repair in pigs was studied. Thus, the adhesions we found may very well be a model dependent finding that will not occur in the human situation.

In conclusion, it was found in this chapter that 1) ILC damage at long term to parenchymal structures is identical at a central location compared to a lesion at the periphery of the liver, 2) portal inflow occlusion can safely be applied in the liver. In addition it was found that the dimensions of produced coagulation are smaller in the central portion of the liver. 


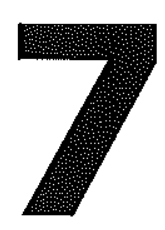

\section{Metabolic activity and DNA integrity in human colorectal liver metastases after Interstitial laser coagulation}

J Heisterkamp, R van Hillegersberg, PE Zondervan \& JNM IJzermans. Submitted for publication

\subsection{INTRODUCTION}

The effects of interstitial laser coagulation (ILC) on tissue have been studied extensively in animal models, both in normal liver tissue as well in implanted tumour models. The response to $\Pi \mathrm{C}$ has been characterised by assessment at several time points following ILC (Schober et al, 1993; Tracz et al, 1993; van Hillegersberg et al, 1994). Morphological characteristics (Dowlatshahi et al, 1992a; Moller et al, 1997b; Prudhomme et al, 1999) and more specifically staining for bromodeoxyuridine (BrdU) incorporation (van Hillegersberg et al, 1994) have nuled out the presence of vital cells from 6 days after ILC. Defining the vitality of the altered cells directly after $\Pi \mathrm{LC}$ is more difficult as morphological observations at microscopy do not allow unambiguous conclusions regarding cellular vitality (Thomsen, 1991). For obvious reasons the follow up after ILC in patients is limited to use of biopsies which are prone to sample bias (Hahl et al, 1990; Dowlatshahi et al, 1992b; Amin et $a l, 1993 \mathrm{c})$. Anecdotally, the entire tumour microscopy could be studied in tissue retrieved at autopsy (Tranberg et al, 1996) at 10 days following ILC.

The investigations described in Part II resulted in an increased lesion size of up to $5.1 \mathrm{~cm}$ in diameter in normal porcine liver. Before implementing these modifications to the traditional ILC technique in a clinical protocol further research is mandatory to answer whether 1) the coagulative effect is sufficient to cause cellular death in tumour and 2) there is a difference in the magnitude of the ILC effect in normal porcine liver versus human tumour. To this end the metabolic activity and DNA integrity was assessed directly after ILC in patients with surgically resected colorectal liver. 


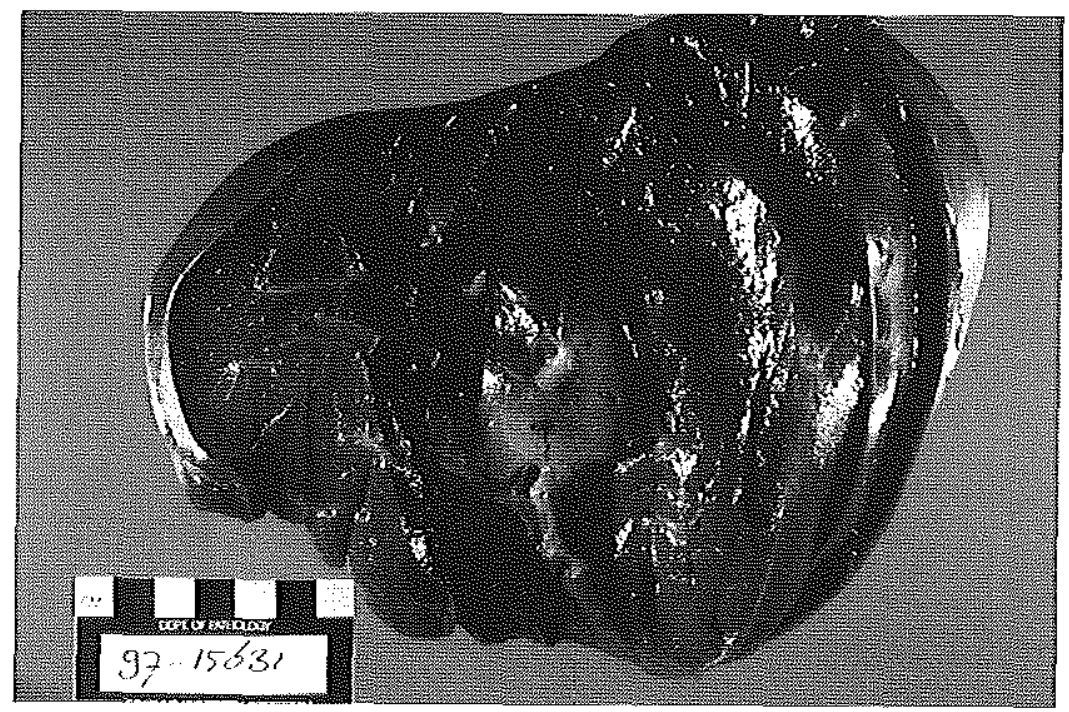

Figure 7.1 Colorectal metastasis in which ILC has been perfomed prior to surgical resection (4 fibres, $5 \mathrm{~W}$ per fibre during 6 minutes). The plane of dissection is perpendicular to plane of fibre insertion. Central the pale tumour ( $2.5 \mathrm{~cm}$ in diameter) which is completely encircled by the whitish coagulation $(4.9 \mathrm{~cm}$ in diameter). Scale in centimeters.

\subsection{MATERIALS AND METHODS}

\section{Resection specimens}

Resection specimens from 10 consecutive liver resections for colorectal metastases were obtained with informed consent from the patient. The specimens contained one or more metastases originating from a previously resected colorectal carcinoma. If multiple metastases were present, the largest tumour only was lased. To be comparable with the experiments in chapter 4 , the first 5 applications were performed in specimens after resection. The specimens were transported immediately following resection in wet guazes to the room where the laser application was performed. Directly after laser treatment the specimen was further processed. To be comparable with the experiments in chapter 5 , laser treatment in the latter 5 applications was performed prior to resection. Liver resection was prepared as usual with transection of the hepatic ligamental attachments and inspection and dissection of the hepatoduodenal ligament. During the routine ultrasound examination, laser fibres were placed intraoperatively in the tumour. The portal flow was occluded in the hepatoduodenal ligament with a vessel loop. After ILC the surgical resection was performed. During resection the hepatic inflow was occluded to minimise blood 
loss from the resection surface. Immediately following resection the specimen was processed. Two specimens from hepatic resections prior to the study period and from which also snap-frozen tissue was available were used as negative controls (colorectal metastases and liver tissue without coagulation).

\section{Laser application}

The number of the used fibres, apllied laser power and duration of exposure to laserpower, were adapted to diameter of the tumour (as assessed with intraoperative ultrasound) based on previous experiments in ex vivo porcine liver (chapters 3-4). Through each fibre, $5 \mathrm{~W}$ was guided during 6 minutes irrespective of the number of fibres. Small bore cannulas (Adsyte 14G, Becton Dickinson, Spain) were introduced transparenchymal into the tumour. By using a custom-built template mutual fibre distance was reproducible. After inserting the fibres through the cannulas, the latter were redrawn and laser application was started.

Nd:YAG laserlight $(\lambda=1064 \mathrm{~nm})$ was guided to fibres with a light diffusing end of $2 \mathrm{~cm}$ (Lightstic ${ }^{\text {TM }}$ Cardiofocus, West Yarmouth, MA, USA, outer diameter $1.65 \mathrm{~mm}$, core diameter $600 \mu \mathrm{m})$. When more than one fibre was used, light was divided with a beamsplitter feeding maximally 4 fibres (as described in chapter 4).

\section{Processing}

A single pathologist reviewed all specimens immediately after resection. Resected specimens were inspected grossly for underlying liver disease, completeness of resection, resection margins and satellite or multiple tumours. The specimen was dissected along the plane parallel and perpendicular to insertion of the fibres. Coagulation was estimated as the macroscopically detectable (whitish) colour change compared to normal coloured liver parenchyma. Height $(h)$ of the lesion and diameter of the largest coagulated plane (w) as well as $h$ and $w$ of the tumour were measured with callipers. Volume was approximated using the volume of a rotational ellipsoid: $V=h \times w^{2} \times \pi / 6$ (Carisson $e$ al, 1983).

Of the resection specimensat least 4 samples were cut at the following locations (Figure 7.2): 1) within zone of macroscopical coagulation and within tumour, 2) within zone of macroscopical coagulation at transition from tumour to hepatic parenchyma, 3) transition from zone of macroscopical coagulation to zone of not coagulated parenchyma and 4) hepatic parenchyma outside zone of macroscopical coagulation. When applicable: S) tumour from a zone ontside zone of macroscopical coagulation or 6 ) tumour from a not lased second tumour. These samples were snap-frozen in liquid nifrogen at $-180^{\circ} \mathrm{C}$ 
for further immunohistochemical examination. Remaining specimen was processed routinely for patient care. The tissue was fixed in $3.6 \%$ formaldehyde, dehydrated in graded ethanol solutions and xylene and then embedded in paraffin.

\section{Staining}

Two parameters were used to assess cell damage: mitochondrial metabolism (NADH histochemistry) and DNA integrity (nick end-labeling).

\section{NADH histochemistry}

Staining with nitro blue tetrazolizm (NBT) can be used to stain NADH-diaphorase activity which is a measure for mitochondrial metabolism. Upon cell death, the activity of NADH-diaphorase subsides (Neumann et al, 1991) and NADH is not metabolised. The snap-frozen samples, stored at $80^{\circ} \mathrm{C}$, were cut in serial sections of $8 \mathrm{~mm}$ with a cryostat. Sections were picked up onto clean glass slides. Incubation solution consisted of $9 \mathrm{mg}$ NADH 3-ml phosphate-buffered-saline; $12.1 \mathrm{ml}$ distilled water and $35 \mathrm{mg}$ NBT [Sigma]. Sections were covered with $100-\mathrm{ml}$ solution and incubated for 60 minutes at $37^{\circ} \mathrm{C}$. Sections were washed with distilled water, mounted in a formaline/macrodex solution medium and covered with a glass cover slip. For negative controls NADH was omitted.

\section{Nick end-labeling (TUNEL)}

In the terminal deoxynucleotidyl transferase (TdT)-mediated dUTP bioton nick end-labeling (TUNEL) method the added DNA polymerase enzyme, terminal deoxynucletidyl transferase (TdT), binds to blund ends of double stranded DNA breaks, thereby identifying DNA fragmentation and thus early stages of necrosis (Gold et al, 1994). Staining was performed as described previously (Gold et al, 1994; Surh and Sprent, 1994). Paraffin embedded tissue was cut in sections $5 \mathrm{~mm}$ thick and placed on DNAse free slides. Subsequently they were dewaxed. After Proteinease K digestion blocking of endogenous peroxydases, sections were washed with TdT buffer ( $\mathrm{pH} 7.2,0.5 \mathrm{M}$ cacodylate, $1 \mathrm{mM} \mathrm{CoCl} 2,0.5 \mathrm{mM}$ DDT, $0.05 \% \mathrm{BSA}, 0.15 \mathrm{M} \mathrm{NaCl}$ ). Incubation time was 2 minutes with $0.5-\mathrm{mM}$ biotin-16-dUTP and 10 U TdT [Boehringer Mannheim, Mannheim, Germany]. After washing in PBS, sections were incubated with streptavidin-biotinylated peroxidase complex specific for Boehringer dUTP. Sections were stained with $\mathrm{DAB}$ and counterstained with Meyers haematoxylin. For negative controls TdT was omitted. 


\section{Evaluation}

Sections were scored qualitatively using light microscopy by an observer unaware of the location in the plane of dissection. With light microscopy $(400 \mathrm{X})$ the cytoplasmatic mitochondrial activity was visible as blue staining. Proportion of staining in 5 different fields of a graticule was scored according to the absence or presence of blue staining cells: If in all 5 fields no activity was scored the NADH-diaphorase was considered to be inactivated in the respective location.

DNA fragments were visible as brownish staining of the nucleus, whereas vital cells stained redgreen. When more than $30 \%$ of the cells observed in 5 grids of the graticule showed nuclear DNA, fragmentation was considered complete; this is twice the largest proportion of positive cells in controls (Kerbel, 1990; Williams et al, 1997). Successful coagulation with cell death was defined of absent NADH-diaphorase activity with disintegrated DNA fragmentation. Sections routinely stained with H\&E were also reviewed and compared for morphological changes.

Table 7.1 Proportion of completed coagulation assessed by NADH and TUNEL staining, in the different locations of interest (see figure 7.2).

\begin{tabular}{ccccccc}
\hline & \multicolumn{7}{c}{ Location } \\
& $\mathbf{1}$ & $\mathbf{2}$ & $\mathbf{3}$ & $\mathbf{4}$ & $\mathbf{5}$ & $\mathbf{6}$ \\
\hline NADH-diaphorase inactivity * & $10 / 10$ & $10 / 10$ & $9 / 10$ & $1 / 10$ & $0 / 3$ & $0 / 6$ \\
DNA fragmentation + & $10 / 10$ & $9 / 10$ & $10 / 10$ & $0 / 10$ & $0 / 3$ & $0 / 6$ \\
\hline
\end{tabular}
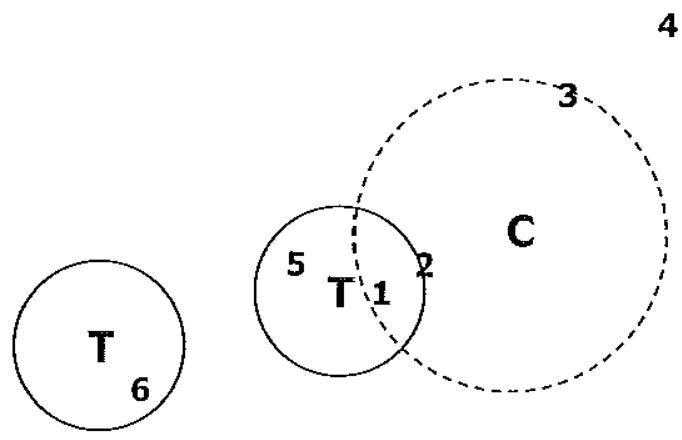

Figure 7.2 Schematic drawing indicating the different locations of interest and sampling (T: tumour, C zone of coagulation). 


\subsection{RESULTS}

In two tumours a single fibre was used, in one tumour 2 fibres and in the 7 remaining, 4 fibres were used with a beamsplitter. In one patient fibre breakdown occurred and the fibre tip remained in the tumour after withdrawing the fibres. This did not affect the subsequent resection.

\section{Macroseopy}

Coagulated tissue, both liver and tumour, was firmer compared to not coagulated tissue. Macroscopic coagulation of liver parenchyma was clearly discemible from native parenchyma (Figure 7.1). There was a sharp transition between the whitish zone coagulation and liver parenchyma without signs of a haemoraghic rim. Charring has not been observed.

Tumour diameter varied between 2.1 and $6.7 \mathrm{~cm}$ and the produced volume of coagulation volume between 2.9 and $5.5 \mathrm{~cm}$. In two tumours the volume of coagulation was smaller than the tumour volume (tumour diameter 6.7 and $4.9 \mathrm{~cm}$ respectively). In another tumour the volume of coagulation was larger than the tumour volume but because of eccentric fibre positioning the induced coagulation comprised a surplus of liver and did not encircle the entire tumour.

The results are summarised in Table 7.2. There were no statistically significant differences in the induced volumes of coagulation between the tumours lased ex vivo or prior to resection $(P=0.48)$. On average the volume of coagulation surpassed the volume of the corresponding tumour with a factor 1.8 (range: $0.34-4.2$ ).

\section{Microscopy}

In the H\&E stained sections the fibre tracts were easily identified. The cells adjacent to the fibre tracts were morphologically nomal with an oblated hyperchromatic appearance. The cytoplasm of these cells was hypereosinophilic, consistent with the presence of coagulated proteins. Outside this small zone the cells showed less severe damage with unstained nuclei that retained their chromatin pattem and nucleoli. Occasionally areas of necrosis were found within the tumour. Features indicating apoptotic cell death were not found. In the patients with multiple tumours, of which only the largest tumour was lased, the findings in the other tumours were comparable to those in control material.

NADH staining revealed that NADH-diaphorase activity was high in normal liver, low in metastatic tumours and absent in the samples obtained from macroscopically coagulated tissue ( 7.3). Results with the NADH staining corresponded to the macroscopic observations: in samples from a 78 
macroscopically completely coagulated location no NADH-diaphorase was identifiable whereas in normal parenchyma or in tumour where laser treatment had failed NADH-diaphorase was always stainable. A sharp demarcation between oxidase positive and negative zone was visible in the sections taken from a transition zone between nomal tissue and macroscopical coagulated tissue. DNA fragments, stained with the TUNEL technique, were present in all sections, whether liver or metastasis, and coagulated macroscopically or not. Only in sections with macroscopic whitening of tissue and in areas which corresponded to the necrotic tissue in the H\&E sections, DNA fragments were found in more than $15 \%$ of the nucleated cells (Figure 7.3). In table 7.1 the completeness of coagulation is differentiated according to the sample locations together with conclusions on microscopical vitality.

Table 7.2 Macroscopic data of human colorectal metastases in the liver in which interstitial laser coagulation has been performed following and prior to surgical resection. Laser treatment was with $5 \mathrm{~W}$ per fibre during 6 minutes. Dimensions are given as $h$ and $w(\mathrm{~cm})$.

\begin{tabular}{|c|c|c|c|}
\hline Number of fibres & $\begin{array}{c}\text { Tumour } \\
\text { dimensions }\end{array}$ & $\begin{array}{l}\text { Coagulation } \\
\text { dimensions }\end{array}$ & $\begin{array}{c}\text { Position } \\
\text { coagulation relative to tumour }\end{array}$ \\
\hline \multicolumn{4}{|l|}{ Lased after resection } \\
\hline I & $2.9 \times 2.7$ & $3.2 \times 2.9$ & complete encircling \\
\hline 4 & $6.7 \times 5$ & $4.5 \times 3.8$ & within tumour \\
\hline 4 & $3.8 \times 3.1$ & $4.1 \times 3.6$ & complete encircling \\
\hline 4 & $4.9 \times 3.5$ & $5.5 \times 4.7$ & complete encircling \\
\hline 4 & $3.1 \times 2.8$ & $4.1 \times 3.3$ & complete encircling \\
\hline \multicolumn{4}{|l|}{ Lased prior to resection } \\
\hline 2 & $2.5 \times 2.6$ & $3.5 \times 2.5$ & complete encircling \\
\hline 4 & $3.5 \times 3.5$ & $4.4 \times 3.5$ & eccentric to tumour \\
\hline 4 & $3.0 \times 2.5$ & $4.9 \times 4.1$ & complete encircling \\
\hline 4 & $4.9 \times 3.3$ & $4.5 \times 3.8$ & within tumour \\
\hline 1 & $2.6 \times 2.1$ & $3.3 \times 3.0$ & complete encircling \\
\hline
\end{tabular}




\subsection{DISCUSSION}

In this study the vitality of liver metastases was investigated directly after ILC in human hepatic metastases. At microscopy we found no mitochondrial NADH activity nor DNA integrity in the areas that were coagulated at macroscopy. If a tumour was coagulated incompletely these two parameters correspondingly indicated vital tissue. The largest diameter of the volume of coagulation was $5.5 \mathrm{~cm}$, sufficient to completely coagulate the respective tumour of $4.9 \mathrm{~cm}$.

Morphological observations at microscopy do not allow unambiguous conclusions regarding the cellular vitality directly post laser treatment (Thomsen, 1991). A heat fixation occurs, leaving the tissue frame intact and initially also the morphology (van Hillegersberg et al, 1994). Nucleated cells have been described in H\&E stained biopsy specimens following ILC, that were found avital when these cells were studied with electron microscopy (Nolsoe et al, 1989). Using the activity of the mitochondrial enzyme NADH-diaphorase as marker for metabolism was first described by Gordon et al. (1996) in uterine leiyomyoma lased just prior to surgical excision. In agreement with our findings a good correlation between macroscopical coagulation and the area of absent mitochondrial metabolism was found.

In addition to metabolic activity the integrity of the DNA was used as a parameter for effect. The used technique stains DNA fragments form dissociated strands. This TUNEL technique has been developed to prove a specific patten of cell death, i.e. apoptosis, but has been shown to be a reliable early marker of necrosis (Gold et al, 1994). In a study, in which prostate was investigated 3-5 days after thermal coagulation with microwave irradiation, staining for the TUNEL fragments corresponded to apoptosis in the morphological specimen. In a single prostate examined 2 hours after thermotherapy the morphological signs of apoptosis were not visible whereas the TUNEL staining was positive for disintegrated DNA. This situation is comparable to the present study design in which also only the specific TUNEL staining pattern was found without the morphological signs of apoptosis.

Because of the study design a second factor with influence on cellular vitality has to be considered. All resection specimens, whether subjected to ILC prior or after resection, were manipulated surgically with flow occlusion during surgical resection to minimize blood loss from the parenchymal dissection plane. This flow occlusion may have added to the coagulation damage: ischemia causing cell death in areas critically but not completely damaged by coagulation. Disentangling of these factors is further complicated by our routine use of vascular inflow occlusion during $\mathrm{ILC}$ as desctribed in chapters 5 and 6 . This maneuver results in a 5 -fold increase in the 80 
volume of coagulation and is explained by the reduction in convection of heat and not merely by ischemia superposed on coagulation (Roggan and Muller, 1994).

Previously the effects of ILC prior to surgical resection have been investigated in breast, prostate and liver tumors (Mumtaz et al, 1996; Vogl et al, 1996; Boni et al, 1997). To allow the morphological features to develop, resection was performed 2-10 days after laser treatment. However, this is the first report where immunohistochemical prove is given for complete cell death in humans. The used ILC technique in the three previous studies also differed fundamentally from that of the present study. In all three studies ILC was performed with a single laser fiber and without vascular flow to the organ. This limited lesions size to maximal $3.5 \mathrm{~cm}$ compared to $5.5 \mathrm{in}$ our study. Nevertheless within these areas cell death as the result of coagulation was a universal finding.

Ex vivo tissue porcine liver is a frequently used model to assess the ILC effects with different laser parameters. As tumour and liver differ in colour, the optical characteristic might differ as well, and thus the thermal effect and resulting area of coagulation. Previously, van Hillegersberg et al. (1993b) found no net difference in light absorption coefficient between rat tumour and liver at 1064 $\mathrm{nm}$; scattering was only slightly higher in tumour. Consistently, the findings in chapter 4 in ex vivo porcine liver are comparable to results in this chapter in human tumour tissue in vivo with the same laser parameters. The ex vivo liver is thus an excellent model to study laser effects.

In none of the specimens a haemoraghic rim was found surrounding the whitish coagulation. This haemorraghic zone is a typical finding after laser coagulation in a variety of living tissues and is explained by thermal intravascular erythrolysis (Thomsen, 1995). In chapter 5, where the effect of ILC was evaluated directly after ILC in pigs this rim of 2-3 $\mathrm{mm}$ was always found whether or not the flow was occluded (Figure 5.3). This contrast may be explained by the lack of organ reperfusion after ILC in this study. This is evident in the tumours lased after resection but also applies to the tumours lased prior to surgical resection because of the vascular occlusion of the hepatic inflow during liver resection to minimise blood loss. Germer et al. (1997) have demonstrated with NADHdiaphorase staining that the lased hepatocytes in the haemoraghic zone are avital. Whether destruction of these cells is the result of reperfusion injury to cells already sublethally damaged by ILC remains to be investigated. In this situation the maximal volumes of coagulation may be slightly underestimated in the present study. 
Figure 7.3 Snap frozen sections stained for NADH-diaphorase activity: (A) metastatic tumour, (B) coagulated metastasis and (C) transition from coagulated liver parenchyma to vital liver parenchyma. The blue granules indicate NADH-diaphorase and thus mitochondrial activity (magnification 400x). (D) Paraffin embedded section stained for double stranded DNA breaks thereby identifying DNA fragmentation: Normal cells, absent, have green nucleoli whereas fragmented DNA appears brown. Microscopy of macroscopically coagulated tumour is shown (250X).
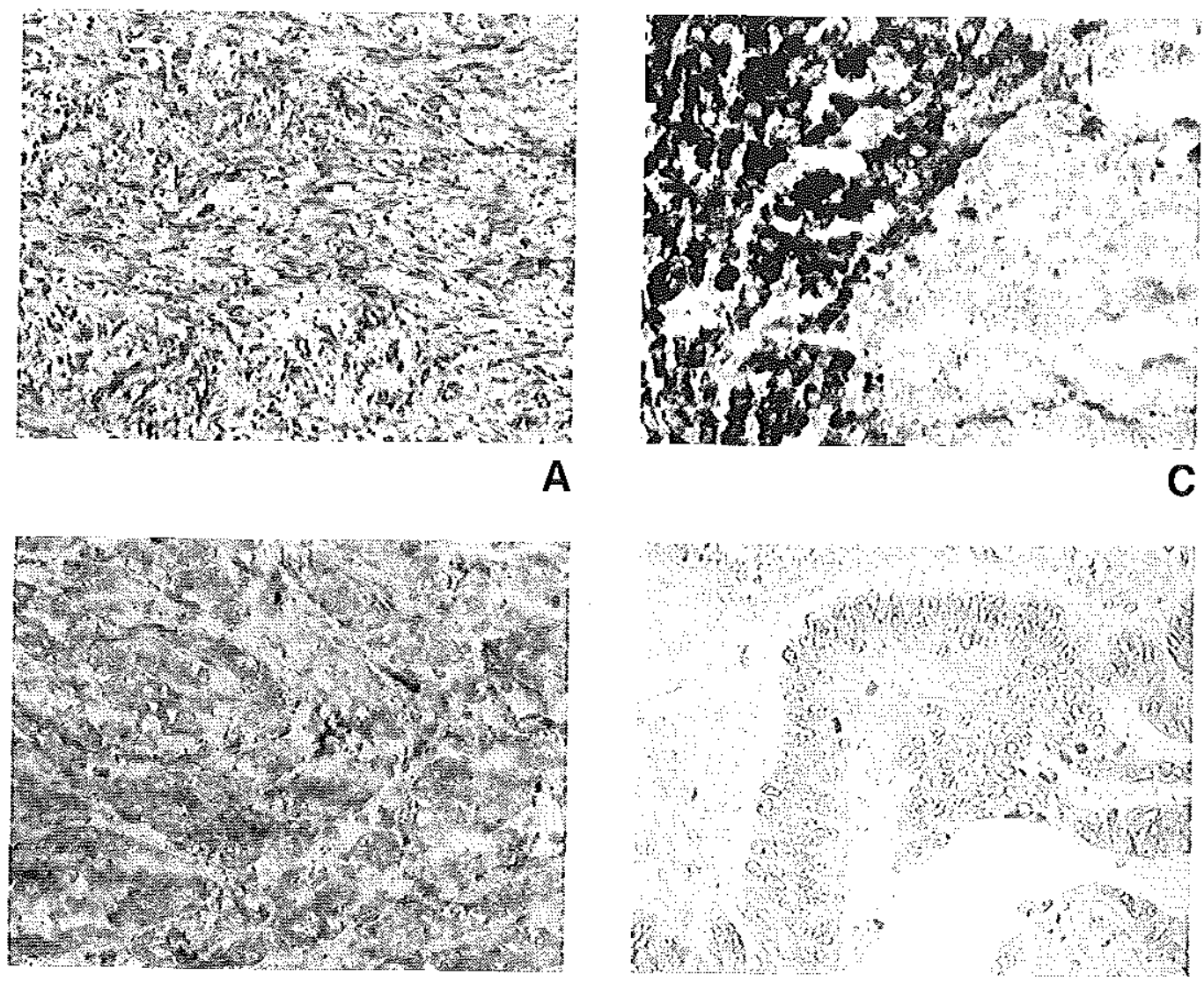

B

D 
In 3 of 10 tumours vital cells remained, in all instances outside the macroscopic area of coagulation. In two instances this was to be expected as the tumour diameter exceeded the limits of the current ILC technique. In one tumour the positioning of the four fibres was eccentric to the tumour resulting in an area of coagulation larger than the tumour but not completely encircling this tumour. This finding underscores the importance of the development of tools to monitor the introduction of the laser fibre(s) and the laser-induced effects during treatment.

In conclusion, it was found that 1) the volume of coagulation is comparable in normal liver and metastatic colorectal cancer and 2) no vital cells remain within the macroscopically coagulated whitish tumour after ILC using both a direct marker of cellular metabolism as well as an indicator of DNA integrity. 



\section{(0) Critical temperature and heating time for coagulation damage: Implications for Interstitial laser coagulation}

J Heisterkamp, R van Hillegersberg \& JNM IJzermans. Lasers in Surgery and Medicine, 1999: 25(3);257-262.

\subsection{INTRODUCTION}

Interstitial laser coagulation (ILC) is an emerging treatment modalify for patients with solid lesions in parenchymal organs such as hepatic and brain tumours. Upon absorption of the laser light (either Nd:YAG or Diode) several thermal effects may take place. Coagulation is defined as the irreversible thermal damage of tissue proteins at temperatures between $55^{\circ} \mathrm{C}$ and $95^{\circ} \mathrm{C}$. If the temperature exceeds $100^{\circ} \mathrm{C}$, water vaporisation followed by tissue carbonisation and ablation occurs (Table 1.1). Protein denaturation occurs of 2 stages: unfolding and subsequently aggregation of the protein of which the latter is irreversible (de Wit and Swinkels, 1980; Verheul, 1998). From studies on dissolved individual proteins it is known that there is a large variability between different proteins in the temperature at which coagulation occurs $\left(\mathrm{T}_{\mathrm{c}}\right)$ in a range between $48^{\circ} \mathrm{C}$ and $90^{\circ} \mathrm{C}$ (Joly, 1965; Ritchie et al, 1994). Cell viability is related to the thermostability of essential proteins with a $\mathrm{T}_{\mathrm{c}}$ around $60^{\circ} \mathrm{C}$ (Lepock et al, 1990; Lepock et al, 1993; Xu and Qian, 1995). Formation and extent of coagulation are dependent on the period of elevated temperature in this range. The crux of ILC is that the magnitude of protein denaturation is sufficient to result in cell death. Although it is generally assumed that in solid tissue and tumour, heated at $60^{\circ} \mathrm{C}$ for several minutes, complete protein denaturation occurs, there are no supporting experimental data for ILC of tumours.

So far, exact knowledge of the critical temperature has not been relevant as tissue temperature could not be measured precisely enough in real-time. Recent advances in magnetic resonance temperature-assessment (MR-T) will warrant more precise temperature estimates in an unlimited number of locations and thus allow thermometry to monitor and to control ILC (Jolesz et al, 1998). 
This development actualises the question of the optimal combination of temperature and exposure time necessary for tissue coagulation in solid tumour.

\subsection{MATERIALS AND METHODS}

Solid tumour samples were used. The effects of combinations of 4 temperatures $\left(40^{\circ} \mathrm{C}, 50^{\circ} \mathrm{C}, 60^{\circ} \mathrm{C}\right.$ or $80^{\circ} \mathrm{C}$ ) and 4 different exposure times $(3,6,12$ or $24 \mathrm{~min}$ ) were investigated (16 groups). Combinations were replicated in five fold. Negative control samples were stored at $4^{\circ} \mathrm{C}$ and positive controls consisted of microwave treated samples (cooking for 1 minute). Cell viability of the tumor sample after heating was assessed using 3 methods: 1) Trypan blue exclusion assay in collagenase/dispase dissociated samples (Neumann et al, 1991), 2) mitochondrial NADH activity in snap frozen samples (Eriksson et al, 1984) and 3) outgrowth for 2 weeks under the renal capsule in Wag/Rij rats (syngeneic to the used tumour) (Venesmaa and Ylikorkala, 1991).

Ether anaesthesia was used for all surgical procedures. The experimental protocol adhered to rules laid down by the Dutch Animal Experimentation Act and was approved by the Committee on Animal Rescarch of the Erasmus University Rotterdam.

\section{Tumour line and sample harvest}

CC531 is a moderately differentiated, weakly immunogenic colonic adenocarcinoma induced in a WAG/Rij rat by administration of 1,2-dimethylhydrazine (Marquet et al, 1984). A cell line has been established from this carcinoma which is maintained by serial passage after trypsinisation in culture medium. CC531 tumour cells were cultured in RPMI 1640 medium supplemented with 5\% foetal calf serum, glutamin $(2 \mathrm{mM})$ and penicillin $\left(10^{5} \mathrm{U} / \mathrm{L}\right)$, and passaged twice weekly [all supplements Gibco, Paisley, UKJ. Before use, cells were trypsinised, centrifuged and resuspended in medium. To grow solid tumours, $1 \times 10^{8} \mathrm{CC}-531$ tumour cells were injected in the right and in the left flanks of inbred WAG/Rij rats [Harlan-CPB, Zeist, The Netherlands]. After 6 weeks tumour volume in both flanks had reached a volume of approximately $2.5 \mathrm{~cm}^{3}$ and the mass was isolated aseptically with a scalpel from the outer membrane. Subsequently the tumour was divided into samples of approximately $8 \mathrm{~mm}^{3}$ and immersed in medium on ice till further procedures. The necrotic centre of the tumour was not used for tumour sample harvest. 


\section{Heating}

The obtained tumour samples were immersed in quadruplicate in tubes [Eppendorf, Hamburg, Germany] and filled up with $1 \mathrm{ml}$ medium at $4^{\circ} \mathrm{C}$. Immediately the tubes were placed in a thermostat controlled water bath [Dépex, De Bilt, The Netherlands]. Thirty seconds sufficed to raise the core of the samples to the temperature of the water bath (as determined in pliot studies with four fiberoptic thermosensors [SMM interstitial probe and model 790, Fluoroptic Thermometry System, Luxtron, Santa Clara (CA), USA] positioned I) in the centre of sample 2) attached to the sample 3) at the tube's wall and 4) at the transition medium to air respectively). After these 30 seconds the randomised exposure time was counted. Immediately following heating, the tubes were stored on ice till further processing.

\section{Evaluation}

Vitality of three of the four samples in each tube was evaluated, each with a different method.

\section{Trypan blue exchision}

One tumour sample was dissociated by sonication in a water bath at $37^{\circ} \mathrm{C}$ in medium containing 1.5 $\mathrm{mg} / \mathrm{ml}$ Collagenase/Dispase [Sigma, Steinheim, Germany]. After incubation in Trypan Blue solution the percentage of dead cells was assessed and expressed as those incorporating the blue pigment (De Luca, 1965).

\section{NADH histochemistry}

Staining with nitro blue tetrazolium (NBT) can be used to stain NADH-diaphorase activity which is a measure for mitochondrial metabolism. Upon cell death the activity of NADH-diaphorase subsides (Neumann et al, 1991). Snap-frozen samples were stored at $-80^{\circ} \mathrm{C}$ and serial sections of 8 $\mu \mathrm{m}$ were cut a cryostat. Sections were picked up onto clean glass slides. Incubation solution consisted of $9 \mathrm{mg} \mathrm{NADH}, 3 \mathrm{ml}$ phosphate-buffered-saline, $12.1 \mathrm{ml}$ distilled water and $35 \mathrm{mg}$ NBT [Sigma]. Slides were covered with $100 \mu$ solution and incubated for 60 minutes at $37^{\circ} \mathrm{C}$ at aerobic conditions. Slides were washed with distilled water, mounted in a formaline/macrodex solution medium and covered with a glass cover slip. With light microscopy mitochondrial activity was visible as blue staining. Proportion of staining was scored: $1,<25 \% ; 2,25-49 \% ; 3,50-74 \%$ or 4 , $>75 \%$ ). 


\section{Renal subcapsular assay}

Individual tumour samples were weighted. After a small midline laparotomy, both poles of the left and right kidney were exposed. The capsule at each pole was dissected to allow the placement of one tumour sample at each pole. The laparotomy was closed in one layer. Two weeks after implantation, all animals were sacrificed. Growth of the tumour sample was determined as the wcight difference between the enucleated lump and the implanted sample.

\section{Statistics}

All numerical data are expressed as mean \pm standard error of the mean (SEM), and tested for difference between the experimental groups with Student's $t$ test. Differences between groups associated with exposure duration or temperature of heating were analysed using a two-way of analysis of variance test with both variables as factor (Venables and Ripley, 1994). Results were considered statistically significant at $P<0.05$.

\subsection{RESULTS}

All animals tolerated the experimental procedures. Tumour take varied between the five donor rats and left and right thighs but the net yield sufficed for the experiments.

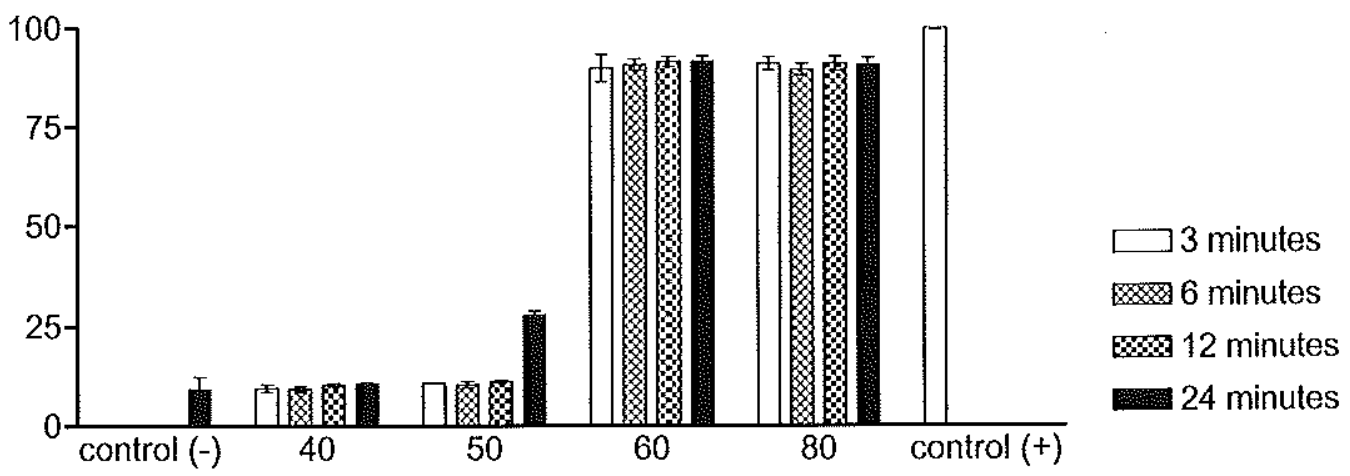

Temperature

Figure 8.1 Average percentage of cells incorporating Trypan blue solution. Bars indicate standard error of the mean and results are the mean of 5 experiments. 


\section{Trypan blue exclusion}

In general, the yield of dissociated cells was low, especially in the samples heated at $80^{\circ} \mathrm{C}$ for all exposures durations and positive controls. Per view a minimum of $1.3 \times 10^{6}$ cells was always visible. In Figure 8.1 the results are shown. Heating at $40^{\circ} \mathrm{C}$ at all exposure durations and at $50^{\circ} \mathrm{C}$ except for the longest duration $(24 \mathrm{~min})$ did not affect viability compared to the negative controls. The number of viable cells after heating at $50^{\circ} \mathrm{C}$ for 24 minutes dropped to $73 \%$. At $60^{\circ} \mathrm{C}$ and $80^{\circ} \mathrm{C}$, cell viability was decreased to $12 \%$. No statistically significant differences were found between heating at $60^{\circ} \mathrm{C}$ and $80^{\circ} \mathrm{C}$, irrespective of exposure duration $(P=0.78)$.

\section{NADH histochemistry}

In samples heated at $40^{\circ} \mathrm{C}$ for all exposure durations and at 3,6 and 12 minutes heating at $50^{\circ} \mathrm{C}$, the number of cells exposing mitochondrial NADH activity was always $>75 \%$. In samples heated at $60^{\circ} \mathrm{C}$ and $80^{\circ} \mathrm{C}$, barely any blue staining was detectable, indicating the absence of mitochondrial metabolism (Figure 8.2).

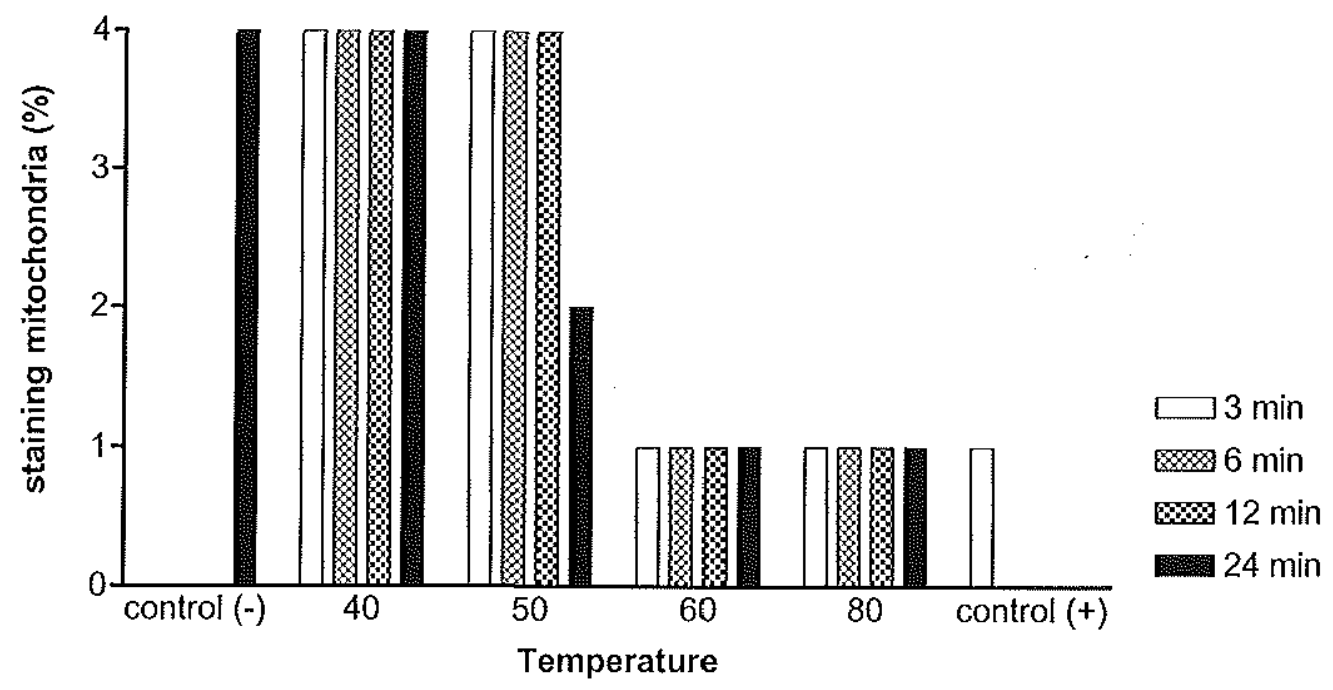

Figure 8.2 Proportion of cells staining positive for mitochondrial NADH activity, scored in 4 groups: 1 , $<25 \% ; 2,25-49 \% ; 3,50-74 \%$ or $4,>75 \%$ ). Results are the mean of 5 experiments. 


\section{Renal subcapsular assay}

Median (range) sample weight prior to implantation was $51.8 \mathrm{~g}(41.3-60.9)$ with an even distribution between the different groups (all $F>0.56$ ). Samples heated at $40^{\circ} \mathrm{C}$ and $50^{\circ} \mathrm{C}$ showed the typical aspect of proliferating CC531 tumour (Marquet et al, 1984). These samples had a proliferate growth and had increased in weight. Samples heated at $60^{\circ} \mathrm{C}$ and $80^{\circ} \mathrm{C}$ had a cystic appearance and did not show growth in any of the experiments (Figure 8.3).

\subsection{DISCUSSION}

In this study the critical temperature and exposure duration for tissue coagulation and tumour death were investigated for small tumour samples. It was found that a temperature above $50^{\circ} \mathrm{C}$ and below $60^{\circ} \mathrm{C}$, applied for a short duration ( $<3 \mathrm{~min}$ ) results in sufficient coagulation for complete cell death. These data are in line with studies with protein suspensions and cell solutions (Lepock et al, 1990; Lepock et al, 1993; Xu and Qian, 1995). As the accuracy of non-invasive temperature measurements during ILC for tumours has increased in recent years, the present results may be used to determine the degree of coagulation at a certain point in the heated tissue.

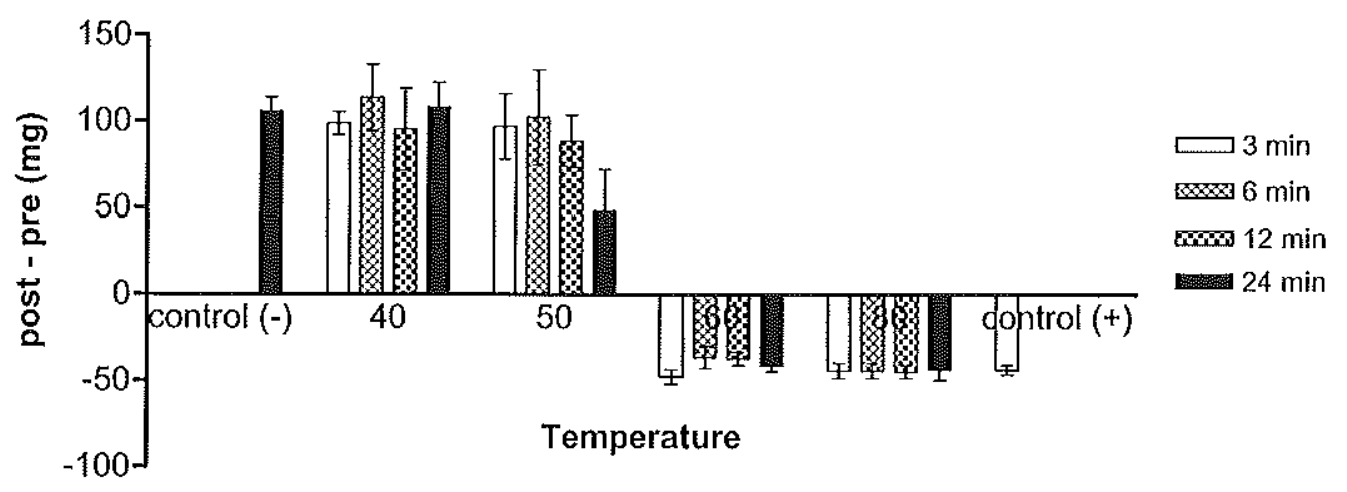

Figure 8.3 Mean weight change after 2 weeks of implantation under the renal capsule (weight enucleated lump minus weight of implanted sample). Bars indicate standard error of the mean and results are the mean of 5 experiments. 
Three cstablished methods of assessing cellular viability and anti-tumour effects were used (Eriksson et al, 1984; Neumann et al, 1991; Venesmaa and Ylikorkala, 1991). Direct cell death was examined using NADH staining in snap frozen samples. This technique has been shown to be superior for this particular purpose, compared to traditional assessment of morphological changes with light microscopy (Eriksson $e t a l, 1984$ ), and has been used before to investigate laser induced histological changes (Neumann et al, 1991). Membrane integrity was tested using Trypan blue exclusion in dissociated tissue samples. The crux of ILC, namely complete cell death, was studied with outgrowth at long term of a heated sample, implanted in a healty rat (Venesmaa and Ylikorkala, 1991). The results of the three methods were concordant.

In the present study tissue samples were heated in a water bath to obtain homogeneous heating. Castrèn-Person et al. (1996) noted in heat sensitivity studies of cell cultures a difference in the effect between the laser-induced hyperthermia temperatures and water bath heating at the same temperature in the control group They concluded that apart from heat induced cytotoxicity also a direct light toxicity may play a role. In ILC, however, temperatures remote from the laser tip are determined by heat conduction (Svaasand et al, 1990). Therefore, we take the view that the present study represents the situation at locations of interest in the tumour: at the border and near important structures within the organ. In agreement with the present study Castrèn-Person et al. found that the largest proportion of cell killing is executed between $49^{\circ} \mathrm{C}$ and $59^{\circ} \mathrm{C} \mathrm{(1996).}$

For traditional hyperthermia, aiming at temperatures between $42^{\circ} \mathrm{C}$ and $45^{\circ} \mathrm{C}$, cellular viability of heated tissue has been investigated previously. Ikeda et al. studied histological changes in canine brain after heating for prolonged periods up to 60 minutes (1994). Heating at a temperature of $44^{\circ} \mathrm{C}$ during more than 45 minutes resulted in necrosis, whereas at $42^{\circ} \mathrm{C}$ for 30 minutes no changes were found in tissue. The results of the latter combination of time and temperature are concordant to the results in the present study.

In a different set-up Schulze et al. (1998) came to a very precise determination of the threshold for tissue coagulation. Using magnetic resonance imaging to monitor interstitial temperatures, the isotherm ranging between $60^{\circ} \mathrm{C}$ and $65^{\circ} \mathrm{C}$ was found to correspond to the largest dimensions of the coagulated tissue. However, as golden standard, the investigators used a MRI technique of which the accuracy has not yet been established (Jolesz et al, 1998). Therefore this threshold should be interpreted with caution.

In the present study the temperature intervals were large and further studies are required to obtain a 
more precise determination of the exact temperature and exposure duration at which cell death occurs. In this respect, Mordon et al. (1995) reported on an interesting tool. Liposomes were filled with fluorescent dyes and injected intravenously in rats prior to ILC in liver. These liposomes were pharmaceutically engineered to release the dyes with temperature increase. The amount of drug release was found to be lineary to the duration of exposure. Liposomes can be tailored to release their contents at any given temperature and might thus be useful to study the exact temperature at which tissue coagulation occurs. As a guide the present results can be used. 


\section{0 \\ Accuracy of MR phase mapping for temperature monitoring during ILC in the liver at rest and with simulated respiration and in vivo}

Adapted from: J Heisterkamp, NAA Matheijssen, R van Hillegersberg, JJ van Vaals, JS Laméris, J Stoker \& JNM IJzermans. Accuracy of MR phase mapping for temperature monitoring during ILC in the liver at rest and with simulated respiration Magnetic Resonance in Medicine, 1999, 41; 919-925

\subsection{INTRODUCTION}

The success rate of interstitial laser coagulation (ILC) in coagulating entire tumours depends on two factors, 1) the volume of coagulation has to be larger than the tumour volume and 2) the process of coagulation has to be monitored to control destruction of the entire tumour without damaging adjacent normal structures such as hepatic veins and hilar structures.

Early enthusiasm for ultrasound and computed tomography in imaging ILC (Steger et al, 1992b; Amin et al, 1993c; van Hillegersberg et al, 1993a) faded with the advent of powerful and rapid MRI techniques. T1-weighted imaging was reported to be very useful in monitoring laser treatment in patients with hepatic metastases (Roberts et al, 1994; Vogl et al, 1995; Vogl et al, 1997). Disappointing results of carefully performed ex vivo and in vivo animal experiments (Castro et al, 1990; Tracz et al, 1992; Pushek et al, 1995; Fried et al, 1996; Schwabe et al, 1997), however, prompted investigations to other MR sequences.

The chemical shift or proton-resonance frequency, also referred to as phase-shift mapping can be used to measure temperature changes; in 1995 this concept was claborated upon, showing the feasibility and accuracy of this subtraction technique (De Poorter et al, 1994; Ishihara et al, 1995). Nevertheless, as a subtraction technique, scans at exactly the same location are required, making the technique prone to respiration-induced artefacts.

The purpose of the present study was to investigate the accuracy of the MR phase-shift mapping 
technique at rest and in motion, by simulating respiration motion. In experiments studying the MR performance with a homogenous medium, heated gel was used. An ex vivo porcine liver model was used to study phase-shift mapping during laser application. In continuation, pilot studies were performed in pigs.

\subsection{MATERIALS AND METHODS}

MR protocol and image analysis

Phase-shift mapping was performed on a commercially available 1.5 Tesla MR scanner [Gyroscan ACS-NT, Philips Medical Systems, Best, The Netherlands]. A turbo field ccho sequence was used (TR $8.1 \mathrm{msec}$, TE $3.4 \mathrm{msec}$, FA $60^{\circ}$ and $75 \%$ RFOV of $250 \mathrm{~mm}$ with a $128 \times 128$ matrix; $800 \mathrm{msec}$ per slice acquisition). In a pilot study the sequence was optimised to a homogeneous and reproducible phase image in dynamic series of liver at rest, aiming at a scanning time $<1$ sec per slice.

A reference scan was performed for several slice directions to acquire a baseline image (both modulus and phase). The temperature changes in the model were measured using the same sequence and slices in a dynamic scan after anti-aliasing and noise filtering. Phase images were compared to baseline and the phase differences (shifts) were calculated using a tempcrature coefficient of 0.88 degree phase shift per ${ }^{\circ} \mathrm{C}$ as described previously (Stollberger et al, 1998) [special temperature mapping software, Philips Medical Systems]. With this software, temperature images were displayed in real-time as overlays to the orthogonal baseline modulus image, using three colours corresponding with 3 adjustable temperature ranges (Figure 9.3). Additionally, these temperature images were exported to a personal computer and analysed using the SCIL-image package [TNO Institute for Applied Physics, Delft, The Netherlands].

\section{Respiration simulation}

All experiments were performed with the object placed on a custom-built table which could be moved along the axis of the magnet for the in motion studies. The mechanical motion was airpressure driven with controllable frequency and duration of the stops to simulate inspiration and expiration. The moments of maximum excursion were directed electronically to the ECG sensor of the MR-system and used to trigger scanning. In these experiments respiration was simulated as 12 excursions per minute with $40 \mathrm{~mm}$ relocation and $1 \mathrm{sec}$ stops at both ends. The pattern of stops was not abrupt but smooth as in a sinusoidal pattern. Scanning was triggered only when the table was in 
the feet position, simulating maximal inspiration. Images were made at the same position with respect to both the object frame as well as the magnet frame.

\section{Gel studies}

Four large volume syringes were filled with $25 \mathrm{ml}$ ultrasound gel [2 RTC, $\mathrm{E} * \mathrm{Z} * \mathrm{EM}$, Dordrecht, the Netherlands] mixed with $5 \mathrm{ml}$ of dissolved (20\%) Gadodiamide [Ommiscan, Nycomed Imaging, Oslo, Sweden] and centrifuged to release air bubbles. Three syringes were heated in a microwave oven to achieve starting temperatures of approximately $40^{\circ} \mathrm{C}, 50^{\circ} \mathrm{C}$ and $60^{\circ} \mathrm{C}$. The fourth syringe remained at room temperature. Syringes were placed in a polystyrene foam block and placed in the MR with the 4 tubes perpendicular to the axis of the magnet. In each tube an optical temperature measurement fibre was placed [SMM interstitial probe and Model 790, Fluoroptic Thermometry System, Luxtron, Santa Clara (CA), USA] (Taber and Hayman, 1992). Probe tips were blanched to exclude absorption of the laserlight and recalibrated according to the manufacturers instructions.

The cooling of the gel in the syringes was measured using MR temperature mapping, acquiring an MR phase image every minute during 30 minutes. All syringes were imaged with 3 orthogonal slices ( 6 slices in total). Each experiment was replicated 5 fold at rest and 5 fold in motion. For all temperature images and each plane scanned the average temperature and standard deviation (SD) were calculated

Figure 9.1 Survey scan of the transversal slice through the liver at the position of the laser fibre (L) and 3 parallel thermofibres (T1-T3). Both laser fibre and thermoprobes are guided through small bore iv cannulas with magnetic markers (arrow). After confirming correct positioning these cannulas were redrawn and a second survey scan was made and used as baseline for calculation phase-shift differences in the dynamic series. Bar indicates $1 \mathrm{~cm}$.

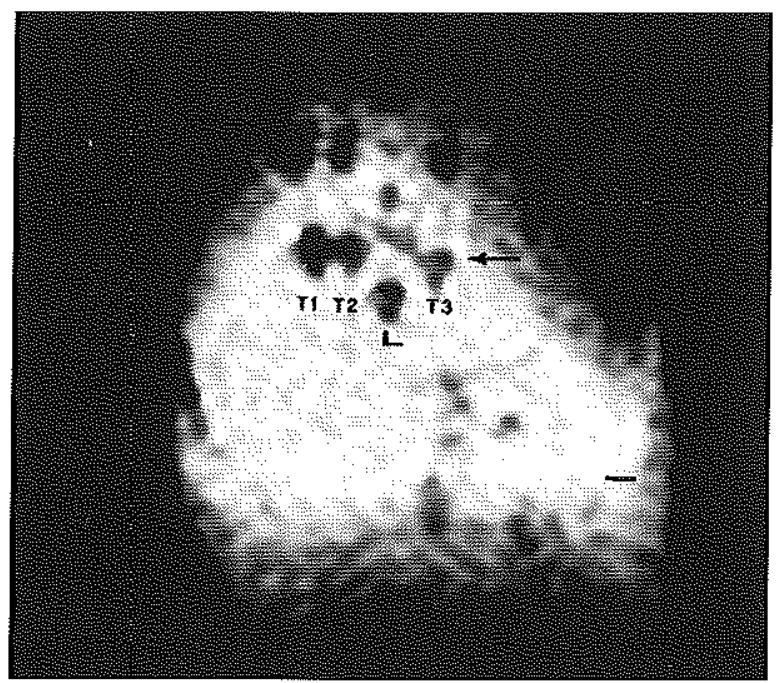




\section{Liver, ex yivo, studies}

Five small borc cannulas [14G, Adsyte, Becton Dickinson, Madrid, Spain] were marked at the distal end and at $2 \mathrm{~cm}$ proximal from the distal tip with small amounts of $\mathrm{Fe}_{2} \mathrm{O}_{3}$ doped material functioning as MR landmarks $\left(1 \mathrm{~mm}^{2}\right)$. Lobes of porcine livers were heated to body temperature (mean $34.5^{\circ} \mathrm{C}$ range: $31.5^{\circ} \mathrm{C}-38^{\circ} \mathrm{C}$ ) using two warm-water mattresses. A single lobe was positioned at the table with the longest axis along the magnets direction ( $\mathrm{z}$ axis). The first cannula was introduced centrally into the liver at an angle of approximately $20^{\circ}$, through which a laser fibre with a cylindrical diffusing tip of two $\mathrm{cm}$ length [Lightstic ${ }^{\mathrm{TM}}$, Cardiofocus, West Yarmouth, Ma, USA] was guided. The laser fibre was marked to ensure positioning of the diffusing tip precisely between the MR markers. Three cannulas were inserted parallel to the laser fibre at $1 \mathrm{~cm}$ to the left and 1 and $2 \mathrm{~cm}$ to the right. Another cannula was positioned remotely from the laser fibre. Through these 4 extra cannulas, optical temperature measurement fibres were introduced [Luxtron]. The correct position of the thermofibres half way the diffusing tip was confirmed with a survey scan and relative positions were documented (Figure 9.1). In addition 3 orthogonal planes through the laser fibre and through the 3 parallel thermofibres were established and confirmed. After withdrawing the cannulas, leaving the laser fibre and thermoprobes in place, a second survey scan was acquired as reference scan for phase-shift calculations.

Twenty-five dynamic scans were acquired of the 6 slices at intervals of 30 seconds. The laser [ $\lambda=1064 \mathrm{~nm}$; model KTP/YAG 830, Laserscope, San José (CA) USA] was turned on after 2 minutes for the next 6 minutes at a power of 5 Watt (2160 Joules) to induce coagulation damage. As shown in chapter 3 , reproducible results were found with this fibre and laser parameters.

Following laser application cooling was monitored for 4 minutes. Immediately following the dynamic series, the liver was removed from the bore and sliced along and perpendicular to the laser fibre. Coagulation was determined as the whitish colour change compared to normal coloured parenchyma. Length (along fibre) and largest diameter (orthogonal to fibre) of the area of coagulation were measured using callipers.

The temperature images were analysed as follows: in all scans the positioning of the 4 thermoprobes was assessed using the initial survey scan (Figure 9.1). In the temperature images of all scanned slices, average temperature and SD were calculated of an area of $3 \times 3$ pixels surrounding the location of the 4 thermoprobes. In the transversal and coronal slices of the first scan following 
laser application, the areas with temperature above respectively $40^{\circ} \mathrm{C}, 50^{\circ} \mathrm{C}, 60^{\circ} \mathrm{C}, 70^{\circ} \mathrm{C}$ or $80^{\circ} \mathrm{C}$ were isolated. The longest axis and shortest axis of the fitted ellipsoid were calculated.

\section{In vivo studies}

In three female pigs of pure Yorkshire breed $(30-40 \mathrm{~kg})$ the MRI scamning protocol was evaluated. Anaesthesia was induced with azaperon (im, $0.05 \mathrm{ml} / \mathrm{kg}$ ) and after administering ketamine (im, 10 $\mathrm{mg} / \mathrm{kg}$ ) and thiopental (iv, $10 \mathrm{mg} / \mathrm{kg}$ ) the animals were intubated and ventilated artificially with $100 \%$ oxygen. During ventilation, anaesthesia was maintained with pancuronium (iv, $4 \mathrm{mg}$ ).and fentanyl (iv, $0.1 \mathrm{mg}$ ). After the experimental procedures the pigs were killed without recovery from the anaesthesia with a bolus injection of pentobarbital (iv). The experimental protocol adhered to rules laid down in the Dutch Animal Experimentation Act and was approved by the Committee on Animal Research of the Erasmus University.

At the operating theatre a subcostal incision was made to dissect the hepatoduodenal ligament. The portal vein was controlled with a vesselloop and the abdomen temporarily closed for transportation to the MR suite.

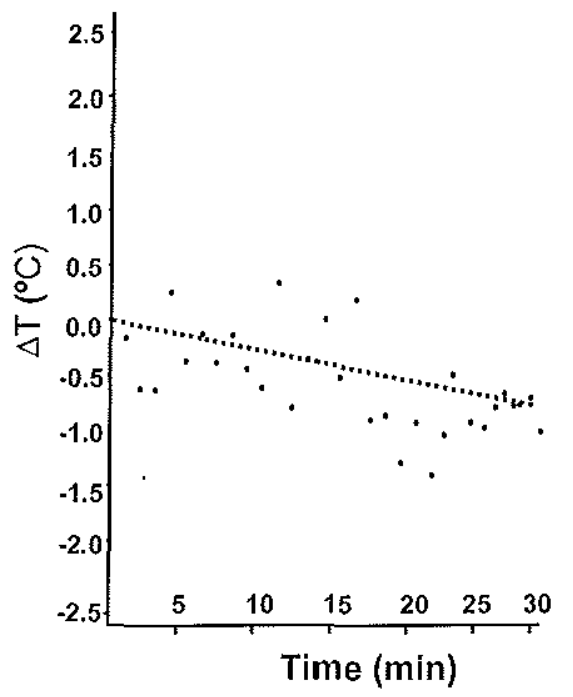

Figure 9.2 Typical reproduction of the difference between MR temperature and thermoprobe assessed temperature $(\Delta T)$ over time in a gel filled syringe scanned in the transversal plane. The dotted line represents the fitted regression equation and single points are calculated differences. Starting temperature in this particular case was $42.5^{\circ} \mathrm{C}$. 
The animal was positioned head first in the MR bore. A cylindrical diffusing fibre [Cardiofocus] encased in a transparent catheter [Powerapplicator, Somatex, Berlin, Germany] was inserted in the left or right central liver lobe. The optical temperature measurement fibres were positioned through cannulas as in the ex vivo studies: $1 \mathrm{~cm}$ to the left and 1 and $2 \mathrm{~cm}$ to the right from the laser fibre and a fourth fibre remote from the laser fibre. With the vesselloop the portal vein was snared and a survey scan was made to confirm the correct positions of the fibre. The identical 6 scanning planes were used as in the ex vivo study. After withdrawing the cannulas, leaving the laser fibre and thermoprobes in place, a second survey scan was acquired as reference scan for temperature induced phase-shift calculations.

Forty scans were acquired of the 6 slices at intervals of 30 seconds. The laser [Laserscope] was turned on after 2 minutes for the next 12 minutes at a power of 20 Watt (2160 Joules) to induce coagulation damage. During laser treatment the catheter was irrigated with $5 \% \mathrm{NaCl}$ at room temperature (Vogl et al, 1998). Pilot studies in ex vivo liver tissue with a similar design as chapter 3 showed reliable results with these fibre, catheter and laser parameters (not reported).

Following laser application, cooling was monitored for 8 mimutes. In each animal two laser applications were performed. After euthanasia, the liver was excised and the dimensions of the coagulation damage was determined as the whitish colour change compared to normal coloured parenchyma. Length (along fibre) and largest diameter (orthogonal to fibre) of the area of coagulation were measured using callipers. Dimensions were compared to the obtained MRthermography registration.

\section{Data handling and statistical analysis}

The MR and thermofibre measurements of each dynamic scan in the ex vivo studies were calculated relative to the initial temperature of each respective series. Also corrected MR values were calculated by subtracting the phase shifts at the reference location: being the syringe at room temperature in gel study and the remote thermoprobe in the liver study. Subsequently, the difference between MR-values and themoprobe values $(\Delta T)$ and between corrected MR-values and thermoprobe values $\left(\Delta \mathrm{T}_{\text {corrected }}\right)$ were calculated within each slice of each dynamic scan.

$\Delta \mathrm{T}$ and $\Delta \mathrm{T}_{\text {corretid }}$ were tested for deviation from 0 during the dynamic series, hypothesising that $\mathrm{MR}$ phase-mapping was comparable to thermofibre measurements $\left(\mathrm{H}_{0}\right)$. For each replication $(n=40$ for gel studies and $n=40$ for liver studies: at rest and in motion, with 4 temperature ranges per location 
of thermofibres and 5 replications) a regression model in time (30 time points in gel and 24 in liver per dynamic series) was fitted for differences with the $\Delta \mathrm{T}$ or $\Delta \mathrm{T}_{\text {corrected }}$ as variable. Because the $\mathrm{MR}$ software is calibrated by the thermoprobe value in the reference scan $(\Delta \mathrm{T}=0$ at $t=0)$ no intercept $(\alpha)$ was calculated, leaving the slope $(\beta)$ and the error of this estimate in the regression equation. The reciprocal of squared standard deviations $\left(1 / \mathrm{SD}^{2}\right)$ of averaged $\mathrm{MR}$ temperatures of the entire sectioned planes (gel) or of the $3 \times 3$ pixels surrounding the thermoprobes (liver) served as internal weight of the respective regression analyses.

The so obtained standardised slope values for the gel study were tested for co-variance with at rest or in motion as factor and initial temperature as co-variable, whereas the slope values of the liver series were analysed in a two-way analysis of variance (ANOVA) with both at rest or in motion and the location of thermofibre as factor. To test for differences between the fitted regression slopes, a variance ratio test (F-test) was performed (Venables and Ripley, 1994).

To assess correspondence between dimensions of coagulation with corresponding MR isotherms, analysis of correlation for non-parametric data was used (Spearmans' rank-order correlation test for a $95 \%$ confidence interval). To test for differences between series at rest and in motion in parametric data (initial temperature of gel and liver and dimensions of the induced areas of coagulation) Student's $t$ test was used.

Accuracy of the technique is expressed as $\Delta \mathrm{T}$ and $\Delta \mathrm{T}_{\text {corrected in mean }}{ }^{\circ} \mathrm{C}$ per minute $\left({ }^{\circ} \mathrm{C}\right.$ min $\left.{ }^{-1}\right)$. Continuous data are given as median (min.max) or mean (SD) as indicated and differences with a $P$ value $\leq 0.05$ were considered statistically significant. The S-Plus software package was used for all statistical computations [version 3.3 for MS-Windows, Mathsoft Inc., Seattle (WA), USA]

\subsection{RESULTS}

\section{Studies in gel}

The initial temperatures of 3 heated tubes and of the syringe at ambient temperature were not significantly different between series performed at rest or in motion $(P=0.11)$. Results were similar for all slice directions, therefore only data from the transversal plane are presented.

In all dynamic series the MR calculated temperature drifted away from thermoprobe measurements (a typical example is shown in figure 9.2). For at rest the mean (SD) $\Delta \mathrm{T}$ was $0.06^{\circ} \mathrm{C} \min ^{-1}(0.12)$ vs. $0.31{ }^{\circ} \mathrm{C} \min ^{-1}(0.32)$ in motion $(P=0.002)$. Also, the mean $\Delta \mathrm{T}_{\text {corrected }}$ (using corrected $\mathrm{MR}$ 
values) was significantly smatler at rest $[-0.15(0.28)$ vs. $-0.28(0.28), P<0.002]$. Differences between $\Delta \mathrm{T}$ and $\Delta \mathrm{T}_{\text {corrected }}$ were not significant within the at rest or in motion condition (respectively $P=0.96$ and $P=0.24$ ), see Table 9.1 and Figure 9.4).

True residual standard error of average temperature (dispersion around the fitted drift, i.e. systematic error) was significantly smaller at rest versus in motion $(P<0.001)$ but did not differ significantly between the respective temperature ranges $(P=0.82)$. The standard deviation of the averaged temperature within the scanned sectional planes was not different between at rest or in motion $\left(1.4^{\circ} \mathrm{C}\right.$ vs. $\left.1.2^{\circ} \mathrm{C}, P=0.46\right)$. No relation in time was found for these values $(P=0.45)$.

\section{Liver, ex vivo, studies}

No significant differences in start liver temperature were found between experiments at rest versus in motion $(P=0.25)$ and between central and remote locations in the individual experiments $(P=$ 0.90 ). Average length of the coagulated lesions (produced with $5 \mathrm{~W}$ during 6 minutes) at rest was $2.4 \mathrm{~cm}(0.3)$ and $2.2 \mathrm{~cm}(0.2)$ in motion $(P=0.21)$. Also differences in diameter of the lesions were not significant $(1.7 \mathrm{~cm}(0.2)$ at rest vs. $1.8(0.2)$ in motion $(P=0.49)$.

Results were similar for all slice directions, therefore only data from the transversal plane are shown (Figure 9.4, Table 9.1). Accuracy was not significantly different between at rest and in motion: mean $\Delta \mathrm{T}$ was $0.19^{\circ} \mathrm{C} \min ^{-1}(0.29)$ and $0.14^{\circ} \mathrm{C} \min ^{-1}(0.35)$ respectively, $P=0.31$. Deviation per minute when the MR output was corrected for phase-shifts in the constant did not differ significantly between the two experimental conditions (mean $\Delta \mathrm{T}_{\text {corrected }}$ was $-0.04{ }^{\circ} \mathrm{C} \mathrm{min}^{-1}$ $(0.34)$ at rest vs. $-0.0 \mathrm{I}^{\circ} \mathrm{C} \mathrm{min}{ }^{-1}(0.34)$ in motion, $\left.P=0.41\right)$. Differences between $\Delta \mathrm{T}$ and $\Delta \mathrm{T}_{\mathrm{c}}$ were not significant within the at rest or in motion experiments (respectively $P=0.39$ and $P=0.74$ ). Results were neither influenced by the location of the thermoprobe $(P=0.068)$ nor by initial temperature of the liver $(P=0.47)$. Standard deviations of the averaged temperature of the $3 \times 3$ pixels surrounding the locations of the 4 thermoprobes were not different at rest versus in motion (4.3 vs. $4.8, P=0.82$ ).

The dimensions of the area with $\mathrm{T} \geq 50^{\circ} \mathrm{C}$ corresponded best with the dimensions of macroscopical coagulation with a proportional correlation (all $r \geq 0.94$ with all $P \leq 0.017$ ). 

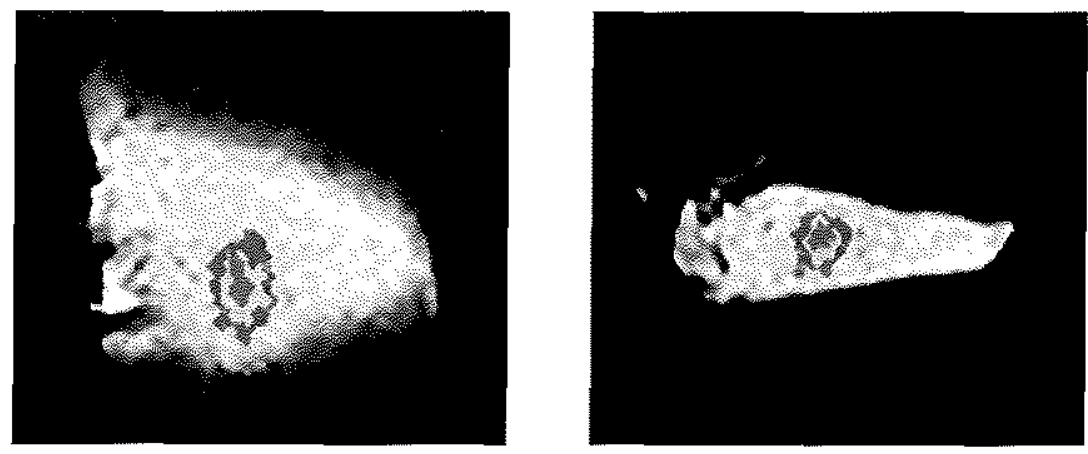

A

B

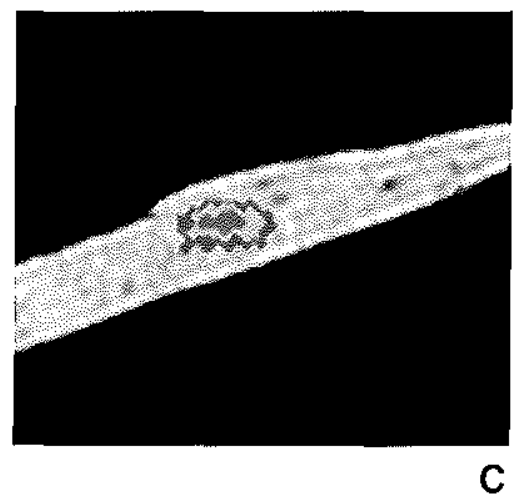

Figure 9.3 Transversal (A), coronal (B) and sagital (C) slice of liver lobe (ex vivo) scanned in dynamic series during $\mathrm{LCC}$ with $5 \mathrm{~W}$ for 6 minutes $(2160 \mathrm{~J})$. Colour overlays, over baseline modulus image, represent temperature increase compared to baseline $\left(32^{\circ} \mathrm{C}\right.$ in this particular case): $+8^{\circ} \mathrm{C}$ (blue), $+18^{\circ} \mathrm{C}$ (yellow) and $+28^{\circ} \mathrm{C}$ (red). 
Table 9.1 Deviation per minute $\left({ }^{\circ} \mathrm{C} \mathrm{min}^{-1}\right)$ of MR temperatures, determined with proton resonance frequency mapping, from true temperature, measured with interstitial thermoprobes. Experiments monitored the cooling of heated gel and temperature increase during ILC in ex vivo liver tissue.

\begin{tabular}{|c|c|c|c|c|}
\hline & \multicolumn{2}{|c|}{ Mean $\triangle \mathrm{T}\left({ }^{\circ} \mathrm{C} \min ^{-1}\right)$} & \multicolumn{2}{|c|}{ Mean $\Delta \mathrm{T}_{\text {corrected }}\left({ }^{\circ} \mathrm{C} \min ^{-1}\right)$} \\
\hline & At rest & In motion & At rest & In motion \\
\hline Gel & $0.06(0.12)$ & $0.31(0.32) *$ & $-0.15(0.28)$ & $-0.278(0.28) *$ \\
\hline Liver & $0.19(0.29)$ & $0.14(0.39) * *$ & $-0.04(0.34)$ & $-0.01(0.34) * *$ \\
\hline
\end{tabular}

Notes: Data are mean with standard deviation of 5 experimental results. ${ }^{*} P<0.05$ compared to at rest for similar MR temperature calculation, see text for exact $P$ values. ${ }^{*} P>0.05$ compared to at rest for similar modus of MR temperature calculation, see text for exact $P$ values.

\section{In vivo studies}

Two of 6 laser applications were terminated due to MR software failure and breakdown of the connecting laser fibre, leaving 4 experiments for analysis. Diameter of the induced spherical coagulation damage varied between 5.1 and $5.7 \mathrm{~cm}$. In the first two experiments assessment of temperature induced damage was impossible due to massive artefacts. Whilst no obvious heating around the laser fibre was observed, isolated pixels throughout the entire scanned planes showed temperature increase. In the latter 2 experiments the MRI scanning protocol was modified 1) a circular surface coil was applied to the liver above the area of coagulation, 2) scanning was not only triggered at inspiration but also at the beginning of the cardiac cycle and 3) anti-aliasing and noise filtering was applied more rigorously. The resulting real-time registrations corresponded better with the macroscopic damage and thermoprobe readouts. Insufficient data were collected for a quantitative verdict. 


\subsection{DISCUSSION}

In this study, we investigated whether simulated respiration motion affects the accuracy of MR phaseshift mapping for temperature measurements during local heat application. Accuracy was not diminished when the movement of respiration was introduced in the liver model whereas a significant decline was found in the gel model. Various modifications to the scanning protocol were necessary for satisfactory results in pigs. In addition it was found that MR-assessed temperature drifted away from real temperature in time. This drift was minimal; $0.5^{\circ} \mathrm{C}$ decrease in accuracy per minute in gel for a $95 \%$ confidence interval. For moving gel-filled syringes accuracy was significantly

less $\left(1.2^{\circ} \mathrm{C} \mathrm{min}-1\right)$, as well as moving liver $\left(1.1^{\circ} \mathrm{C} \mathrm{min}{ }^{-1}\right)$. Still, tissue temperature could be estimated after 6 minutes of interstitial laser application in liver with a $95 \%$ certainty of $\pm 3.5^{\circ} \mathrm{C}$.

Ideally, real-time monitoring of ILC should provide information about the extent of coagulation in relation to the anatomy of the liver during treatment: The intervention may be prolonged to ensure complete coagulation of the entire tumour or terminated to prevent damage to vital structures. To realise on-line monitoring of laser treatment, the thermal imaging potential of magnetic resonance has been considered before (Parker et al, 1983; Dickinson et al, 1986). Early research focused on the change in image contrast due to the change in spin-lattice time, or T1, of water (Lewa and Majeska, 1980). Because of the complicated temperature dependence of T1 (Bottomley et al, 1984; Young et al, 1994a) and the tissue dependence of T1 (Parker et al, 1983; Bottomley et al, 1984; Dickinson et al, 1986) current status of $\mathrm{T} 1$ weighted monitoring of heat deposition in tissue does not represent adequate thermometry. As an alternative, determination of the molecular diffusion has been proposed for temperature measurements based on a direct relationship between temperature and several diffusion coefficients quantifying the thermal Brownian motion (Le Bihan et al, 1989). Long acquisition times needed, up to 4 minutes, make the technique unsuitable for a rapid process as ILC and particularly susceptible to motion induced artefacts (Clegg et al, 1995).

In the present study, both in gel and in liver tissue it was found that accuracy of MR temperature measurements drifted away in time from the temperature measured by thermoprobes (Figure 9.2). This has also been described previously with the proton-resonance frequency method (De Poorter et al, 1994; De Poorter et al, 1995; Kahn et al, 1997; Sinha et al, 1997; Vitkin et al, 1997) and was attributed to both drift of the $\mathrm{B}_{0}$ field and field disturbances caused by the measurements themselves. However, correction for temperature-independent phase-shifts, by subtraction of the phase shift in reference locations counterbalanced this tendency (De Poorter et al, 1994; Sinha et al, 1997). In contrast, in the 
present study "in-experiment" calibration did not improve in more accuracy for gel, whereas for ex vivo liver only a modest improvement was found by using the data corrected for phase shifts at a reference location. This difference may be explained by 1) our use of models in which large temperature changes were studied over a small time frame (Lam and Webb, 1998) and 2) the use of one reference location instead of multiple sample points to correct for spatial imhomogeneities of the magnetic field (De Poorter et al, 1994). Calibration during treatment has also been suggested by combining phase with modulus information (Cline et al, 1995; Steiner et al, 1997) and by integrating diffusion-based or spectroscopic temperature measurements with the fast proton-resonance frequency shift method (De Poorter et al, 1994; Kuroda et al, 1998b). Differences in the accuracy between the gel and liver experiments may be explained by signal decrease in the coagulated area (De Poorter et al, 1994). Apart from the already mentioned sources of bias $\left(B_{0}\right.$ inhomogencities, magnetic field inhomogeneities, relocation induced artefacts), fat induced susceptibility artefacts have been suggested as a mechanism for uncertainty with phase shift mapping (de Zwart et al, 1996). A recent series of experiments by de Zwart et al. showed that lipid suppression indeed improved the precision of the temperature estimates (1999). Aside from the observed drift over time, accuracy was consistent with other recent in vitro (Stollberger et al, 1998) and ex vivo studies (Moriarty et al, 1998).
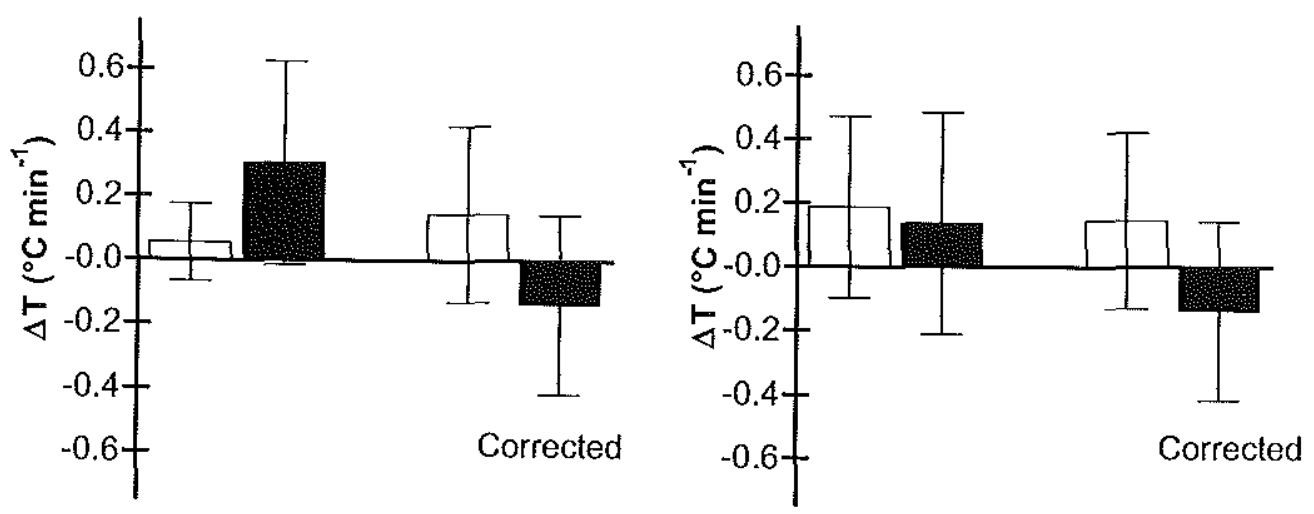

Figure 9.4 Accuracy of MR thermometry $\left(\Delta \mathrm{T} \min ^{-1}\right)$ in gel filled syringes (left) with mean of the scanned area and dispersion within this area at rest (white) and in motion (black). Corrected data: phase shifts in syringe at room temperature subtracted. Right: During laser application in ex vivo porcine liver at rest (white) and in motion (grey). Corrected data: phase shifts at remote position in liver subtracted. Data are means of 5 experimental results and brackets indicate standard deviation. 
From chapter 8 it was leamed that protein denaturation and tissue coagulation occur at temperatures between $50^{\circ} \mathrm{C}$ and $60^{\circ} \mathrm{C}$. In liver, the area of coagulation was clearly underestimated by the corresponding dimensions of MR isotherms at $60^{\circ} \mathrm{C}$ as compared with the macroscopy. Part of this discrepancy may be caused by a different slice angle of the MR plane compared to the plane of dissection of the liver. Furthermore, point precision of the temperature at several anatomical points is more important than isotherms or colour overlays. To confirm tissue coagulation, recently a postintervention $\mathrm{T} 2$ weighted scan has been suggested based on of the irreversible coagulation dependence of T2 (Graham et al, 1998). This type of scan may be an addition to MR-thermometry by phase-shift mapping doring laser treatment.

The experiments have been conducted in a model without tissue perfusion. Therefore, confirmation of these results in a preclinical in vivo animal model is mandatory. Although chemical shift measurements are relative insensitive to tissue properties (McFall et al, 1996; Peters et al, 1996), the blood perfusion of the hepatic parenchyma will make a major difference. Likely, our practice to occlude portal flow to the liver during laser application to increase laser capacity (chapters 5 and 6), diminishes the importance of this factor. In addition, liver motion in the body is more complex than the simulated motion in the single horizontal plane in this study (Jackson et al, 1993; Choe et al, 1997; Wilson et al, 1998). As pleaded before (Kettenbach et al, 1997), general anaesthesia with mechanical ventilation during laser treatment may be necessary for a constant motion pattern of the scanned region. Ventilation allows a stop for several seconds at expiration and inspiration to complete scanning of all slices at exactly the same location. Moreover, the deep analgesia prevents patient discomfort and the motion as the result of that. Even high frequency jet-ventilation with only minimal diaphragmal and hepatic movement (Sykes, 1989; Aloy et al, 1996) may be considered to minimise the influence of respiration induced artefacts.

In conclusion, it was found that tissue temperature in a homogeneous stationary medium can be estimated with a very high precision using phase-shift mapping by magnetic resonance. During laser application in ex vivo liver, it was shown that the accuracy is not hampered by (simulated) respiratory motion. The accuracy of the temperature estimates justifies further pre-clinical studies. 
, 


\section{Discussion and considerations for clinical application}

\subsection{INTRODUCTION}

Interstitial laser coagulation (ILC) is one of the modalities under investigation as an alternative for surgical resection in the treatment of patients with hepatocellular carcinoma or metastases of colorectal carcinoma. As for the other local modalities, the current status of ILC with Nd:YAG laser light does not yet ensure complete irradication in largers tumours. For ILC this is larger than approximately $2.5 \mathrm{~cm}$. The aim of the studies in this thesis was to optimise ILC. In chapter 1 an overview of $\amalg \mathrm{C}$ was given and the points of application for further improvement were discussed. They can be categorised in two items: 1) increase in the volume of coagulation and 2) improvements in the monitoring of the laser-induced tissue changes. Studies to both subjects have been conducted.

\subsection{INCREASING LESIONS SIZE}

Originally bare-tip fibres, fibres of which the coating at the distal end was stripped, were used in ILC as they are easy and cheap to manufacture. Furthermore it was suggested that such a point light source produces a larger volume of coagulation necrosis than a distributed light source with the same laser power and exposure time. The traditional bare-tip fibre was compared with a newly developed fibre with a tip from which the light was diffused over the length of a cylindrical tip. The volumes of coagulation produced by the cylindrical diffuser-tip were significantly larger and more predictable. Seven Watts during 9 minutes resulted in lesions with a length of $3.6 \mathrm{~cm}$ and a diameter of $2.3 \mathrm{~cm}$. Also less inter-lesion variation was found with the diffusing light source. An additional advantage of diffusing tips is the possibility to adapt the length of the tip to tumour diameter.

Still, the volume of coagulation produced with such a diffusing tip fibre was insufficient to treat large tumours. Simultaneous application of 4 fibres was investigated using a newly developed beamsplitting device, enabling the even distribution of a single laser beam over 4 laser fibres. 
Earlier beamsplitters often required cooling and lacked from an uneven distribution over the 4 channels. A synergistic effect, as predicted in mathematical models, was found between the 4 fibres. Spherical lesions with a volume up to $44.5 \mathrm{~cm}^{3}$ with a diameter of $5 \mathrm{~cm}$ were produced in $e x$ vivo porcine liver. The volume of coagulation depended on the mutual fibre distance, illustrated by the finding that smaller distances between the fibres resulted in smaller lesions with central carbonisation, whereas larger distances resulted in 4 separate zones of coagulation.

During the studies, performed in this thesis, a new light delivery device was proposed to increase to the volume of coagulation with a single applicator (Vogl et al, 1998). The laser fibre is introduced in the tissue within a closed catheter system, transparent for laser light. The catheter is irrigated with water during laser treatment. By temperature reduction at the surface of the catheter, exposition time can be prolonged without the risk of carbonisation and fibre destruction. Lesions up to $5.5 \mathrm{~cm}$ in diameter can be created when used in combination with a diffusing tip of $5 \mathrm{~cm}$ length. The insertion of 1 such fibre into a tumour is also more practical and precise than the positioning of four fibres at an equal depth and discrete mutual distance. Especially in percutaneous application this may be critical. A small deviation from linearity of the insertion tract can cause either diverging fibres with insufficient tissue coagulation or converging channels with an accumulation of light and heat with subsequent tissue carbonisation and fibre breakdown. In pigs, in which the ILC process was monitored with MRI, the water-cooled catheter was used with $20 \mathrm{~W}$ during 12 minutes. The diameter of the induced zone of coagulation varied between 5.1 and $5.7 \mathrm{~cm}$. In our clinical protocol the water-cooled catheter is now preferred for tumours larger than $2 \mathrm{~cm}$ in diameter.

In computer simulations (Semelka and Ascher, 1993) and mathematical modelling (Whelan et al, 1995) a four- to five- fold increase of the area of coagulation was predicted for laser application in liver tissue without blood flow and thus with minimal cooling. Although it had been observed that viable cells remained intact surrounding larger vessels after ILC, the magnitude of this effect was not yet appreciated in animal studies. Two factors may explain this: 1) the small volume of coagulation that could be produced with the available light delivery equipment and 2) the relatively small size of previous animal models.

ILC with portal inflow occlusion was differentiated from complete inflow occlusion (arterial and portal) and physiological flow through the liver. Occlusion of the portal flow substantially increased the area of themal necrosis. In this way intra-hepatic lesions of $4.5 \mathrm{~cm}$ in diametcr could be produced in pig liver in vivo. Additional clamping of the hepatic artery did not increase the volume of 
coagulation. Thus in the normal liver of pigs, portal inflow occlusion suffices to overcome the cooling effect of the blood flow.

With the knowledge of the vascularisation of hepatic tumours, these findings can be extrapolated to the human situation. Although hepatic metastases are mainly supplied by the arterial branch (Breedis and Young, 1954; Strohmeyer et al, 1987), clamping of both hepatic artery and portal vein is unlikely to be needed in the clinical situation. The poorly organised vascular bed in the fumour will probably cause only minimal tissue cooling and thus minimal lesion reduction (Peterson, 1979). Particularly in the often ischaemic centre of the tumour little extra effect can be expected from eliminating the arterial flow. The tumour, however, is surrounded by very well perfused liver parenchyma, in which the cooling effect is exerted by the portal flow (Strohmeyer et al, 1987; Archer and Gray, 1989). Therefore, eliminating portal flow should enable the destruction of the vital rim of the tumour and a margin of normal liver tissue. For $\mathrm{LC}$ of primary liver cancer, portal inflow occlusion may not be needed in a cirrhotic liver, as the portal flow is often even retrograde (Taourel et al, 1998). In this sifuation the magnitude of the arterial flow should be considered as a potential cooling source.

\subsection{LASER TISSUE INTERACTION AND MONITORING}

The possibility of larger volumes of coagulation also necessitate more knowledge on the laser-tissue interaction and real-time monitoring. The cooling effect of the blood flow not only reduces the volume of coagulation, it also protects the vascular wall from the thermal damage. Potential differences between the effects of $\Pi \mathrm{C}$ at central versus a peripheral location in the liver were investigated in a porcine model with portal inflow occlusion. Large vessels neighbouring to central lesions were always intact. Apparently the vessel wall is resistant to hyperthermia without the convection of heat by the blood flow. An interesting observation in the follow-up after ILC was that central lesions were always smaller than peripheral lesions. Probably volumes of induced coagulation in the central group were initially smaller. As central locations are surrounded by more liver parenchyma, the produced heat is convected and distributed over a larger volume, resulting in a smaller volume of coagulated tissue. This observation emphasises the importance of monitoring the laser-induced effects during treatment.

The lethality of ILC was investigated in human hepatic metastases of patients. Both a direct marker of cellular metabolism as well as an indicator of DNA integrity were studied in resection specimens with colorectal metastases subjected to ILC. It was found that no vital cells remain within the 
macroscopically coagulated whitish tumour. Furthermore there was no significant difference in the magnitude of the effect between normal porcine liver and colorectal carcinoma metastasised to the liver. Both conclusions are valid provided the portal flow is occluded. This permits the extrapolation of conclusions made from experiments in ex vivo porcine normal liver to clinical application.

As a first step to magnetic resonance monitoring the exact temperature at which coagulation occurs was investigated. Rat syngenic tumour samples were heated ex vivo at 4 different temperatures and 4 different exposure times. Three methods were used to assess cell viability. In agreement with studies on dissolved proteins we found that for solid tumours the temperature range resulting in sufficient tissue coagulation for cell death is between $50^{\circ} \mathrm{C}$ and $60^{\circ} \mathrm{C}$.

Using the threshold of $60^{\circ} \mathrm{C}$ we compared the MR thermometry results with macroscopic coagulation in ex vivo porcine liver after calibrating the MR thermometry in homogeneously heated gel samples. We found that the accuracy of the temperatures measurements decreased during the laser application. Correction for variation at a thermostabile reference location, thus with subtraction of temperature independent factors, did not enhance agreement. Temperatures could be estimated with a $95 \%$ certainty of $\pm 3.5^{\circ} \mathrm{C}$ after 6 minutes of laser application. As the used MRI method, MR phase-shift mapping, is a subtraction technique it is prone to the influence of abdominal respiratory movement. Additionally we did not find that accuracy was diminished when respiration was introduced into the models. In a pilot study MR thermometry was also investigated in pigs. Various factors necessitated adaptation of the scanning protocol and current investigations focus on optimising the computer algorithm to obtain the same accuracy as in the ex vivo experiments.

In a recent paper, 4 different magnetic resonance methods were compared and it was concluded that the method we used was preferable to other available methods (Wlodarczyk et al, 1999); MR phase-shift mapping is fast, accurate and can be performed with standard MR machines . But multiple factors interact in the complicated field of MR-themometry and extensive research will be necessary to create a reliable thermometer with indefinite sample points.

\subsection{FUTURE PERSPECTIVES AND CLINICAL APPLICATION}

Currently, surgical resection is the therapy of choice for colorectal liver metastases and primary liver cancer and should be performed whenever technically and medically feasible. Various local modalities are investigated for solid tumours within parenchymal organs. When with such a local treatment complete destruction can be achieved, the impact on patient survival may be comparable 112 
to surgical resection whilst the accompanied destruction of normal hepatic tissue is minimised. The crux will be to ensure that coagulation completely includes the tumour at stake, thus to achieve a local radical effect. Apart from the factors investigated in this thesis other aspects need to be considered.

\section{Procedure}

ILC can be performed percutaneously under intravenous sedation and analgesia. This enables the procedure to be performed within a short stay hospital visit with 24-hr post treatment observation. On the other hand general anaesthesia with artificial ventilation results in better respiration control for e.g. respiratory triggered MRI scanning; it also prevents patient discomfort and movement during treatment and makes vascular inflow occlusion better tolerable. Both in our experience and as reported by others percutaneous transhepatic catherisation and balloon occlusion of the portal vein can be performed safely by an experienced interventional radiologist (Funaki et al, 1995; Waiser et al, 1998; Heisterkamp et al, 1999). For lesions high in the hepatic dome, transthoracic and transdiaphragmatic fibre insertion may be necessary. For lesions located in the left hemi liver a subcostal route is feasible whereas for right located tumours insertion in the intercostal space should be considered to avoid hilar structures, especially when inserting multiple fibres.

Percutaneous treatment makes repeated treatment feasible. An advantage of an open or laparoscopic approach (Germer et al, 1997; Klotz et al, 1997) may be a more reliable fibre positioning under direct view, resulting in complete tumour destruction in a single treatment session. Also peritoneal deposits, making the laser treatment pointless, can be detected at laparotomy or laparoscopy. In pigs, laparoscopic $\amalg C$ has been combined with real-time MRI monitoring in an open interventional MRI (Klotz et al, 1997). In this way the preciseness of positioning under direct view is combined with reliable interstitial monitoring. Currently patient access is limited in high strength MRI machines; progress in this field and the development of MR compatible equipment promises future high-strength MRI which allow interventions to be performed from all sides (Gould and Darzi, 1998; Hinks et al, 1998).

Future laser application may be designed as depicted in Figure 10.1. In a computer simulation prior to treatment, areas of interest are indicated 1) the area to be heated above the critical isotherm for tissue coagulation: tumour plus a circumferential border of hepatic parenchyma, 2) anatomical landmarks to which damage should be avoided and 3) the type of fibres and length of the tip with the laser output. The patient is in the MR with the fibres inserted and connected to a beamsplitting 
device fed by the laser. During $\Pi \mathrm{LC}$, output from the MR, generated in real time, is interpreted and compared to treatment planning. These data are used to adapt the laser parameters by steering of the beamsplitter output. In this way both complete tumour plus a surrounding margin of hepatic tissue destruction as well as control of the themal process is ensured to prevent damage to vital hepatic structures. Although fiction now, advances might enable such a treatment in the near future.

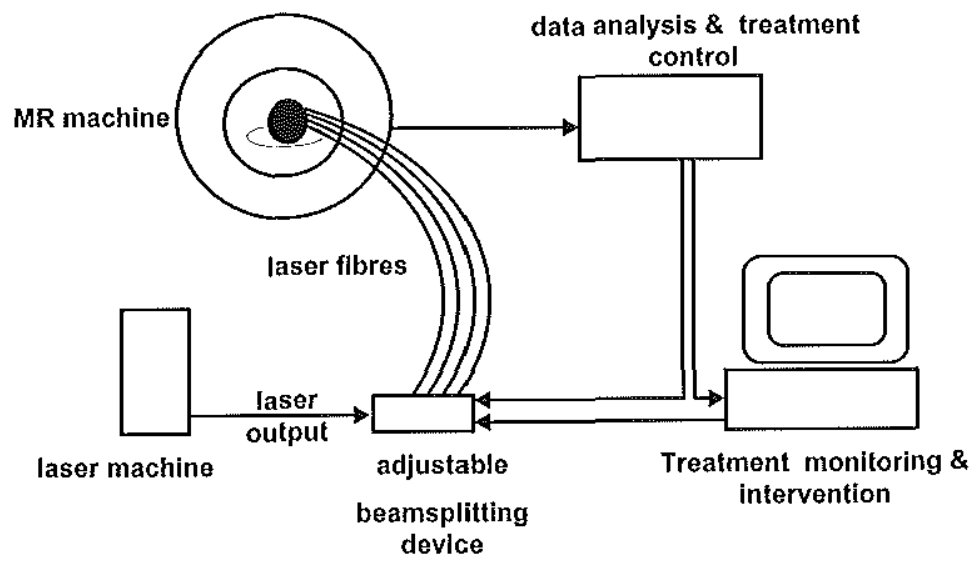

Figure 10.1 Schematic drawing of a future MRI guided interstitial laser coagulation.

\section{Combination with chemotherapy}

It is obvious that a local treatment such as $\amalg C$ results in local control only and does not affect the systemic dissemination of the disease. Thus, although extrahepatic disease is excluded at time of laser treatment, "recurrent" disease may develop elsewhere during follow up, apart from local recurrence in an incompletely treated tumour. In up to two-thirds of the patients undergoing partial liver resection for colorectal metastases, new tumours will be diagnosed during follow-up (Holm et al, 1989; Steele, Jr. 1996). In patients with hepatocellular carcinoma in a cirrhotic liver the chance of new tumour development is a certain 100\%. Currently, it is investigated in multi-centre trials whether there is a benefit from adjuvant therapy after hepatic resection of colorectal metastases. Post ILC, chemotherapy may then also be applicable for laser treated patients. Indeed, Bown and colleagues have noted in a retrospective studied cohort a remarkable increase in survival for 114 
patients treated with systemic chemotherapy in addition to ILC (personal communication, 1996).

\section{Combining surgery with ILC}

Combining a local modality with surgical resection appears attractive as this increases the pool of surgical candidates. In studies on cryosurgery up to $50 \%$ of the patients are treated with such a combined approach (Ravikumar et al, 1991b; Weaver et al, 1995; Adam et al, 1997; Hewitt et al, 1998). In our opinion, this is disputable: hepatic resection is considered to be justified only when a radical resection is feasible based on preoperative and peroperative imaging findings. Relying on a local modality with unproved benefit in patients with irresectable tumours, to "justify" the simultaneous resection, seems not appropriate. One might even argue that when relying on the local modality, the resection should be withheld in those patients and all the metastases should be controlled locally. Until the benefits of the local modality have been shown convincingly, we feel that combining resection with an experimental method is unethical. When this evidence is available, this should then prompt studies comparing surgery with the local modality for patients with resectable metastases.

\section{Rotterdam clinical experience}

The investigations described in this thesis were integrated in a clinical protocol. In three subsequent phases the role of ILC in the management of patients with secondary or primary hepatic tumours is investigated. In the first phase the feasibility, complications and initial tumour response are determined in 10 patients with irresectable hepatic tumours. Recently this phase of the study has been completed.

In 10 patients 14 hepatic tumours ( $7 \mathrm{HCC}, 7 \mathrm{HM},>2.5 \mathrm{~cm}$ in diameter) have been lased; 2 at laparotomy and 9 percutaneously. Reasons for ineligibility for surgery were anatomic limitations ( $n$ $=3$ patients), Child-B cirrhosis $(n=5)$ or comorbidity $(n=2)$. ILC was performed under general anaesthesia at laparotomy or percutaneously with predefined laser parameters based on tumour diameter. Percutaneous treatment was performed in the angiography suite by a radiologist. During ILC only, hepatic blood inflow was occluded, either by peroperative clamping or by percutaneous transluminal occlusion with a balloon catheter; for HCC the hepatic artery and for HM the portal vein was occluded.

In 4 patients minor complications were observed; pain at insertion site ( $n=4$ patients), diaphragmal irritation with shoulder tip pain and pleural exudate requiring drainage $(n=1)$, inguinal hematoma 
$(n=1)$ and fever without a focus for infection $(n=1)$. In three patients discharge was delayed by the minor accompanying effects of ILC. The remaining 6 out of 9 patients treated percutaneously were discharged 24 hours after ILC. One day post treatment response rate was determined using triphasic spiral CT. Uptake of contrast by the tumour is used to classify the amount of coagulation within the tumour (Amin et al, 1993c). Nine out of 14 (65\%) tumours were completely avascular, more than $50 \%$ of the tumour volume was avascular in 4 tumours and in 1 tumour avascularity was induced in $<50 \%$ of the tumour volume. Failures to complete coagulation was independent of tumour diameter and attributable to laser fibre breakdown $(n=2)$, calcified tumour mass precluding central fibre positioning $(n=1)$, and an unexpected large tumour diameter diagnosed during ultrasound guided fibre positioning $(n=1)$. After introduction of the water-cooled fibre catheter fibre breakdown did not occur anymore. In the last seven treatment sessions complete coagulation was always achieved.

These results indicate that ILC with vascular inflow occlsusion is a safe and feasible technique which can be performed percutaneously in a short-stay admission. Complete destruction was realised in $65 \%$ of the tumours with a steep learning curve. Supported by these findings we have entered the second phase in which the rate of local control is determined 1 year after $\Pi L C$ with complete response (Amin grade 1). 


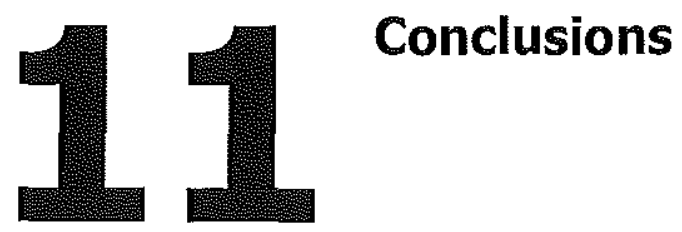

Interstitial laser coagulation (ILC) is a suitable technique for the local destruction of solid tumours within parenchymal organs such as hepatic metastases of colorectal carcinoma or hepatocellular carcinoma. From clinical series it was concluded that ILC is a safe technique but that several improvements in the technique were mandatory to allow treatment of tumours larger than approximately $2.5 \mathrm{~cm}$ in diameter. First the volume of coagulation that can be induced in a single application should be increased secondly the laser-tissue interaction should be better understood in order to realise real-time monitoring of the laser-induced effects. Investigations to both aspects were addressed in this thesis with the following conclusions:

1) Interstitial laser coagulation is now also successfully applicable to tumours larger than $2.5 \mathrm{~cm}$ in diameter by preventing carbonisation with the use of light emitting fibres with a cylindrical diffusing tip. The simultaneous application of 4 diffusing fibres results in an additional synergistic effect between the 4 fibres. The same volume of coagulation can be achieved with a single water-cooled catheter. The largest volume of coagulation which can be created with such a single application is $5.5 \mathrm{~cm}$.

2) In vivo these modifications will only be effective when the major vascular inflow is occluded during treatment. Flow occlusion does not damage neighbouring structures and is safe to vital structures within the liver. Flow occlusion warrants complete cell death within the area of macroscopic coagulation.

3) In interstitial laser coagulation the critical isotherm for sufficient protein denaturation for complete cell death is between $50^{\circ} \mathrm{C}$ and $60^{\circ} \mathrm{C}$. With magnetic-resonance chemical-shift measurements this temperature can be estimated with a certainty of $\pm 3.5^{\circ} \mathrm{C}$ during ILC in ex 
vivo porcine liver. The preciseness is not affected by respiration induced motion, but further studies are indicated to extrapolate the accuracy into the in vivo situation.

In conclusion it is demonstrated that complete coagulation can be achieved in volumes to $5.5 \mathrm{~cm}$ in diameter. In our view, the results of the pre-clinical studies in this thesis justify clinical evaluation of benefit of portal inflow occlusion, multiple fibre- and water-cooled fibre- application. We hypothesise that these modifications result in a higher proportion of successful treatment in a single session for larger tumours. If the accuracy and efficacy of ILC has been established unequivocally, comparing ILC with surgical resection will be justified. 


\section{APPENDICES}





\section{Bibliography}

Adam R, Akpinar E, Johann M, Kunstlinger F, Majno P, Bismuth H. (1997) Place of cryosurgery in the treatment of malignant liver tumors. Anm. Surg. 225:39-50.

Aloy A, Donner E, Lanzenberger E, Kashanipour A. (1996) Low-frequency and high-frequency jet ventilation technical basics and special considerations for clinical application in various areas (Review). Acta Anaesthesiol. Scand. Suppl. 109:147-149.

Amin Z, Bown SG, Lees WR. (1993a) Local treatment of colorectal liver metastases: a comparison of interstitial laser photocoagulation (ILP) and percutaneous alcohol injection (PAI). Clin. Radiol. 48:166-171.

Amin Z, Buonacorssi G, Mills T, Harries SA, Lees WR, Bown SG. (1993b) Interstitial laser photocoagulation: Evaluation of a $1320 \mathrm{~nm}$ Nd:YAG and a $805 \mathrm{~nm}$ Diode Laser: the significance of charring and the value of pre-charring the fibre-tip. Lasers Med Sci. 8:113-120.

Amin Z, Donald JJ, Masters A, Kant R, Steger AC, Bown SG, Lees WR. (1993c) Hepatic metastases: interstitial laser photocoagulation with real-time US monitoring and dynamic CT evaluation of treatment. Radiology. 187:339-347.

Amin Z, Harries SA, Lees WR, Bown SG. (1993d) Interstitial tumour photocoagulation. Endosc. Surg. Allied, Techol. 1:224-229.

Amin Z, Thurrell W, Spencer GM, Harries SA, Grant WE, Bown SG, Lees WR. (1993e) Computed tomography-pathologic assessment of laser-induced necrosis in rat liver. Invest. Radiol. 28:1148-1154.

Archer SG and Gray BN. (1989) Vascularization of small liver metastases. Br J Surg. 76:545-548.

Ascher PW. (1996) Personal communication. Europan Biomedical Optics Week, Vienna, September 8, 1996. Attwood SE, Caldwell MT, Marks P, McDermott M, Stephens RB. (1994) Adhesions after laparoscopic inguinal hernia repair. A comparison of extra versus intra peritoneal placement of a polypropylene mesh in an animal model. Strg Endosc. 8:777-780.

Bartolozzi C and Lencioni R. (1996) Ethanol injection for the treatment of hepatic tumours (Review). Eur. Radiol. 6:682-696.

Boni RA, Sulser T, Jochum W, Romanowski B, Debatin JF, Krestin GP. (1997) Laser ablation-induced changes in the prostate: findings at endorectal MR imaging with histologic correlation. Radiology. 202:232236.

Bosman S, Phoa SS, Bosma A, van Gemert MJ. (1991) Effect of percutaneous interstitial thermal laser on normal liver of pigs: sonographic and histopathological correlations. Br. J. Surg. 78:572-575.

Bosman S. (1993) Thermal effects of laser light on tissue structure. Thesis, University of Amsterdam.

Bottomley PA, Forster TH, Argersinger RE, Pfeifer LM. (1984) A review of normal tissue hydrogen NMR relaxation times and relaxation mechanisms from 1-100Mhz: dependency on tissue type. Med Phys. 11:425448.

Bown SG. (1983) Phototherapy in fumors. World J Surg. 7:700-709.

Bown SG. (1996) Personal communication. Annual symposium Dutch Medical Laser Association. Topics of laser application in general surgery and gynaecology. March 22, 1996. Rotterdam.

Breedis C and Young C. (1954) The blood supply of neoplasms in the liver. Am J Pathol. 30:969-985.

Carlsson G, Gullberg B, Hafstrom L. (1983) Estimation of liver tumor volume using different formulas -an experimental study in rats. J Cancer Res Clin Oncol. 105:20-23.

Carter DL, MacFall JR, Clegg ST, Wan X, Prescott DM, Charles HC, Samulski TV. (1998) Magnetic resonance thermometry during hyperthermia for human high-grade sarcoma. Int. J. Radiat. Oncol. Biol. Phys. 40:815-822.

Castren-Persons M, Lipasti J, Puolakkainen P, Schroder T. (1992) Laser-induced hyperthermia: comparison of two different methods. Lasers Surg Med. 12:665-668.

Castren-Persons M, Schroder T, Lehtonen E. (1996) Sensitivity to Nd:YAG induced laserthermia is a celltype-specific feature not directly related to tumorigenic potential or proliferation rate. Lasers Surg Med. 18:420-428. 
Castro DJ, Saxton RE, Layfield LJ, Fetterman HR, Tartell PB, Robinson JD, To SY, Nishimura E, Lufkin RB. (1990) Interstitial laser phototherapy assisted by magnetic resonance imaging: a new technique for monitoring laser-tissue interaction. Laryngoscope. 100:541-547.

Centraal Bureau voor de Statistiek. (1998) Tijdreeksen Mens en Maatschappij. Kanker aan de spijsverteringsorganen. www.statline.cbs.nl.

Cheng KH and Hernandez M. (1992) Magnetic resonance diffusion imaging detects structural damage in biological tissues upon hyperthermia. Cancer Res. 52:6066-6073.

Choe KA, Smith RC, Wilkens K, Constable RT. (1997) Motion artifact in T2-weighted fast spin-echo images of the liver: effect on image contrast and reduction of artifact using respiratory triggering in normal volunteers. J. Magn. Reson. Imaging. 7:298-302.

Clegg ST, Das SK, Zhang Y, Macfall J, Fullar E, Samulski TV. (1995) Verification of a hyperthermia model method using MR thermometry. Int. J. Hyperthermia. 11:409-424.

Cline HE, Hynynen K, Schneider E, Hardy CJ, Maier SE, Watkins RD, Jolesz FA. (1995) Simultaneous magnetic resonance phase and magnitude temperature maps in muscle. In Proceedings of the International Society for Magnetic Resonance in Medicine. Third scientific meeting, p 1174 Society of Magnetic Resonance in Medicine: Berkeley, Ca.

Consensus Statement. (1997) Treatment of hepatic metastases from colorectal cancer. Journal of Gastrointest Surg. 1:396-397.

Crews KA, Kuhn JA, McCarthy TM, Fisher TL, Goldstein RM, Preskitt JT. (1997) Cryosurgical ablation of hepatic tumors. Am. J. Surg. 174:614-618.

Cromheecke M, de Jong KP, Hoekstra HJ. (1999) Current treatment for colorectal cancer metastatic to the liver. Eur. J. Surg. Oncol. 25:451-463.

Curley SA, Izzo F, Delrio P, Ellis LM, Granchi J, Vallone P, Fiore F, Pignata S, Daniele B, Cremona F. (1999) Radiofrequency ablation of unresectable primary and metastatic hepatic malignacies. Ann. Surg. 230:1-8.

Cuschieri A, Crosthwaite G, Shimi S, Pietrabissa A, Joypaul V, Tair I, Naziri W. (1995) Hepatic cryotherapy for liver tumors. Development and clinical evaluation of a high-efficiency insulated multineedle probe system for open and laparoscopic use. Surg Endosc. 9:483-489.

Dachman AH, McGehee JA, Beam TE, Burris JA, Powell DA. (1990) US-guided percutaneous laser ablation of liver tissue in a chronic pig model. Radiology. 176:129-133.

Davis M, Dowden J, Steger AC, Kapadia P, Whiting P. (1989) A mathematical model for interstitial laser treatment of tumours using four fibers. Lasers Med Sci. 4:41-53.

De Luca C. (1965) The use of trypsin for the determination of cellular viability. Exp. Cell Res, 40:186-188.

De Poorter J, de Wagter C, De Deene Y, Thomsen C, Achten E. (1994) The proton-resonance-frequencyshift method compared with molecular diffusion for quantative measurement of two-dimensional timedependent temperature distribution in a phantom. Joumal of Magnetic Resonance. series B 103:234-241.

De Poorter J, de Wagter C, De Deene Y, Thomsen C, Stahlberg F, Achten E. (1995) Noninvasive MRI Thermometry with the Proton Resonance Frequency (RPF) Method: In Vivo Results in Human Muscle. Magn Res Med. 33:74-81.

de Wit JN and Swinkels GA. (1980) A differential scanning calorimetric study of the thermal denaturation of bovine beta-lactoglobulin. Thermal behaviour at temperatures up to 100 degrees C. Biochim Biophys Acta. 624:40-50.

de Zwart JA, van Gelderen P, Kelly DJ, Moonen CTW. (1996) Fast magnetic-resonance temperature imaging. Joumal of Magnetic Resonance, series B. 112:86-90.

de Zwvart JA, Vimeux FC, Delalande C, Canioni P, Moonen CTW. (1999) Fast lipid-supressed MR temperature mapping with echo-shift gradient echo imaging and spectral-spatial excitation. Magn Res Med. 42:53-59.

Di Stasi M, Buscarini L, Livraghi T, Giorgio, Salmi A, De Sio I, Brunello F, Solmi L, Caturelli E, Magnolfi F, Caremani M, Filice C. (1997) Percutaneous ethanol injection in the treatment of hepatocellular carcinoma. Scand J Gastroenterol. 32:1168-1173.

Dickinson RJ, Hall AS, Hind AJ, Young IR. (1986) Measurement of changes in tissue temperature using MR imaging. J. Comput. Assist. Tomogr: 10:468-472. 
Dilley AV, Dy DY, Warlters A, Copeland S, Gillies AE, Morris RW, Gibb DB, Cook TA, Morris DL. (1993) Laboratory and animal model evaluation of the Cryotech LCS 2000 in hepatic cryotherapy. Cryobiology. 30:74-85.

Dowlatshahi K, Babich D, Bangert JD, Kluiber R. (1992a) Histologic evaluation of rat mammary tumor necrosis by interstitial Nd:YAG laser hyperthermia. Lasers Surg Med. 12:159-164.

Dowlatshahi K, Bhattacharya AK, Silver B, Matalon T, Williams JW. (1992b) Percutaneous interstitial laser therapy of a patient with recurrent hepatoma in a transplanted liver. Surgery. 112:603-606.

Dowlatshahi K, Saclarides T, Silver B, Fan M. (1995) Interstitial laser therapy of malignant liver tumors (Abstract). Lasers Surg Med. 18:18

El-Serag HB and Mason AC. (1999) Rising incidence of hepatocellular carcinoma in the United States. $N$. Engl. J. Med. 340:745-750.

Eriksson RA, Albrektsson T, Magnusson B. (1984) Assessment of bone viability after heat trauma. A histological, histochemical and vital microscopic study in the rabbit. Scandinavian joumal of plastic and reconstructive surgery. 18:261-268.

Filipponi F, Leoncini G, Campatelli A, Bagnolesi A, Perri G, Romagnoli P, Mosca F. (1995) Segmental organization of the pig liver: Anatomical basis of controlled partition for experimental grafting. European Surgical Research. 27:151-157.

Frank F and Hessel S. (1990) Technical prerequisites for the interstitial thermotherapy with the Nd:YAG laser. Lasermedizin. 1:36-40.

Fried MP, Morrisson PR, Hushek SG. (1996) Dynamic T1 weighted magnetic resonance imaging of interstitial laser photocoagulation in the liver: observations on in vivo temperature sensitivity. Lasers Surg Med.410-419.

Funaki B, Rosenblum JD, Leef JA, Alonso EM. (1995) Portal vein stenosis in children with segmental liver transplants: treatment with percutaneous transhepatic venoplasty. AJR. Am J. Roentgenol. 165:161-165.

Germer CT, Albrecht D, Roggan A, Isbert C, Buhr HJ. (1997) Experimental study of laparoscopic laserinduced thermotherapy for liver tumours. Br J Surg. 84:317-320.

Germer CT, Roggan A, Ritz JP, Isbert C, Albrecht D, Mulfer G, Buhr HJ. (1998) Optical properties of native and coagulated human liver tissue and liver metastases in the near infrared range. Lasers Surg Med. 23:194203.

Gewiese B, Beuthan J, Fobbe F, Stiller D, Muller G, Bose-Landgraf J, Wolf KJ, Deimling M. (1994) Magnetic resonance imaging-controlled laser-induced interstitial thermotherapy. Invest. Radiol. 29:345-351.

Gierada DS, Curtin JJ, Erickson SJ, Prost RW, Strandt JA, Goodman LR. (1995) Diaphragmatic motion: fast gradient-recalled-echo MR imaging in healthy subjects. Radiology. 194:879-884.

Gillams AR, Brookes J, Hare C, Bown SG, Taylor I, Ledermann J, Lees WR. (1997) Follow up of patients with metastatic liver lesions treated with interstitial laser therapy (Abstract). Br. J. Cancer: 76:31

Giovannini $M$ and Seitz JF. (1994) Ultrasound-guided percutaneous alcohol injection of small liver metastases. Results in 40 patients. Cancer. 73:294-297.

Goebel KR. (1994) Fundamentals of laser science. Acta Neurochirurgicae. 1994 (Suppl):20-33.

Gold R, Schmied M, Giegerich G, Breitschopf H, Hartung HP, Toyka KV, Lassmann H. (1994) Differentiation between cellular apoptosis and necrosis by the combined use of in situ tailing and nick translation techniques [see comments]. Lab. Invest. 71:219-225.

Gordon AD, Buonaccorsi G, Patel BL, Broadbent JAM, Thurell W, Bown SG. (1996) Interstitial laser photocoagulation of uterine leiomyoma. In Medical applications of lasers III, Proc of SPIE Vol 2623. Bown SG and Reidenbach HD(eds.) pp 218-221. SPIE, Bellingham.

Gould SW and Darzi A. (1998) The magnetic resonance operating theafre. Br J Surg. 84:595-597.

Graham SJ, Bronskill MJ, Henkelman RM. (1998) Time and temperature dependence of MR parameters during thermal congulation of ex vivo rabbit muscle. Magn Res Med. 39:198-203.

Gutman M and Fidler IJ. (1995) Biology of human colon cancer metastasis. World J Surg. 19:226-234.

Hahl J, Haapiainen R, Ovaska J, Puolakkainen P, Schroder T. (1990) Laser-induced hyperthermia in the treatment of liver tumors. Lasers Surg Med. 10:319-321.

Harries SA, Amin Z, Smith ME, Lees WR, Cooke J, Cook MG, Scurr JH, Kissin MW, Bown SG. (1994) Interstitial laser photocoagulation as a treatment for breast cancer. Br. J. Surg. 81:1617-1619. 
Harth T, Kahı T, Rassek M, Schwabe B, Schwarzmaier HJ, Lewin JS, Modder U. (1997) Determination of laser-induced temperature distributions using echo-shifted TurboFLASH. Magn. Reson. Med. 38:238-245. Hashimoto D, Takami M, Idezuki Y. (1985) In depth radiation therapy by YAG laser for malignant tumors of the liver under ultrasound imaging. Gastroenterology. 88:1663

Heisterkamp J, van Hillegersberg, de Man RA, Pattynama PMT, Brouwer JT, IJzermans JN. (1999) Selective hepatic inflow occlusion to enhance interstitial laser coagulation (ILC): Feasibility in 7 patients with hepatic tumours. Samenvatingen Voorjaarsvetgadering van de Nederlandse Vereniging voor Hepatologie.

Henkel TO, Niedergehtmann M, Alken P. (1996) Critical evaluation of laser-induced interstitial thermotherapy (LITT) performed on in vitro, in vivo and ex vivo models. In Medical applications of lasers III, Proc. of SPIE 2623. Bown SG and Reidenbach HD(eds.) pp 266-275. SPIE, Bellingham.

Herfarth C and Kallinowski F. (1997) Can clinical assessment predict a patient's prognosis after liver metastasis (Editorial). Ann. Strrg. 226:581

Hewitt PM, Dwerryhouse SJ, Zhao J, Morris DL. (1998) Multiple bilobar liver metastases: cryotherapy for residual lesions after liver resection. J. Strg. Oncol. 67:112-116.

Hill CR and ter Haar GR. (1995) Review article: high intensity focused ultrasound: potential for cancer treatment (Review). Br. J. Radiol. 68:1296-1303.

Hinks RS, Bronskill MJ, Kucharczyk W, Bernstein M, Collick BD, Henkelman RM. (1998) MR systems for image-guided therapy. JMRI. 8:19-25.

Holm A, Bradley E, Aldrete JS. (1989) Hepatic resection of metastasis from colorectal carcinoma. Morbidity, mortality, and pattern of recurrence. Ann. Surg. 209:428-434.

Hoye RC, Thomas LB, Riggle GC, Ketcham A. (1968) Effects of neodymium laser on normal liver and Vx2 carcinoma transplanted into the liver of experimental animals. J. Natl. Cancer Inst. 41:1071-1082.

Huang GT, Wang TH, Sheu JC, Daikuzono N, Sung JL, Wu MZ, Chen DS. (1991) Low-power laserthermia for the treatment of small hepatocellular carcinoma. Eur. J. Cancer. 27:1622-1627.

Hunter JG and Dixon JA. (1985) Lasers in cardiovascular surgery: current status. West. J Med. 142:506-510.

IJzermans JN and Bac D. (1997) Recent developments in screening, diagnosis and surgical treatment of hepatocellular cacrcinoma. Scand. J. Gastroenterol. 32:50-54.

Ikeda N, Hayashida O, Kameda H, Ito H, Matsuda T. (1994) Experimental study on thermal damage to normal dog brain. Imt. J. Hyperthermia. 10:553-561.

Ireland AP, Clark GW, DeMeester TR. (1997) Barrett's esophagus. The significance of p53 in clinical practice. Ann. Surg. 225:17-30.

Isbert CM, Germer CT, Albrecht D, Schilling A, Heiniche A, Roggan A, Muller G, Buhr HJ. (1997) Laserinduced thermotherapy (LITT) for the treatment of liver metastases- The correlation of Gd-DTPA-enhanced magnetic resonance in imaging (MRI) and histomorphological findings in the chronic model. In Lasers in Surgery: Advanced characteristics, therapeutics and systems VII, Anderson RR(ed) pp 543-553. SPIE: Bellingham.

Ishihara Y, Calderon A, Watanabe H, Okamoto K, Suzuki Y, Kuroda K. (1995) A precise and fast temperature mapping using water proton chemical shift. Magn. Reson. Med. 34:814-823.

Ivarsson K, Olsrud J, Sturesson C, Moller PH, Persson BR, Tranberg KG. (1998) Feedback interstitial diode laser $(805 \mathrm{~nm})$ thermotherapy system: ex vivo evaluation and mathemical modelling with one and fourfibers. Lasers Surg Med. 22:86-96.

Izzo F, Cremona F, Ruffolo F, Palaia R, Parisi V, Curley SA. (1998) Outcome of 67 patients with hepatocellular cancer detected during screening of 1125 patients with chronic hepatitis. Ann. Surg. 227:513518.

Jackson PC, Davies SC, Zananiri FV, Bean JP, Follett DH, Halliwell M, Wells PN. (1993) The development of equipment for the technical assessment of respiratory motion induced artefacts in MRI. British Joumal of Radiology. 66:132-139.

Johnson LB, Krebs TL, Van Echo D, Plotkin JS, Njoku M, Wong JJ, Daly BD, Kuo PC. (1997) Cytoablative therapy combined with resection and cryosurgery for limited bilobar hepatic colorectal metastases. Am. J. Sturg. 174:610-613.

Jolesz FA, Kahn T, Lufkin R. (1998) Genesis of interventional MRI (Editorial). JMRI. 8:2 
Jolesz FA and Blumenfeld SM. (1994) Interventional use of magnetic resonance imaging (Review). Magn. Reson. Q. 10:85-96.

Joly M. (1965) A physico-chemical approach to denaturation of proteins.

Kahn T, Harth T, Schwabe B, Schwarzmaier HJ, Modder U. (1997) MR tomographic temperature quantification at $1.5 \mathrm{~T}$ in vitro: a comparison of fast $\mathrm{T} 1$ maps and a phase-sensitive sequence. ROFO.:187193.

Kettenbach J, Silverman SG, Kuroda K, Nakajima Y, Zientara GI, Saiviroonporn P, Hata N, Morisson PR, Huskek SG, Black M, Kikinis R, Jolesz FA. (1997) Real-time monitoring and quantitative analysis of MRguided laser ablation. In Proceedings of the International Society for Magnetic Resonance in Medicine. Fifth scientific meeting, p 523 Society of Magnetic Resonance in Medicine: Berkeley, Ca.

King's Fund Forum. (1990) Cancer of the colon and rectum (consensus statement). Br. J. Surg. 77:10631065 .

Klotz HP, Flury R, Erhart P, Steiner P, Debatin JF, Uhlschmidt G, Largiader F. (1997) Magnetic resonanceguided laparoscopic interstitial laser therapy of the liver. Am. J. Surg. 174:448-451.

Kuroda K, Oshio K, Chung AH, Hynynen K, Jolesz FA. (1997) Temperature mapping using the water proton chemical shift: A chemical shift selective phase mapping method. Magn Res Med. 38:845-861.

Kuroda K, Chung AH, Hynynen K, Jolesz FA. (1998a) Calibration of water proton chemical shift with temperature for noninvasive temperature imaging during focused ultrasound surgery. JMRI. 8:175-181.

Kuroda K, Oshio K, Mulkern RV, Nakai T, Moriya T, Hynynen K, Jolesz FA. (1998b) Temperature mapping using water proton thermal shift: self referenced method with EPSI. In Proceedings of the International Society for Magnetic Resonance in Medicine. Sixth scientific meeting. Society of Magnetic Resonance in Medicine: Berkeley, Ca.

LaHaye M, Pow-Sang M, Orihuela E, Motamedi M. (1995) Effect of temperature and flow rate of irrigant on coagulation necrosis in canine prostate treated with neodymium:YAG laser. Joumal of Endourology. 9:155158.

Lam MM and Webb AG. (1998) Errors in temperature estination using MRI during rapid heating: a simulation study. In Proceedings of the International Society for Magnetic Resonance in Medicine. Sixth scientific meeting, Society of Magnetic Resonance in Medicine: Berkeley, Ca.

Larsson B, Astrom G, Einarsson S, Ryden L, Granberg-Ohman I, Lindhe BA. (1981) The possible risks of a copper and an inert intrauterine device situated in the abdominal cavity: an experimental study in pigs and dogs. Fertil. Steril. 36:229-231.

Le Bihan D, Delannoy J, Levin RL. (1989) Temperature mapping with MR imaging of molecular diffusion: application to hyperthermia. Radiology: 171:853-857.

Lee FT, Mahvi D, Chosy SG, Onik GM, Wong WS, Littrup PJ, Scanlan KA. (1997) Hepatic cryosurgery with intraoperative US guidance (review). Radiology, 202:624-632.

Leen E, Goldberg JA, Robertson J, Angerson WJ, Sutherland GR, Cooke TG, McArdle CS. (1993) Early detection of occult colorectal hepatic metastases using duplex colour Doppler sonography. $B r J$ Surg. 80:1249-1251.

Lepock JR, Frey HE, Heynen MP, Nishio J, Waters B, Ritchie KP, Kruuv J. (1990) Increased thermostability of thermotolerant CHL V79 cells as determined by differential scanning calorimetry. J. Cell Plyssiol. 142:628-634.

Lepock JR, Frey HE, Ritchie KP. (1993) Protein denaturation in intact hepatocytes and isolated cellular organelles during heat shock. $J$ Cell Biol. 122:1267-1276.

Lewa $C J$ and Majeska $Z$. (1980) Temperature realationships of proton-lattice relaxation time $\mathrm{T} 1$ in biological tissues. Bull Cancer (Paris). 67:525-530.

Livraghi T, Lazzaroni S, Pellicano S, Ravasi S, Torzilli G, Vettori C. (1993) Percutaneous ethanol injection of hepatic tumors: single-session therapy with general anesthesia. AJR. Am. J. Roentgenol. 161:1065-1069.

Livraghi T, Makuuchi M, Buscarini L. (1997) Diagnosis and treatment of hepatocellular carcinoma. 1:1-454. Lufkin RB. (1995) Interventional MR imaging [Editorial]. Radiology. 197: 16-18.

Malone DE, Wyman DR, DeNardi FG, McGrath FP, De Gara CJ, Wilson BC. (1994) Hepatic interstitial laser photocoagulation. An investigation of the relationship between acute thermal lesions and their sonographic images. Invest. Radiol. 29:915-921. 
Marquet RL, Westbroek DL, Jeekel J. (1984) Interferon treatment of a transplantable rat colon adenocarcinoma: importance of tumor site. Int. J. Cancer, 33:689-692.

Masters A, Steger AC, Bown SG. (1991) Role of interstitial therapy in the treatment of liver cancer. $B r . J$. Surg. 78:518-523.

Masters A and Bown SG. (1990) Interstitial laser hyperthermia in tumour therapy. Ann. Chir: Gynaecol. 79:244-251.

Masters A and Bown SG. (1992) Interstitial laser hyperthermia. Semin. Surg. Oncol. 8:242-249.

Matsumoto R, Selig AM, Colucci VM, Jolesz FA. (1992) Interstitial Nd:YAG laser ablation in normal rabbit liver: trial to maximize the size of laser-induced lesions. Lasers Sug Med. 12:650-658.

Matthewson K, Coleridge-Smith P, O'Sullivan JP, Northfield TC, Bown SG. (1987) Biological effects of intrahepatic neodymium:yttrium-aluminum-gamet laser photocoagulation in rats. Gastroenterology. 93:550557.

Matthewson K, Barr H, Tralau C, Bown SG. (1989) Low power interstitial Nd YAG laser photocoagulation: studies in a transplantable fibrosarcoma. Br. J. Surg. 76:378-381.

McCall Л, Jorgensen JO, Morris DL. (1996) Laparoscopic hepatic cryotherapy: a study of safety in rabbits. Surg Laparose. Endosc. 6:29-31.

McFall JR, Prescott DM, Charles HC, Samulski TV. (1996) 1H MRI phase thermometry in vivo in canine brain, muscle and tumor tissue. Med. Phys. 23:1775-1782.

Mesecke von Rheinbaben I, Roggan A, Muller GJ. (1996) In-vitro investigations to compare various wavelengths for faser-induced interstitial thermotherapy. In Laser Applications in Medicine and Dentistry (Proc SPIE 2922), Altsluler GB et al. (eds.) pp 334-341. SPIE: Bellingham.

Moller PH, Hannesson P, Ivarsson K, Olsrud J, Stenram U, Tranberg KG. (1997a) Interstitial laser thermotharapy in pig liver: effect of inflow occlusion on extent of necrosis and ultrasound image. HepatoGastroenterology: 44:1302-1311.

Moller PH, Ivarsson K, Stenram U, Radnell M, Tranberg KG. (1997b) Interstitial laser thermotherapy of adenocarcinoma transplanted into rat liver. Eur. J. Surg. 163:861-870.

Mordon S, Desmettre T, Devoisselle JM. (1995) Laser-induced release of liposome-encapsulated dye to monitor tissue temperature: a preliminary in vivo study. Lasers Surg Med. 16:246-252.

Moriarty JA, Chen JC, Purcell CM, Ang LC, Hinks RS, Peters RD, Henkelman RM, Piewes DB, Bronskill MJ, Kucharczyk W. (1998) MRI monitoring of interstitial microwave-induced heating and thermal lesions in rabbit brain in vivo. JMRI. 8:128-135.

Morris DL, McArdle CS, Onik GM. (1996) Hepatic metastases: diagnosis and management. 1:1-179.

Mumtaz H, Hall-Craggs MA, Wotherspoon A, Paley M, Buonaccorsi G, Amin Z, Wilkinson I, Kissin MW, Davidson TI, Taylor I, Bown SG. (1996) Laser therapy for breast cancer: MR imaging and histopathologic correlation. Radiology. 200:651-658.

Murrer LH, Marijnissen JP, Star WM. (1997) Improvements in the design of linear diffusers for photodynamic therapy. Phys. Med Biol. 42:1461-1464.

Nagasue N, Yukaya H, Ogawa Y, Hirose S, Okita M. (1985) Segmental and subsegmental resections of the cirrhotic liver under hepatic inflow and outflow occlusion. Br. J. Surg. 72:565-568.

Neumann RA, Knobler R, Leonhartsberger H, Bohler-Sommeregger K, Gebhart W. (1991) Histochemical evaluation of the coagulation depth after argon laser impact on a port-wine stain. Lasers Surg Med. 11:606615 .

Nolsoe CP, Torp-Pedersen S, Horn T, Larson LG, Lorentzen T, Holm HH. (1989) Interstitial laser ablation of tumors- follow up biopsies implies a risk of misinterpretation. Lasers Surg Med.21

Nolsoe CP, Torp-Pedersen S, Burcharth F, Horn T, Pedersen S, Christensen NE, Olldag ES, Andersen PH, Karstrup S, Lorentzen T. (1993) Interstitial hyperthermia of colorectal liver metastases with a US-guided NdYAG laser with a diffuser tip: a pilot clinical study. Radiology. 187:333-337.

Nordlinger B, Guiguet M, Vaillant JC, Balladur P, Boudjema K, Bachellier P, Jaeck D. (1996) Surgical resection of colorectal carcinoma metastases to the liver. A prognostic scoring system to improve case selection, based on 1568 patients. Cancer. 77:1254-1262.

Orth K, Russ D, Hibst R, Mattfeldt T, Steiner R, Beger HG. (1997) Laser coagulation zones induced with the Nd-YAG laser in the liver. Lasers Med Sci. 12:137-143. 
Parker DL, Smith V, Sheldon P, Crooks LE, Fussel L. (1983) Temperature distribution measurements in two-dimensional NMR imaging. Med Phys. 10:321-325.

Patterson EJ, Scudamore CH, Owen DA, Nagy AG, Buczkowski AK. (1998) Radiofrequency ablation of porcine liver in vivo. Effects of bloodflow and treatment time on lesion size. Anm. Surg. 227:559-565.

Peters RB, Hinks RS, Henkelman RM. (1996) Ex vivo tissue-type invariability in proton-resonance frequency shift MR thermometry. In Proceedings of the International Society for Magnetic Resonance in Medicine. Fourth scientific meeting, p 1955 Society of Magnetic Resonance in Medicine: Berkeley, Ca.

Peterson HI. (1979) Tumor Blood Circulation: angiogenesis, vascular morphology and blood flow of experimental and human tumours.1-227.

Prapavat V, Roggan A, Walter J, Beuthan J, Klingbell U, Muller G. (1996) In vitro studies and computer simulations to assess the use of a diode laser $(850 \mathrm{~nm})$ for laser-induced thermotherapy (LITT). Lasers Surg Med. 18:22-33.

Prudhomme M, Rouy S, Tang G, Godlewski G, Delacretaz G, Schonenberger K, Rink K. (1996) Partial response to laser-induced thermotherapy: incidence of biliary structures. In Laser Applications in Medicine and Dentistry (Proc SPIE 2922), Altshuler GB et al. (eds). Pp 362-365. SPIE: Bellingham.

Prudhomme M, Rouy S, Tang S, Landgrebe J, Delacretaz G, Godlewski G. (1999) Biliary structures lead to tumour recurrences after laser-induced interstitial thermotherapy. Lasers Surg Mfed. 24:269-275.

Pushek T, Farahani K, Saxton RE, Soudant J, Lufkin R, Paiva M, Jongewaard N, Castro DJ. (1995) Dynamic MRI-guided interstitial laser therapy: a new technique for minimally invasive surgery. Laryngoscope. 105:1245-1252.

Ravikumar TS, Kane R, Jenkins JR, Clouse M, Stcele G, Jr. (1991a) A 5-year study of cryosurgery in the treatment of liver tumors. Arch. Surg. 126:1520-1524.

Ravikumar TS, Steele G, Jr., Kane R, King V. (199lb) Experimental and clinical observations on hepatic cryosurgery for colorectal metastases. Cancer Res. 51:6323-6327.

Ravikumar TS, Buenaventura S, Salem RR, D'Andrea B. (1994) Intraoperative ultrasonography of liver: detection of occult liver tumors and treatment by cryosurgery. Cancer Detect. Prev. 18:131-138.

Ravikumar TS. (1996) The role of cryotherapy in the management of patients with liver tumors. Advances in Surgery:281-291.

Rees M, Plant G, Bygrave S. (1997) Late results justify resection for multiple hepatic metastases from colorectal cancer. BrJ Surg. 84:1136-1140.

Ritchie KP, Keller BM, Syed KM, Lepock JR. (1994) Hyperthermia (heat-shock) induced protein denaturation in liver, muscle and lens tissue as determined by differential scanning calorimetry. Int. J. Hyperthermia. 10:605-618.

Ritz JP, Roggan A, Germer CT, Albrecht D, Buhr HJ. (1997) Determination of optical parameters of human liver tissue and fiver metastases for irradiation planning by laser-induced thermotherapy of liver metastases. Langenbecks. Arch. Chir: Suppl. Kongressbd. 114:335-338.

Roberts HR, Paley M, Hall-Craggs MA, Lees WR, Friedman EP, ClemenceM., Buonacorssi G, Bown SG. (1994) Dynamic magnetic resonance control of interstitial laser photocoagulation therapy of colorectal hepatic metastases [letter]. Lancet. 343:1221

Roggan A and Muller G. (1995) Dosimetry and computer based irradiation planning for laser- induced interstitial thermotherapy (LITT). In Laser-induced Interstitial Thermotherapy, Roggan A and Muller G(eds.) pp 114-157. SPIE: Bellingham.

Ross WB, Horton M, Bertolino P, Morris DL. (1995) Cryotherapy of liver tumours: a practical guide. $H P B$. Surg. 8:167-173.

Rossi S, Di Stasi M, Buscarini E, Quaretti P, Garbagnati F, Squassante L, Paties CT, Silverman DE, Buscarini L. (1996) Percutaneous RF interstitial thermal ablation in the treatment of hepatic cancer. $A J R$. Am. J. Roentgenol. 167:759-768.

Saint-Jalmes H. (1995) Precision in temperature measurement via T1 or diffusion imaging. In Proceedings of the International Society for Magnetic Resonance in Medicine. Third scientific meeting, p 1072 Society of Magnetic Resonance in Medicine: Berkeley, Ca. 
Samulski TV, Macfall J, Zhang Y, Grant W, Charles C. (1992) Non-invasive thermometry using magnetic resonance diffusion imaging: potential for application in hyperthermic oncology. Int. J. Hyperthermia. 8:819-829.

Scheele J, Stang R, Altendorf-Hofmann A, Paul M. (1995) Resection of colorectal liver metastases. World $J$ Surg. 19:59-71.

Schober R, Bettag M, Sabel M, Ulrich F, Hessel S. (1993) Fine structure of zonal changes in experimental Nd:YAG laser-induced interstitial hyperthermia. Lasers Surg Med. 13:234-241.

Schroder T, Castren-Persons M, Lehtinen A, Taavitsainen M. (1994) Percutaneous interstitial laser hyperthermia in clinical use. Ann. Chir. Gynaecol. 83:286-290.

Schulze CP, Kahn T, Harth T, Schwarzmaier HJ, Schober R. (1998) Correlation of neuropathologic findings and phase-based MRI temperature maps in experimental laser-induced interstitial thermotherapy. JMRI. 8:115-120.

Schwabe B, Kahn T, Harth T, Utrich F, Schwarzmaier HJ. (1997) Laser-induced thermal lesions in the human brain: short- and long-term appearance on MRI. J. Comput. Assist. Tomogr: 21:818-825.

Scudamore CH, Patterson EJ, Shapiro AM, Buczkowski AK. (1997) Liver tumor ablation techniques (review). J. Invest. Surg. 10:157-164.

Semelka RC and Ascher SM. (1993) MR imaging of the pancreas. Radiology. 188:593-602.

Shah K and Gerber M. (1990) Development of intrahepatic bile ducts in humans. Arch Pathol Lab Med. 114:597-600.

Sibille A, Prat F, Chapelon JY, Abou el Fadil F, Henry L, Theillere Y, Ponchon T, Cathignol D. (1993) Extracorporeal ablation of liver tissue by high-intensity focused ultrasound (Review). Oncology. 50:375-379. Sinha S, Oshiro T, Sinha U, Lufkin R. (1997) Phase imaging on a .2-T MR scanner: application to temperature monitoring during ablation procedures. J. Magn. Reson. Imaging. 7:918-928.

Slooter GD, Marquet RL, Jeekel J, IJzermans JNM. (1995) Tumour growth stimulation after partial hepatectomy can be reduced by treatment with tumour necrosis factor alpha. Br. J. Surg, 82:129-132.

Solbiati L, Ierace T, Goldberg SN, Sironi S, Livraghi T, Fiocca R, Servadio G, Rizzatto G, Mueller PR, Del Maschio A, Gazelle GS. (1997) Percutaneous US-guided radio-frequency tissue ablation of liver metastases: treatment and follow-up in 16 patients [see comments]. Radiology. 202:195-203.

Stangl R, Altendorf-Hofmann A, Chanley RM, Scheele J. (1994) Factors influencing the natural history of colorectal liver metastases. Lancet. 343:1405-1410.

Steele G, Jr., Bleday R, Mayer RJ, Lindblad A, Petrelli N, Weaver D. (1991) A prospective evaluation of hepatic resection for colorectal carcinoma metastases to the liver: Gastrointestinal Tumor Study Group Protocol 6584. J Clin Oncol. 9:1105-1112.

Steele G, Jr. (1996) Hepatic metastasis resection: results. In Hepatic metastases: diagnosis and management, Morris DL, McArdle CS, anci Onik GM(eds.) pp 57-66. Butterworth-Heineman: Oxford.

Steger AC, Lees WR, Shorvon P, Walmsley K, Bown SG. (1992a) Multiple-fibre low-power interstitial laser hyperthermia: studies in the normal liver. Br. J. Surg. 79:139-145.

Steger AC, Shorvon P, Walmsley K, Chisholm R, Bown SG, Lees WR. (1992b) Ultrasound features of low power interstitial laser hyperthermia. Clin. Radiol. 46:88-93.

Steiner P, Botnar R, Goldberg SN, Gazelle GS, Debatin JF. (1997) Monitoring of Radio Frequency tissue ablation in an interventional magnetic resonance enveronment. Invest. Radiol. 32:671-678.

Stollberger R, Ascher PW, Huber D, Renllart W, Radner H, Ebner F. (1998) Temperature monitoring of interstitial thermal tissue coagulation using MR phase inages. JMRI. 8:188-196.

Strohmeyer T, Haugeberg G, Lierse W. (1987) Angioarchitecture and blood supply of micro- and macrometastases in human livers. An anatomic-pathological investigation using injection techniques. $J$ Hepatol. 4:181-189.

Surh CD and Sprent J. (1994) T-cell apoptosis detected in situ during positive and negative selection in the thymus. Nature. 372:100-103.

Svaasand LO, Boerslid T, Oeveraassen M. (1985) Thermal and optical properties of living tissue: application to laser-induced hyperthermia. Lasers Surg Med. 5:589-602.

Svaasand LO, Gomer CJ, Morinelli E. (1990) On the physical rationale of laser induced hyperthermia. Lasers Med Sci. 5:121-127. 
Sykes MK. (1989) High frequency ventilation (Review). Br. J. Anaesth. 62:475-477.

Taber $\mathrm{KH}$ and Hayman AL. (1992) Temperature monitoring during MR imaging: comparison of fluoroptic and standard thermistors. J. Magn. Reson. Imaging. 2:99-101.

Taourel P, Blanc P, Dauzat M, Chabre m, Pradel J, Gallix B, Larrey D, Bruel J. (1998) Doppler study of mesenteric, hepatic and portal circulation in alcoholic cirrhosis: relationship between quantitative doppler measurements and the severity of portal hypertension and hepatic failure. Hepatology. 28:932-936.

Thomsen S. (1991) Pathologic analysis of photothermal and photomechanical effects of laser-tissue interaction. Photochem. Photobiol. 53:825-835.

Thomsen S. (1995) Identification of lethal thermal injury at the time of photothermal treatment. In Laserinduced interstitial thermotherapy, Roggan A and Muller G(eds.) pp 459-467. SPIE: Bellingham.

Tracz RA, Wyman DR, Little PB, Towner RA, Stewart WA, Schatz SW, Pennock PW, Wilson BC. (1992) Magnetic resonance imaging of interstitial laser photocoagulation in brain. Lasers Surg Med. 12:165-173.

Tracz RA, Wyman DR, Little PB, Towner RA, Stewart WA, Schatz SW, Wilson BC, Pennock PW, Janzen EG. (1993) Comparison of magnetic resonance images and the histopathological findings of lesions induced by interstitial laser photocoagulation in the brain. Lasers Surg Med. 13:45-54.

Tranberg KG, Moller PH, Hannesson P, Stenram U. (1996) Interstitial laser treatment of malignant tumours: initial experience. Eur J Surg Oncol. 22:47-54.

Trondsen E, Rosseland AR, Bakka A, Ruud TE, Martinsen T, Clausen OPF, Bergan A, Aasen AO. (1998) An experimental study on effects of monopolar diathermy on the bile ducts. Eur. J. Surg. 164:297-303.

Vahrmeijer AL, van Dierendonck JH, van de Velde CJ. (1995) Treatment of colorectal cancer metastases confined to the liver. Eur J Cancer. 31A:1238-1242.

van Hillegersberg R, de Witte MT, Kort WJ, Terpstra OT. (1993a) Water-jet-cooled Nd:YAG laser coagulation of experimental liver metastases: correlation between ultrasonography and histology. Lasers Surg Med. 13:332-343.

van Hillegersberg R, Pickering JW, Aalders M, Beek JF. (1993b) Optical properties of rat liver and tumor at $633 \mathrm{~nm}$ and $1064 \mathrm{~nm}$ : photofrin enhances scattering. Lasers Surg Med. 13:31-39.

van Hillegersberg R, van Staveren HJ, Kort WJ, Zondervan PE, Terpstra OT. (1994) Interstitial Nd:YAG laser coagulation with a cylindrical diffusing fiber tip in experimental liver metastases. Lasers Surg Med. 14:124-138.

van Hillegersberg R, van Staveren HJ, Roggan A, Muller G, JJzermans JNM. (1995) Interstitial laser coagulation as a treatment for breast cancer [letter]. $\mathrm{Br} J$ Surg. 82(6):856

van Hillegersberg R. (1997) Fundamentals of laser surgery. Eur: J. Surg. 163:3-12.

Vehmas T, Taari K, Kivisaari L, Laurila P, Taavitsainen M. (1993) Intra- and peri-hepatic effects of ethanol injections in the pig liver. Scand. J. Gastroenterol. 28:791-794.

Venables VV and Ripley BD. (1994) Statistics with S-Plus.154-164.

Venesmaa P and Ylikorkala O. (1991) Subrenal capsule assay in selection of chemotherapy after operation for recurrent ovarian cancer. Br. J. Cancer, 63:84-86.

Verheul M. (1998) Aggregation and gelation of whey proteins. Thesis, University of Twente.

Vitkin IA, Moriarty JA, Peters RD, Kolios MC, Gladnan AS, Chen JC, Hinks RS, Hunt JW, Wilson BC, Easty AC, Bronskill MJ, Kucharczyk W, Sherar MD, Henkelman RM. (1997) Magnetic resonance imaging of temperature changes during interstitial microwave heating: a phantom study. Med Phys. 24:269-277.

Vog1 TJ, Muller PK, Hammerstingl R, Weinhold N, Mack MG, Philipp C, Beuthan J, Pegios W, Riess H. (1995) Malignant liver tumors treated with MR imaging-guided laser-induced thermotherapy: technique and prospective results. Radiology: 196(1):257-265.

Vogl TJ, Weinhold N, Muller P, Phillip C, Roggan A, Mack MG, Balzer JO, Eichstadt H, Blumhardt G, Lobeck H, Felix R. (1996) Early clinical experiences with MR-guided laser-induced thermotherapy (LITT) of liver metastases in preoperative care. Rofo. Fortschr. Geb. Rontgenstr. Netuen. Bildgeb. Verfahr. 164:413421.

Vogl TJ, Mack MG, Straub R, Roggan A, Felix R. (1997) Percutaneous MRI-guided laser-induced thermotherapy for hepatic metastases for colorectal cancer (letter). Lancet. 350:29 
Vogl TJ, Mack MG, Roggan A, Straub R, Eichler KC, Muller PK, Knappe V, Felix R. (1998) Internally cooled power laser for MR-guided interstitial laser-induced thermotherapy of liver lesions: initial clinical results. Radiology. 209:381-385.

Waiser EM, McNees SW, DeLa Pena O, Crow IVN, ., Morgan RA, Soloway R, Broughan T. (1998) Portal venous thrombosis: percutaneous therapy and outcome. JVIR. 9:119-127.

Wanebo HJ, Chu QD, Vezeridis MP, Soderberg C. (1996) Patient selection for hepatic resection of colorectal metastases. Arch Surg. 131:322-329.

Weaver ML, Atkinson D, Zemel R. (1995) Hepatic cryosurgery in treating colorectal metastases. Cancer. 76,2:210-214.

Weber S, Lee FT, Chinn DO, Warner T, Chosy SG, Mahvi D. (1997) Perivascular and intralesional tissue necrosis after hepatic cryoablation: Results in a porcine model. Surgery: 122:742-747.

Welch AJ, Motamedi M, Rastegar S, LeCarpentier GL, Jansen D. (1991) Laser thermal ablation. Photochem. Photobiol. 53:815-823.

Whelan WM, Wyman DR, Wilson BC. (1995) Investigations of large vessel cooling during interstitial laser heating. Med. Phys. 22:105-115.

Wilson DL, Carillo A, Zheng L, Genc A, Duerk JL, Lewin JS. (1998) Evaluation of 3D image registration as applied to MR-guided thermal treatment of liver cancer. JMRI. 8:77-84.

Wyman d, Wilson B, Adams K. (1994) Dependence of laser photocoagulation on interstitial delivery parameters. Lasers Surg Med. 14:59-64.

Wyman DR, Whelan WM, Wilson BC. (1992) Interstitial laser photocoagulation: Nd:YAG $1064 \mathrm{~nm}$ optical fiber source compared to point heat source. Lasers Surg Med. 12:659-664.

Wyman DR. (1993) Selecting source locations in multifiber interstitial laser photocoagulation. Lasers Surg Med. 13:656-663.

Xu Y and Qian R. (1995) Analysis of thermal injury process based on enzyme deactivation mechanisms. Jounal of Biomechanical Engineering. 117:462-465.

Yamamoto J, Shimada K, Kosuge T, Yamasaki S, Sakamoto M, Fukuda H. (1999) Factors influencing survival of patients undergoing hepatectomy for colorectal metastases. Br J Surg. 86:332-337.

Yang R, Sanghvi NT, Rescorla FJ, Kopecky KK, Grosfeld JL. (1993) Liver cancer ablation with extracorporeal high-intensity focused ultrasound (Review). Eur: Urol. 23 Suppl 1:17-22.

Young IR, Hand JW, Oatridge A, Prior MV. (1994a) Modeling and observation of temperature changes in vivo using MRI. Magn Reson. Med. 32:358-369.

Young IR, Hand JW, Oatridge A, Prior MV, Forse GR. (1994b) Further observations on the measurement of tissue T1 to monitor temperature in vivo by MRI. Magn Reson. Med. 31:342-345. 


\section{Summary}

\section{Part I General introduction}

\section{Chapters 1 \& 2: Overview of $\mathrm{LC}$ and aims of the thesis}

Surgical resection is the only curative treatment currently available for patients with hepatic metastases of colorectal carcinoma and hepatocellular carcinoma. However, only 10-20\% of patients are eligible for surgery and the operative morbidity and mortality rates are relatively high owing to associated normal tissue damage. These limitations may be overcome by Interstitial laser coagulation (ILC), a technique of localised thermal tissue destruction. With regard to tumour response the promising results, obtained in animal experiments, could not be confirmed in clinical studies. In tumours larger than approximately $2.5 \mathrm{~cm}$ in diameter the result is variable and tumours are often incompletely destroyed, necessitating repeated treatment. In summary, two factors contribute: 1) The small volume of tissue that can be coagulated in a single treatment session and 2) The lack of reliable monitoring of the laser-induced effects during ILC. Experimental studies to improve both aspects were prepared.

\section{Part II Increasing lesion size}

\section{Chapter 3: Diffusing-tip fibre versus bare-tip fibre}

A heat resistant cylindrical light diffusing tip of $2 \mathrm{~cm}$ length was developed for this application. We performed an in vitro study to compare this diffusing-tip with a bare-tip fibre. The fibre ends were positioned between two porcine liver slabs $\left(37^{\circ} \mathrm{C}\right)$ and $\mathrm{Nd}$ :YAG laser light $(1064 \mathrm{~nm})$ was guided through either fibre with an output of 3-9 W and exposure times of 6-18 minutes. The lesions produced by the cylindrical diffuser tip were significantly larger and more predictable. With the diffuser tip, lesions up to $36 / 23 \mathrm{~mm}$ (length/diameter) could be produced at $7 \mathrm{~W}$ and 9 min without any central charring. The maximum size of lesions produced with the bare-tip fibre was $32 / 20 \mathrm{~mm}$ at $6 \mathrm{~W}$ for 9 min with massive charring. 


\section{Chapter 4: Simultaneous multiple fibre application}

This could increase the volume of coagulation produced with ILC. For that purpose an optical beamsplitter was constructed, dividing high power Nd:YAG light into 4 equal beams (maximal variation $9.5 \%$, transmission always $>85 \%$ ). To take full advantage of the presumed synergistic thermal effect between the interstitially applied fibres, the optimal combination of laser power and distance between the fibres was assessed. Per fibre 4-7 W was guided. Fibres were positioned in a square fashion in ex vivo porcine liver. By adjusting exposure time, energy per fibre was constant at 1800 J. Distance between the fibres was varied between $1-4 \mathrm{~cm}$. After laser application, dimensions of the lesions were measured. The optimal mutual fibre distance was $2 \mathrm{~cm}(\mathrm{P}<0.0001)$ at all power levels. This resulted in lesions with a mean (SD) volume of $44.5(2.1) \mathrm{cm}^{3}$ and a largest diameter of $5.1(0.4) \mathrm{cm}$ at $7 \mathrm{~W}$ per fibre. Smaller distances between the fibres resulted in smaller lesions with central carbonisation, whereas larger distances resulted in 4 separate zones of coagulation.

\section{Chapter 5: Eliminating portal flow}

Perfusion by the vascular system can transfer heat from the treatment site, thereby reducing the volume of the destroyed tissue. The magnitude of this effect was investigated in a pig model $(n=10)$. Lesions were produced in the liver with 4 interstitial laser fibres ( $5 \mathrm{~W}$ per fibre during 6 minutes): 1) with flow through the liver $(n=9), 2)$ with portal vein clamped $(n=8)$ and 3 ) with both hepatic artery and portal vein clamped $(n=6)$. The pigs were killed and the liver was dissected; lesions were assessed macroscopically and microscopically. Lesion volume was significantly larger when portal flow was eliminated, $30.6 \mathrm{~cm}^{3}$ compared to $6.5 \mathrm{~cm}^{3}(P<0.001)$. Hepatic inflow substantially reduces the lesion size produced by interstitial laser coagulation. Occlusion of the arterial flow with the portal flow did not result in an additional increase of the volume of coagulation.

\section{Part III Laser-tissue interaction and monitoring}

\section{Chapter 6: Perivascular effects of ILC with inflow occlusion}

The effects of portal inflow occlusion during ILC on adjoining major intrahepatic structures are unknown. Therefore the purpose was to assess the effects of ILC with portal inflow occlusion on the central portion of the liver as compared to the peripheral portions. In 16 healthy pigs, ILC was performed with portal inflow occlusion. Each animal received a single laser application with 
Nd:YAG light guided simultaneously through 4 interstitial fibres with $5 \mathrm{~W}$ per fibre during 6 minutes. Lesions were induced either central $(n=8)$ or peripheral $(n=8)$ in the liver. Follow-up was for 1,2 or 3 months by an evaluation of liver functions and macroscopic and microscopic assessment of coagulated lesions and surrounding parenchyma. There was no treatment related morbidity or mortality. No significant differences between the 2 groups (liver functions $[P \geq 0.12]$ and weight $[P=0.73]$ ) were found. At microscopic evaluation the coagulation necrosis was encased in a fibrous sheet of which the contents were resorbed in time. At every moment of evaluation, coagulated volumes in the central group were smaller than in the peripheral lesions $(P=0.03)$. Large vessels neighbouring to lesions were always intact and no indications for portal hypertension or thrombosis in hepatic veins were found.

\section{Chapter 7: Lethality of $\mathrm{LC}$}

Tissue whitening is associated with global themal denaturation but is not a good indicator of the exact outer boundary of the lethal tissue effect. To this end two enzymatic methods were investigated. ILC was performed in human hepatic metastases of colorectal carcinoma; directly after surgical resection $(n=5)$ or prior to surgical resection $(n=5)$. Laser parameters were adapted to tumour diameter. Metabolic activity was measured with the mitochondrial NADH-diaphorase activity and integrity of the DNA was assessed by staining for blund DNA fragments (TUNEL). $H \& E$ sections were used to define the corresponding morphological areas. In 7 out 10 tumours the area of macroscopic coagulation encircled the tumour in all three axes. The volume of coagulation was on average 1.8 larger than the tumour volume (range: 0.34 - 4.2). Macroscopic coagulation corresponded to absent metabolism and disintegrated DNA. No islands of vital cells were found within the area of coagulation. Furthermore the volumes of coagulation produced in tumour were comparable to the dimensions in normal porcine liver with the same laser parameters.

\section{Chapter 8: Critical isotherm for tissue coagulation}

Although it is generally assumed that in solid tissue and tumour, warmed at $60^{\circ} \mathrm{C}$ for several minutes, complete protein denaturation occurs, there are no supporting experimental data. Samples $\left(8 \mathrm{~mm}^{3}\right)$ of solid rat tumour (a CC-531 syngenic to the WAG/Rij rat strain), were warmed in tubes for 4 different temperatures $\left(40^{\circ} \mathrm{C}, 50^{\circ} \mathrm{C}, 60^{\circ} \mathrm{C}\right.$ or $\left.80^{\circ} \mathrm{C}\right)$ and 4 different exposure times $(3,6,12,24$ min). Combinations were replicated in 5 fold. Cell viability was assessed with 3 methods: Trypan blue exclusion test in collagenase/dispase dissociated samples, NADH activity in snap frozen 
samples and outgrowth for 2 weeks under the renal capsule of WAG/Rij rats. The three methods revealed that viability was not affected with heating at $40^{\circ}$ and $50^{\circ} \mathrm{C}$ except for 24 minutes at $50^{\circ} \mathrm{C}$. At higher temperatures cell death occurred at all exposure times.

\section{Chapter 9: MR-thermometry}

Several magnetic resonance techniques are suitable for temperature measurements. One of those: MR phase-shift mapping is a subtraction technique and therefore prone to the influence of movement of the abdomen and liver. The accuracy of MR-phase mapping for temperature monitoring was therefore investigated in two ex vivo models with simulated respiration and in pigs. The MR temperatures were calibrated to interstitially measured temperature. Gradual cooling of a homogenous medium (gel) was monitored for 4 starting temperatures (room temperature, $40^{\circ} \mathrm{C}$, $50^{\circ} \mathrm{C}$ and $60^{\circ} \mathrm{C}$ ) during 30 minutes. Temperature increases were measured during interstitial laser coagulation in ex vivo porcine liver with $\mathrm{Nd}$ :YAG for 6 minutes with $5 \mathrm{~W}$. Experiments were performed at rest and with simulated respiratory motion (both $n=5$ ). In liver, accuracy did not decrease with respiration simulation $(P=0.32)$, whereas a significant decline was found in the gel model $(P=0.002)$. In all experiments a small drift over time was observed between temperature determined with MR and thermoprobes. Correction for temperature independent phase-shift at a reference location did not enhance agreement. In pigs various factors resulted in massive artefacts.

\section{PART IV GENERAL DISCUSSION}

\section{Chapters 10 \& 11: Discussion and conclusions}

In conclusion it is demonstrated that complete coagulation can be achieved in volumes to $5.5 \mathrm{~cm}$ in diameter. In our view the results of the pre-clinical studies in this thesis justify clinical evaluation to the role of $\mathrm{LC}$ with portal inflow occlusion, multiple fibre- and water-cooled fibre- application in the treatment of patients with hepatic tumours. We hypothesise that these modifications result in a higher proportion of successful treatment in a single session for larger tumours. As a first step the safety, feasibility and efficacy of percutaneous $\Pi C$ with inflow occlusion wil be investigated in patients, ineligible for surgery. It is realised that the results in this stage will depend on the status of the online monitoring of the coagulation process. 


\section{Samenvatting voor de leek}

Voor een uitzaaiing van dikke- of endel- darm kanker naar de lever geldt, in tegenstelling tot de meeste andere uitgezaaide kwaadaardige ziekten dat verwijdering van de uitzaaiing kans biedt op genezing. Zo'n partiële leverresectie is ook de behandeling van keuze bij gezwellen uitgaande van de lever zelf. Helaas komt maar een gedeelte van deze groep patiënten in aammerking voor een gedeeltelijke lever resectie. En omdat, na cen verwijdering van alle tumoren te weinig lever over zou blijven en omdat, de algehele conditie van de patiënt zo'n grote operatie niet altijd toelaat. Er wordt wereldwijd onderzoek gedaan naar andere behandelingen voor deze patiëntengroep. De bestaande chemotherapie wordt verbeterd en verfijnd. En er is de ontwikkeling van nieuwe methoden die lokaal tumor destructie veroorzaken (en daarbij het omliggende weefsel sparen). Eén van die methoden is weefsel coagulatie door middel van laserlicht. Op het Laboratorium voor de Chirurgie wordt sinds 1988 onderzoek naar deze methode verricht. De werking lijkt op wat zich afspeelt bij het koken van een ei. Door verhitting van het weefsel ontstaat stolling van eiwitten. De hitte wordt gegenereerd door laserlicht. Dit wordt door een glasfiber, van 1 tot 2 millimeter in doorsnede, geleid tot in de tumor. Het licht dringt enige millimeters in het weefsel door en wordt geabsorbeerd. Er ontstaat een reactie waarbij hitte ontwikkeling optreedt. Ongeveer boven de $60^{\circ} \mathrm{C}$ ontstaat denaturatie van de eiwitten in de cellen en in het bindweefsel; hierbij ontstaat coagulatie, stolling, van het weefsel. Het effect treedt dus in het weefsel op (interstitieel), vandaar de naam interstitiële laser coagulatie (ILC).

Uit experimenten in proefdieren is gebleken dat de eiwitdenaturatie leidt tot volledige celddood in een duidelijk omschreven gebied. Ook de eerste resultaten in patiënten zijn veelbelovend. De behandeling is percutaan mogelijk. Daarbij wordt de glasfiber door de huid tot in de tumor geleid en hoeft de buik niet te worden geopend. De laserbehandeling was op die manier veilig en goed te verdragen door de patiënten. Het effect op de tumor effect blijkt afhankelijk te zijn van de grootte van de tumor. De grens waarboven de kans op succes kleiner wordt ligt op ongeveer $2.5 \mathrm{~cm}$. Hier dragen 2 factoren aan bij: 1) de hoeveelheid weefsel wat in één laser sessie gecoaguleerd kan worden is te gering en 2) ontbreekt het nog aan een techniek om al tijdens de behandeling, nauwkeurig de grootte van het laser effect vast te stellen. Dit kan nu alleen nog achteraf. 
In hoofdstuk 1 is dieper ingegaan op de geschiedenis, achtergrond en huidige stand van zaken van ILC voor levertumoren. In hoofdstuk 2 zijn hier conclusies aan verbonden en aanknopingspunten voor de verbetering van ILC geformuleerd.

\section{Vergroten van de lesie grootte}

In deel II zijn de mogelijkheden onderzocht om het coagulatie effect te vergroten. Hoofdstuk 3 richtte zich op het uiteinde van de glasfiber. Een nieuw ontwikkelde glasfiber, waarbij het laserlicht homogeen over de tip van 2 centimeter werd uitgezonden, werd vergeleken met de traditionele fiber met een tip waarbij het licht alleen vanaf de punt wordt uitgezonden. Met beide fibers werden tussen 2 plakken varkenslever coagulatie lesies aangebracht. Lesies met de nieuwe verstrooiende fiber waren groter en bovendien bleek de spreiding kleiner te zijn bij ILC met dezelfde instellingen. Zeven Watt gedurende 9 minuten leidde met de verstrooiende fiber-tip tot coagulatie in een gebied van 36 bij $23 \mathrm{~mm}$.

In hoofdstuk 4 werd onderzocht of belichting van 4 fibers gelijktijdig, een extra effect zou opleveren Dit was voorspeld in computer modellen. Een praktisch bewijs was uitgebleven door gebrek aan apparatuur om een laserbundel te splitsen in 4 evemredige bundels zonder groot energie verlies. Wij hebben een dergelijke bundelsplitser onderzocht en de optimale onderlinge afstand tussen de fibers bepaald. Deze afstand bleek 2 centimeter te zijn. Met een grotere onderlinge afstand $(4 \mathrm{~cm})$ trad geen vervloeiing van de 4 coagulatie gebieden rondom de fibers op; bij een afstand van $1 \mathrm{~cm}$ trad in het centrum van de 4 fibers een zodanige accumulatie van hitte op dat verkoling het gevolg was. De diameter van de coagulatie, aangebracht met $2 \mathrm{~cm}$ tussen de fibers, was gemiddeld $5.1 \mathrm{~cm}$.

In deze hoofdstukken 3 en 4 werden de studies in varkenslever, buiten het lichaam, uitgevoerd. De volgende stap, onderzoek in proefdieren, werd in hoofdstuk 5 gezet. Vanwege de grootte van de laser effecten werd gekozen voor varkens. Theoretisch was het mogelijk dat bloeddoorstroming, een koelend effect zou genereren en daardoor het laser effect verkleinen. Hiertoe zijn in 10 varkens lesies aangebracht met 1) normale doorbloeding, 2) de poortader afgesloten of 3) met én de poortader én de slagader naar de lever afgesloten. Afsluiting van de doorstroming van de poortader bleek tot een vervijfvoudiging van de grootte van het laser effect te leiden. Tevens de slagader af sluiten had geen toegevoegde waarde. Gemiddeld waren de lesies $4.5 \mathrm{~cm}$ in diameter. 


\section{Laser-weefsel interactie en beeldvorming}

De bevinding dat de doorbloeding tijdens de laserbehandeling moest worden stil gelegd, bracht een dilemma met zich mee. Eerder was het gesuggereerd dat de grote vaten en galwegen door die doorstroming juist beschermd zouden worden tegen de schade door de hitte. In 2 groepen van 8 varkens hebben wij de laserhandeling uitgevoerd, net als in hoofdstuk 5 , met onderbreking van de portale flow. ILC in de rand van de lever werd vergeleken met ILC centraal in de lever. Er werden geen complicaties gezien. Het bleek dus dat de grote vaten en galwegen goed bestand zijn tegen de gegenereerde hitte. Wel was de grootte van het coagulatie effect, groter in perifere lesies. Dit onderstreepte het belang van betrouwbare real-time registratie van de laser effecten.

Tot voor kort was het niet mogelijk om direct na de laserbehandeling vast te stellen of een cel definitief was beschadigd. In proefdiermodellen kon dat alleen door in de tijd de weefseleffecten te vervolgen. In patiënten zou dit alleen met herhaalde biopten mogelijk zijn met het daaraan verbonden ongemak en gebrek aan overzicht over de gehele tumor. In hoofdstuk 7 werd in de resectie preparaten van patiënten met levertumoren ILC toegepast. In weefsel coupes werd met twee nieuwe methoden zowel de energie huishouding als het genetisch materiaal aangekleurd. Het bleek dat in de gebieden waarin macroscopisch volledige coagulatie was vastgesteld, de cellen inderdaad definitief beschadigd waren. In drie tumoren waarin op het oog onvolledige coagulatie had plaatsgevonden waren nog vitale tumorcellen achtergebleven.

De hypothese dat verwarming tot boven $60^{\circ} \mathrm{C}$ leidt tot voldoende eiwtdenaturatie en weefselcoagulatie is ontstaan na experimenten in eiwitoplossingen. Voor solide fumor weefsel was dit nog niet bekend. In hoofdstuk 7 is beschreven hoe wij fragmenten van een rattentumor op 4 verschillende temperaturen en 4 verschillende tijdsduren verwarmen in een waterbad. $\mathrm{Na}$ verwarming werd de vitaliteit met 3 verschillende methoden onderzocht. Het bleek dat verhitting tussen de $50^{\circ}$ en $60^{\circ} \mathrm{C}$ gedurende 3 minuten voldoende eiwitschade geeft om tumordood te bewerkstelligen. Op hogere temperaturen of gedurende langere tijd verhitten was dus niet nodig.

De kennis, vergaard in de hoofdstukken 6-8, was een aanloop tot de studie beschreven in hoofdstuk 9. Magnetische kern-spin resonantie werd gebruikt om de temperatuurtoename van het weefsel tijdens ILC te meten. Hiervoor lenen zich verschillende MRI technieken. De door onze gebruikte chemical shift imaging lijkt een goed compromis tussen de nauwkeurigheid van de bepaling en de tijd benodigd voor de scan. Het doel van de studie was tweeledig. Enerzijds het vaststellen van de nauwkeurigheid en op de tweede plaats de invloed van de ademhaling op de nauwkeurigheid bepalen. Tijdens ILC in lever had de gesimuleerde ademhaling geen effect. In een gel-monster 
waarin de afkoeling werd gemeten was er wel een afname van de nauwkeurigheid. Gedurende de experimenten nam de nauwkeurigheid waarmee de temperatuur bepaald kon worden af. Niettemin kon na 6 minuten laserbehandeling de temperatuur tot op $\pm 3.5^{\circ} \mathrm{C}$ bepaald worden. De eerste experimenten in varkens gaven bemoedigende resultaten. Wel werd het duidelijk dat een aantal aanpassingen in de techniek en software onontbeerlijk zij om in vivo dezelfde nauwkeurigheid te behalen als ex vivo. In deze experimenten werd tevens een nieuwe laser catheter beproefd die gedurende de laserbehandeling inwendig gekoeld werd. Hiermee kon langer belicht worden zonder kans op verkoling. Zones van coagulatie schade van gemiddeld $5.5 \mathrm{~cm}$ in doorsnede waren mogelijk.

Samenvattend kan gesteld worden dat volledige coagulatie aangebracht kan worden in een gebied met een diameter van $5.5 \mathrm{~cm}$. Hierin treedt histologisch bewezen volledige celdood op. Deze resultaten rechtvaardigen studies in patiënten naar ILC met vasculaire inflow occlusie, multiple fibers en water-gekoelde fibers. De hypothese is dat deze modificaties op de bestaande techniek, resulteren in een hoger percentage geslaagde laserbehandelingen in 1 sessie. Mogelijk zijn hierdoor minder herhaalde laserbehandelingen nodig. De eerste stap is het bepalen van de complicaties en haalbaarheid in een kleine groep patiënten met levertumoren, die geen kandidaat zijn voor een partiële leverresectie. De resultaten in deze periode zullen deels bepaald worden door de nauwkeurigheid van het afbeeldend onderzoek tijdens de laserbehandeling. 


\section{Contributing Authors}

R. van Hillegersberg MD PhD. Department of Surgery, Erasmus University and University Hospital Rotterdam Dijkzigt. At present: Department of Surgery, Ikazia Hospital Rotterdam.

Prof. J.S. Laméris MD PhD. Department of Radiology, Erasmus University and University Hospital Rotterdam Dijkzigt. At present: Department of Radiology, Academic Medical Centre, Amsterdam.

N.A.A Matheijssen. Department of Radiology, Erasmus University and University Hospital Rotterdam Dijkzigt.

P.H. Mulder PhD. Department of Epidemiology and Biostatistics. Erasmus University Rotterdam.

E.L. Sinofsky PhD. CardioFocus, West Yarmouth, Ma, United States of America.

J. Stoker MD PhD. Department of Radiology, Erasmus University and University Hospital Rotterdam Dijkzigt. At present: Department of Radiology, Academic Medical Centre, Amsterdam.

J.J. van Vaals PhD. Department of Clinical Science MR. Philips Medical Systems, Best.

J.N.M. IJzermans MD PhD. Department of Surgery, Erasmus University and University Hospital Rotterdam Dijkzigt,

P.E. Zondervan MD. Department of Pathology Erasmus University and University Hospital Rotterdam Dijkzigt. 



\section{Dankwoord}

Dat velen aan de totstandkoming van dit proefschrift hebben bijgedragen blijkt al uit de lijst van mede-auteurs aan de diverse hoofdstukken. De hulp, steun en vertrouwen van nog vele anderen is onontbeerlijk geweest. Enkelen wil ik in het bijzonder bedanken:

Maaike, als geen ander heb jij de wording van dit proefschrift meegemaakt. Lieve schat, met jouw aangeboren enthousiasme voor de wetenschap heb je ook mij aangestoken. Bovendien, gedeelde smart is halve smart: de komende maanden zijn de rollen omgedraaid en zal ik jou evenzeer bijstaan in de afronding van jouw proefschrift. Onovertroffen is jouw plezier in eigenlijk alle zaken die het leven veraangenamen, hierin laat ik mij graag meeslepen.

Mijn ouders en mijn zus Eva, jullie dank ik voor de stimulerende opvoeding, de continue interesse in het onderzoek en het meeleven met de voltooiing van dit proefschrift.

Prof. dr. J. Jeekel, al vroeg toonde u belangstelling voor de vorderingen van het onderzoek en was u bereid als promotor op te treden. Ik heb bewondering voor de snelheid warmee $\mathrm{u}$ een onderwerp analyseert, beheerst en vervolgens conclusies aan verbindt.

Dr. J.N.M. JJzermans en dr. R. van Hillegersberg, mijn co-promotores. Telkenmale realiseer ik me hoezeer ik heb geboft met de begeleiding door jullie beiden. Het enorme vertrouven en geduld waarmee jullie mij als student invoerden in het wetenschappelijk onderzoek was zeer stimulerend. Beste Richard, jouw gedrevenheid is een grote inspiratiebron. Beste Jan, de ontspannen wijze waarop jij hoofd en bijzaken weet te scheiden is onnavolgbaar. Jouv vertrouwen gaf mij menigmaal moed.

Prof. dr. G.P. Krestin, Prof. dr. G. Stoter en Prof. dr. O.T. Terpstra dank ik voor de bereidheid het manuscript te beoordelen en in de promotie commissie zitting te nemen. Aan Prof. dr. O.T. Terpstra ben ik extra dank verschuldigd, zonder Uw voornitziende blik was dit onderzoek nooit gestart in Rotterdam.

Dr. E.L. Sinofsky, dear Ed, your progress in fiber developments and contact with Richard led to the first studies, on which this thesis is based. I very much enjoyed the enthusiastic conversations with you and $I$ have respect for your combination of science and business.

Dr. Matheijssen, dr. J. Stoker en dr. J.J. van Vaals. MRI-thermometrie is geen gemakkelijk onderwerp! De ontwikkeling van deze pijler van het Rotterdamse laser-lever onderzoek was niet 
mogelijk geweest zonder jullie inzet. Ik dank jullie voor het engelengeduld waarop jullie mij de principes ervan hebben bijgebracht. Niels, geen tegenslag of we hebben hem meegemaakt. Herhaaldelijk verbaasde ik weer over jouw opgeruimdheid waarmee we vervolgens het volgende experiment voorbereidden.

De tweede pijler betrof de histologische analyse van de vele preparaten die de experimenten opleverden. Ik kon op de gekste momenten met een (reeds aangesneden) preparaat aan komen zetten. Dankzij Monica Seijbel, Josje van Loon, Cobi Peekstok, Frieda van de Ham was de verwerking nimmer een probleem. De heer P.E. Zondervan dank ik voor de kritische beschouwingen. Als geen ander weet $\mathfrak{u}$ bevindingen te relativeren.

Bij de medewerkers van het EDC waren de experimenten in goede handen. Hoe bizar de planning ook was, hoe laat de varkens ook van stal gehaald moesten worden, jullie stonden er altijd voor klaar. In het bijzonder dankzij Enno Collij, Henk Dronk, Rob Meijer, Janny de Kam en Roy Spruijt. Jullie onnavolgbare inventiviteit heeft menig experiment doen slagen.

De chirurgen Spillenaar Bilgen, van Eijck en Kazemier sprongen enthousiast in bij de operaties, mijn dank voor de vanzelfsprekende manier waarop jullie naar het laboratorium kwamen.

De dames van 10-Midden hebben heel wat logistieke problemen opgelost. Dankzij Scarlet van Belle was dia's maken nooit een probleem. Marry Nugteren, Marian van Noord en Jianet de Vries wezen mij de weg door Dijkzigt, organiseerden de eindeloze stapels paperassen en wisten immer een plek te vinden in de agenda van Jan IJzermans.

Dankzij dr. R.L. Marquet vond ik niet alleen een onderwerp maar ook een plek op het Laboratorium voor Chirurgie. Beste Richard, de ontspannen maar prikkelende sfeer op het lab is jouw verdienste. Jouw vermogen om altijd een andere invalshoek te vinden heeft menig idee opgeleverd. Aan alle andere collega's met wie ik langere- of korte- tijd kamer, lab of koffiekamer heb gedeeld: de compjoeter problemen en dead-lines schepten een band; de onbedaarlijke pret, ongein en vriendschap zullen mij altijd bijblijven.

En tenslotte George Akkersdijk en Willem Marsman, de paranimfen. In navolging van jullie ging ik nadenken over een afstudeer onderwerp. Het doet me goed dat jullie er bij de finale ook bij zullen zijn. 


\section{Curriculum vitae auctoris}

Joos Heisterkamp werd geboren te Nijmegen op 6 februari 1972. Hij volgde het middelbaar onderwijs aan de Stedelijke Scholen Gemeenschap Nijmegen en de Kees Boeke School te Bilthoven, alwaar hij in 1990 het eindexamen VWO behaalde. Datzelfde jaar werd de studie Geneeskunde aan de Erasmus Universiteit Rotterdam begonnen.

In 1995 raakte hij in het kader van het afstudeeronderzoek voor het doctoraal examen betrokken bij het onderzoeksprogramma Laser treatment of experimental liver metastases. Onder begeleiding van dr. R. van Hillegersberg en dr. J.N.M. IJzermans werd de basis voor dit proefschrift gelegd.

$\mathrm{Na}$ het doctoraal examen, augustus 1996, werd de studie onderbroken om als wetenschappelijk medewerker verbonden van de afdeling Algemene Heelkunde, Academisch Ziekenhuis Rotterdam (hoofd prof. dr. J. Jeekel) het onderzoek voort te zetten. Middels een Revolving Fund van het Academisch Ziekenhuis Rotterdam werd in samenwerking met de afdeling Radiologie (hoofd prof dr. J.S. Laméris, later prof. dr. G.P. Krestin) de mogelijkheid van MR-guided laser treatment for liver tumours verder geëxploreerd.

In augustus 1998 is de studie Geneeskunde hervat en volgt hij de co-assistentschappen in de postdoctorale fase aan de Erasmus Universiteit Rotterdam. 
